Athens University of Economics and Business DEPARTMENT OF INFORMATICS

Graduate Program in Computer Science

Scheduling in Computer and Communication Systems and

Generalized Graph Coloring Problems

Ph.D. Thesis

by

Giorgio Lucarelli

Athens, October 2009 



\title{
Scheduling in Computer and Communication Systems and \\ Generalized Graph Coloring Problems
}

\author{
Ph.D. Thesis by Giorgio Lucarelli
}

Advisory committee: Ioannis Milis (Supervisor)

Evangelos Magirou

Martha Sideri

Approved on the 2nd October 2009 by

Elias Koutsoupias, Professor

Department of Informatics and Telecommunications

National and Kapodistrian University of Athens

Evangelos Magirou, Professor

Department of Informatics

Athens University of Economics and Business

Ioannis Milis, Associate Professor

Department of Informatics

Athens University of Economics and Business

Vangelis Th. Paschos, Professor

LAMSADE

Université Paris-Dauphine

Martha Sideri, Associate Professor

Department of Informatics,

Athens University of Economics and Business

Stathis Zachos, Professor

School of Electrical and Computer Engineering

National Technical University of Athens

Vassilis Zissimopoulos, Professor

Department of Informatics and Telecommunications

National and Kapodistrian University of Athens 



\section{Acknowledgements}

Before beginning, I would like to thank my supervisor Ioannis Milis for his support and mentoring over these years. I would also like to thank the members of my advisory committee, Evangelos Magirou and Martha Sideri, as well as, the members of the evaluation committee, Elias Koutsoupias, Vangelis Th. Paschos, Stathis Zachos and Vassilis Zissimopoulos, for their observations and comments.

During my doctorate, I worked with Evripidis Bampis, Nicolas Bourgeois, Alexander Kononov, Ioannis Milis and Vangelis Th. Paschos. I want to thank them all for the collaboration.

My graduate studies were funded by the project PENED 2003. The project is cofinanced $75 \%$ of public expenditure through EC-European Social Fund, 25\% of public expenditure through Ministry of Development-General Secretariat of Research and Technology of Greece and through private sector, under measure 8.3 of Operational Programme "Competitiveness" in the 3rd Community Support Programme.

Many thanks to my friends Danae, Michael and Pantelis for going out and having a great time. Finally, special thanks to Katerina for her patience and support for more than ten years. 



\section{Contents}

Contents

$\begin{array}{lll}\text { Summary } & \text { iii }\end{array}$

$\Pi \varepsilon \rho i \lambda \eta \psi \eta \quad$ v

1 Introduction $\quad 1$

1.1 Generalized graph coloring problems . . . . . . . . . . . . . . 1

1.2 Motivation: Scheduling in computer and communication systems . . 2

1.3 Organization of the thesis $\ldots \ldots \ldots \ldots$. . . . . . . 4

2 Related work $\quad 7$

2.1 Vertex/Edge-Coloring . . . . . . . . . . . . . . . 7

2.2 Bounded Vertex/Edge-Coloring . . . . . . . . . . . . . . . . . 8

2.3 Max-Vertex/Edge-Coloring . . . . . . . . . . . . . . . . . 10

2.4 Bounded Max-Vertex/Edge-Coloring . . . . . . . . . . . . . . . 12

3 Preliminaries and Notation $\quad 13$

3.1 Preliminaries . . . . . . . . . . . . . . . . . . . 13

3.2 Notation . . . . . . . . . . . . . . . . . . . 16

4 Max-Edge-Coloring on general and bipartite graphs $\quad 17$

4.1 Preliminaries . . . . . . . . . . . . . . . . 17

$4.2 f(\Delta)$-approximation algorithms for bipartite graphs $\ldots \ldots \ldots$

4.3 An 1.74-approximation algorithm for bipartite graphs . . . . . . . 29

4.4 Bi-valued graphs . . . . . . . . . . . . . . . . . . 32

5 Max-Edge-Coloring on trees $\quad 35$

5.1 Stars of chains . . . . . . . . . . . . . . . 35

5.2 A $3 / 2$ approximation algorithm . . . . . . . . . . . . 37

5.3 Moderately exponential approximation algorithms . . . . . . . . . . . . . 40

5.4 Polynomial Time Approximation Scheme . . . . . . . . . . . . . 46 
6 Bounded Max-Edge-Coloring $\quad 49$

6.1 General and bipartite graphs . . . . . . . . . . . . . . . . . 49

6.2 NP-completeness for trees . . . . . . . . . . . . . . . . 53

6.3 A 2-approximation algorithm for trees . . . . . . . . . . 55

7 Bounded Max-Vertex-Coloring $\quad 57$

7.1 A simple split algorithm . . . . . . . . . . . . . . . 57

7.2 A generic scheme . . . . . . . . . . . . . . 58

8 Conclusions and open questions $\quad 61$

$\begin{array}{ll}\text { Bibliography } & 65\end{array}$ 


\section{Summary}

The thesis deals with weighted generalizations of the classical graph vertex/edgecoloring problems motivated by scheduling arising in computer and communication systems.

The most general problems we attack are called bounded max-vertex/edgecoloring problems and take as input a vertex/edge weighted graph and a bound $b$. In these problems each color class is of cardinality at most $b$ and of weight equal to that of the heaviest vertex/edge in this class. The objective is to find a proper coloring of the input graph minimizing the sum of all color classes' weights. For unit weights these problems reduce to the known bounded coloring problems, while in the absence of the cardinality bound we get the (unbounded) max-coloring problems.

The max-coloring problems have been well motivated and studied in the literature. Max-vertex-coloring problems arise in the management of dedicated memories, organized as buffer pools, which is the case for wireless protocol stacks like GPRS or 3G. Max-edge-coloring problems arise in switch based communication systems, like SS/TDMA, where messages are to be transmitted through direct connections established by an underlying network. Moreover, max-coloring problems correspond to scheduling jobs with conflicts in multiprocessor or batch scheduling environments. However, in all practical applications there exist physical constraints on the number of entities (corresponding to vertices/edges of a graph) assigned the same resource (color), which motivate the study of the bounded max-coloring problems.

In the first part of the thesis we present new complexity and approximation results for several variants of the max-edge-coloring problem with respect to the class of the underlying graph. In particular, we present polynomial algorithms for special graph classes (bounded degree trees, stars of chains) and approximation results for NP-complete variants. For bipartite graphs we present a series of four approximation algorithms; the last of them achieves an 1.74 approximation ratio and improves substantially the known ratio of 2 . For trees we give a $3 / 2$-approximation algorithm, two parameterized approximation algorithms and finally a PTAS. We also prove that the problem is NP-complete for complete bi-valued graphs and we present an asymptotic 4/3-approximation algorithm for general bi-valued graphs.

In the second part of the thesis we give the first known results for the bounded max-coloring problems. For the bounded max-edge-coloring problem, we prove approximation factors of at most 3 for general and bipartite graphs and 2 for trees. Furthermore, we show that this bounded problem is NP-complete for trees. This is the first complexity result for any max-coloring problem on trees. For the bounded 
max-vertex-coloring problem we present a generic scheme which becomes a 17/11approximation algorithm for bipartite graphs, a PTAS for bipartite graphs when $b$ is fixed and also a PTAS for trees even if $b$ is part of the problem's instance. 


\section{$\Pi \varepsilon \rho i \lambda \eta \psi \eta$}

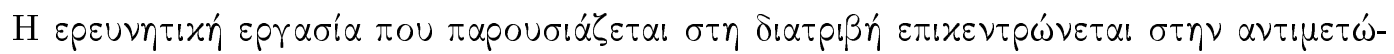

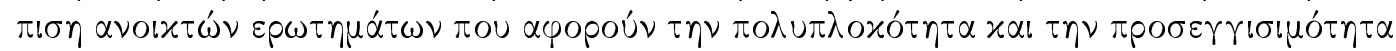

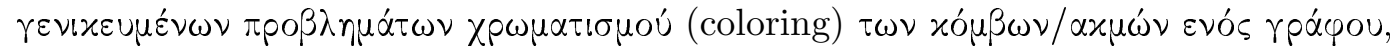

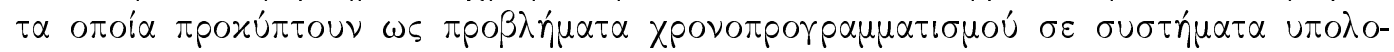

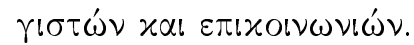

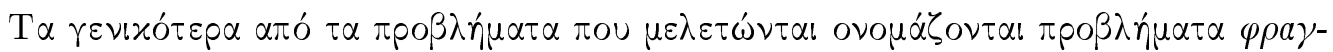

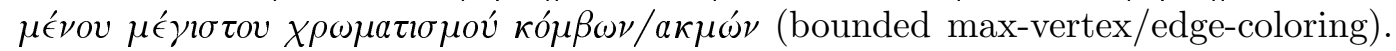

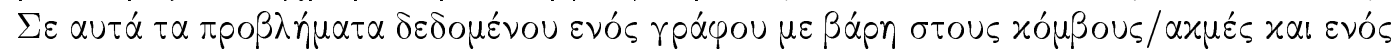

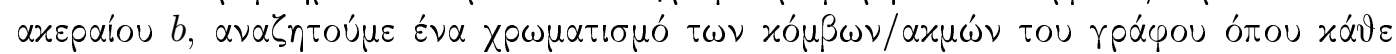

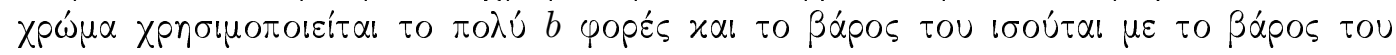

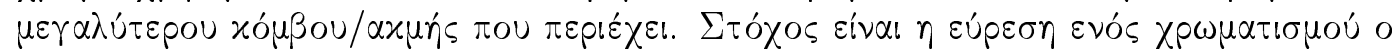

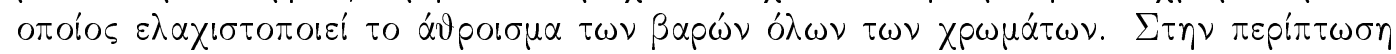

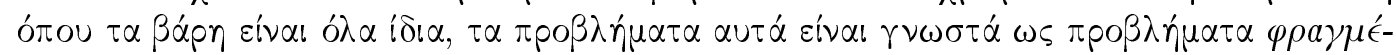

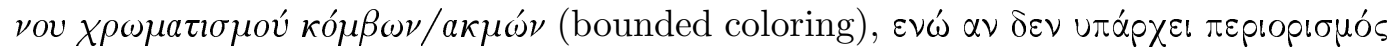

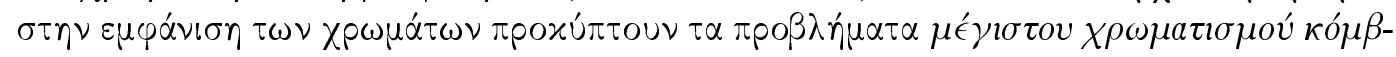
$\omega \nu / a \kappa \mu \omega ́ \nu$ (max-coloring).

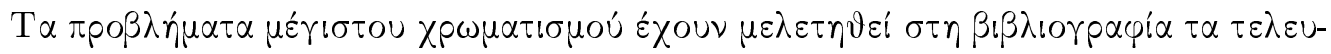

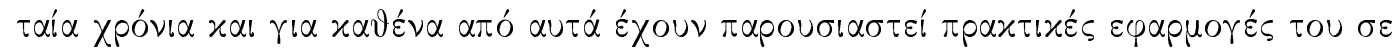

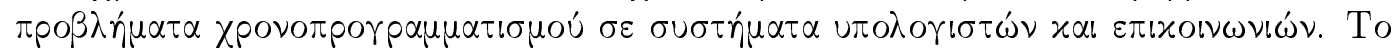

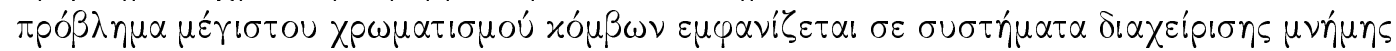

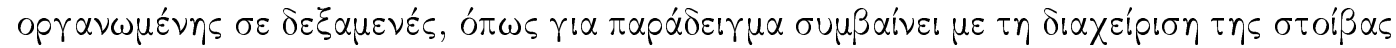

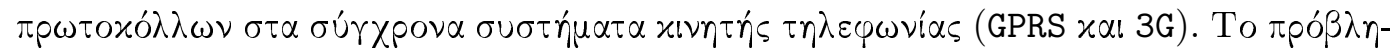

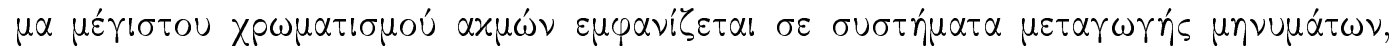

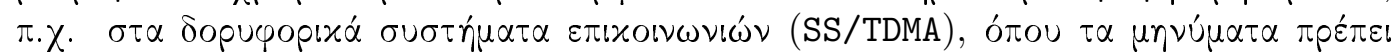

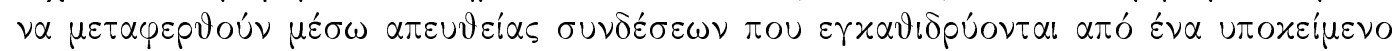

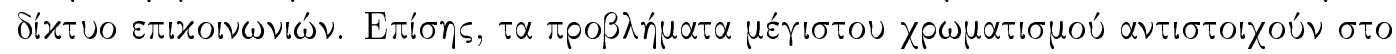

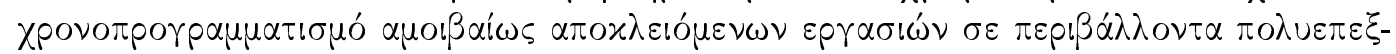

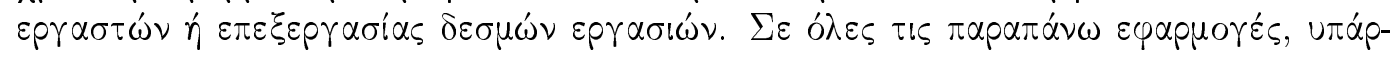

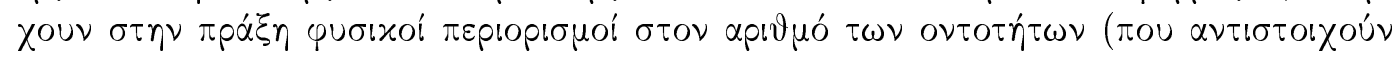

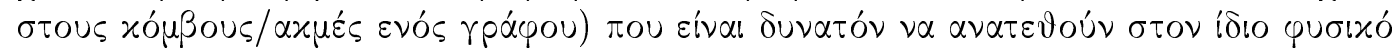

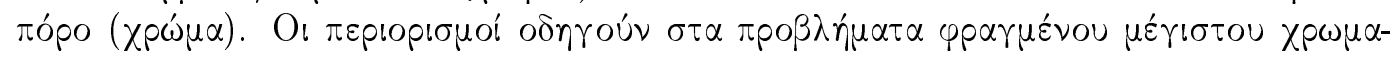

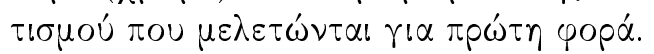

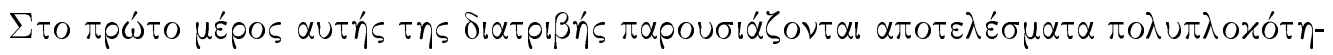

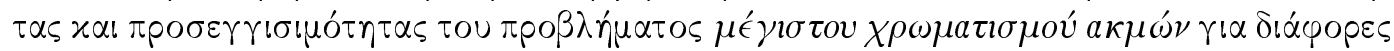

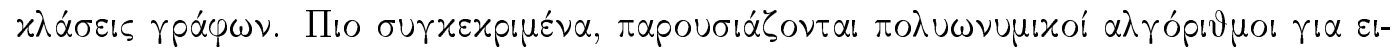




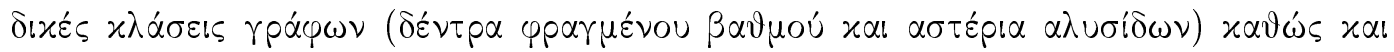

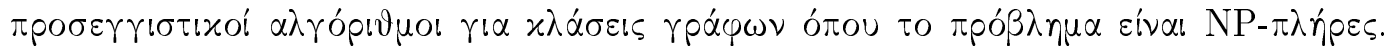

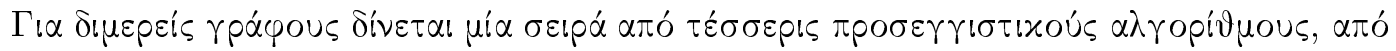

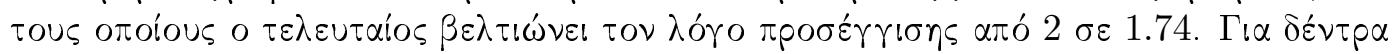

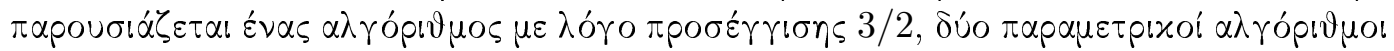

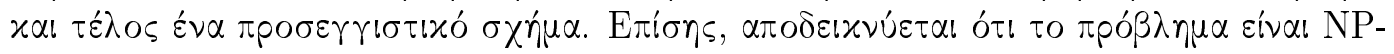

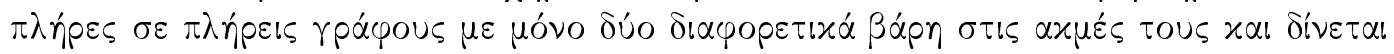

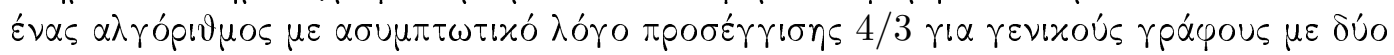
Bápr.

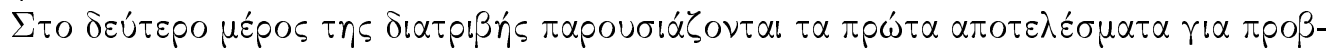

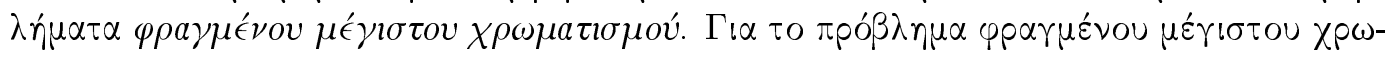

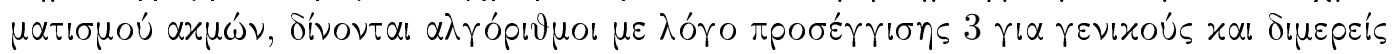

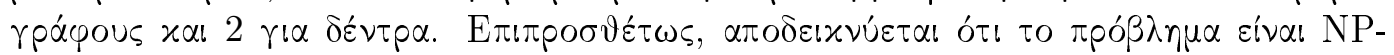

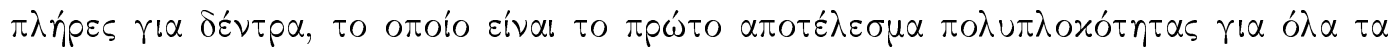

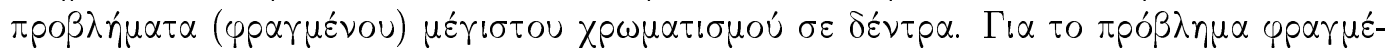

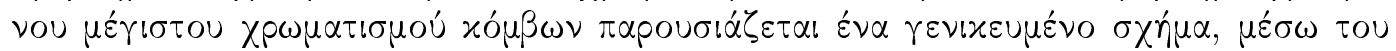

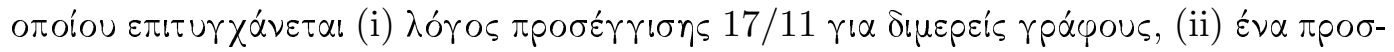

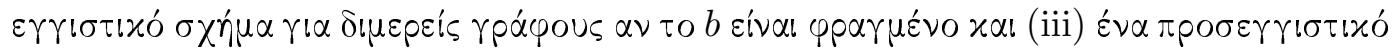

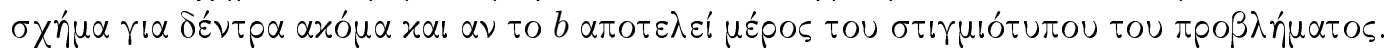




\section{Chapter 1}

\section{Introduction}

In this first chapter of the thesis, we introduce weighted generalizations of the classical vertex and edge coloring problems, which correspond to scheduling problems arising in computer and communication systems. We also give an outline of the next chapters of the thesis and a preview of our results.

\subsection{Generalized graph coloring problems}

A vertex- (resp. edge-) coloring of a graph $G=(V, E)$ is a partition $\mathcal{C}=\left\{C_{1}, C_{2}\right.$, $\left.\ldots, C_{k}\right\}$ of its vertex (resp. edge) set into color classes such that each class $C_{i}$ constitutes an independent set (resp. matching) of $G$. Such a coloring is called a proper coloring of $G$. The classical vertex- (resp. edge-) coloring problem asks for a proper coloring of a graph $G$ such that the number, $k$, of colors is minimized. This minimum number of colors required to color the vertices (resp. edges) of a graph $G$ is also known as the chromatic number $\chi(G)$ (resp. chromatic index $\chi^{\prime}(G)$ ) of $G$.

In the bounded vertex-coloring [4] (resp. bounded edge-coloring [2]) problem we are, in addition, given a positive integer $b$ and we ask for a proper coloring where each color appears at most $b$ times, i.e., $\left|C_{i}\right| \leq b, 1 \leq i \leq k$. The goal in both bounded coloring problems is also to minimize the number, $k$, of colors.

In several application domains the following weighted generalizations of graph coloring problems arise: Each vertex $u$ (resp. edge $e$ ) of $G$ is associated with a positive integer weight $w(u)$ (resp. $w(e)$ ) and we ask again for a partition $\mathcal{C}=$ $\left\{C_{1}, C_{2}, \ldots, C_{k}\right\}$ of the vertex (resp. edge) set of $G$ into color classes, each one of weight $w_{i}=\max \left\{w(v) \mid v \in C_{i}\right\},\left(\right.$ resp. $\left.w_{i}=\max \left\{w(e) \mid e \in C_{i}\right\}\right)$, such that the total weight of the partition $W=\sum_{i=1}^{k} w_{i}$ is minimized. These coloring problems are known as max-(vertex-)coloring [52] and max-edge-coloring.

The presence of a bound $b$ to the cardinality of each color leads to the bounded max-vertex-coloring and bounded max-edge-coloring problems, respectively.

In this thesis we shall denote the above coloring problems as follows:

- VC/EC: vertex/edge-coloring problem 
- $\mathrm{VC}(b) / \mathrm{EC}(b)$ : bounded vertex/edge-coloring problem

- $\operatorname{VC}(w) / \mathrm{EC}(w)$ : max-vertex/edge-coloring problem

- $\mathrm{VC}(w, b) / \mathrm{EC}(w, b)$ : bounded max-vertex/edge-coloring problem

where $\mathrm{VC}$ and $\mathrm{EC}$ are used to denote the vertex and edge versions of the classical coloring problem, respectively, $b$ indicates the existence of a cardinality bound to colors, and $w$ shows the existence of weights on the vertices or the edges of the input graph.

Clearly, both $\mathrm{VC}(b)$ and $\mathrm{VC}(w)$ reduce to the classical VC problem, if $b=|V|$ and $w(u)=1, \forall u \in V$, respectively. Moreover, $\operatorname{VC}(w, b)$ reduces to all $\mathrm{VC}, \mathrm{VC}(b)$ and $\operatorname{VC}(w)$ problems. Analogous reductions exist between the $\mathrm{EC}, \mathrm{EC}(b), \operatorname{EC}(w)$ and $\operatorname{EC}(w, b)$ problems. These reductions are shown in Figure 1.1, where each directed edge indicates that the source problem reduces to the destination one.
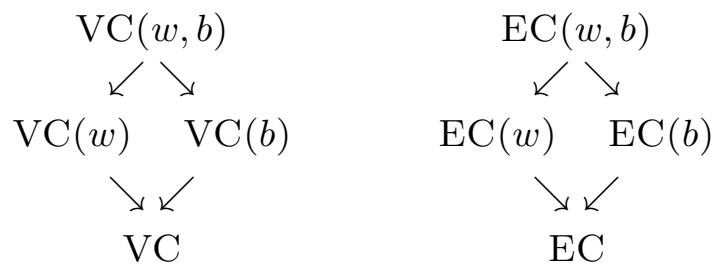

Figure 1.1: Reductions between coloring problems.

Remark that any generalization of the edge-coloring problem on a general graph $G$ is equivalent to the corresponding vertex-coloring problem on the line graph, $L(G)$, of $G$. Thus, the results for any vertex-coloring problem on a graph $G$ apply also to the corresponding edge-coloring one on the graph $L(G)$ and vice versa, if both $G$ and $L(G)$ are in the same graph class. Note, however, that this is true for general graphs and chains, but not for the most other special graph classes, including bipartite graphs and tree, since they are not closed under line graph transformation (e.g., the line graph of a bipartite graph is not anymore a bipartite one).

\subsection{Motivation: Scheduling in computer and communi- cation systems}

In this section we motivate both vertex and edge variants of our coloring problems as scheduling problems arising in computer and communication systems.

In many applications with strict memory constraints a dedicated memory allocation manager is often used in order to optimize the memory utilization and the performance of the system. For example, in wireless communications [51, 52], where mobile devices have to manage a protocol stack (e.g., GPRS or 3G) with stringent memory requirements, a dedicated memory manager is the natural choice. A common approach in the design of such a memory manager is the use of an amount of 
the total memory as a segregated buffer pool which consists of a set of buffers of different sizes. As a memory request arrives it is scheduled to a free buffer of large enough size. Memory requests in different time intervals can use the same buffer which, however, should be of size greater than the largest request allocated to this buffer. The aim of such a memory manager is to minimize the total size of the buffers used to service a set of requests and thus the amount of the total memory which is used as a segregated buffer pool.

The above problem correspond directly to the $\mathrm{VC}(w)$ problem, with memory requests corresponding to the set of vertices of a graph $G$ and their weights to the size of the memory requests. An edge between two vertices of $G$ exists if the corresponding requests have to be served in overlapping time intervals. So, a partition of $G$ into independent sets corresponds to an allocation of the requests to buffers, with each set corresponding to a set of requests that can be allocated to the same buffer. The weight of a set corresponds to the buffer size which should be at least equal to the largest memory request in this set. Thus, the total size of the buffers needed to service a given set of requests is equal to the total weight of the partition of $G$.

On the other hand, the $\mathrm{EC}(w)$ problem arises in single hop communication systems, like SS/TDMA [35, 46], where messages are to be transmitted directly from senders to receivers through connections established by an underlying switching network. Any node of such a system cannot participate in more than one transmissions at the same time, while messages between different pairs of senders and receivers can be transmitted simultaneously. The scheduler of such a system establishes successive configurations of the switching network, each one routing a non-conflicting subset of the messages from senders to receivers. Given the transmission time of each message, the transmission time of each configuration equals to the longest message transmitted. Moreover, in practice, there is a non negligible setup delay to establish each configuration. The aim is to find a sequence of configurations such that all the messages are transmitted and the total transmission time (including setup delays) is minimized.

It is easy to see that the above situation corresponds directly to the $\operatorname{EC}(w)$ problem: senders and/or receivers correspond to the vertices of the graph $G$, (transmission times of) messages correspond to (weights of) edges of $G$ and configurations correspond to colors. Although the graph $G$ obtained is originally a weighted directed multi-graph it can be considered as an undirected one, since the directions of its edges do not play any role in the objective function we study here.

The presence of the setup delay in the instance of the $\mathrm{EC}(w)$ problem, can be easily handled: by adding $d$ to the weight of all edges of $G$, the weight of each color class will be also increased by $d$, incorporating its set up delay. Furthermore, a standard idea to decrease the completion time of a schedule is to allow preemption $[1,17,35]$, i.e., interrupt the service of a (set of) scheduled activity(ies) and complete it (them) latter. It is obvious that allowing preemption in the $\mathrm{EC}(w)$ problem will result in increasing the number of colors in a solution. In this case, the presence of a set up delay $d$ plays a crucial role in the hardness of the (preemptive) $\mathrm{EC}(w)$ problem. 
In all applications mentioned above, context-related entities require their service by physical resources for a time interval. However, there exists in practice a natural constraint on the number of entities assigned the same resource or different resources at the same time. Indeed, the number of memory requests assigned the same buffer is determined by strict deadlines on their completion times, while the number of messages assigned at the same time to different channels is bounded by the number of the available resources. The existence of such a constraint motivates the bounded max-coloring problems $\mathrm{VC}(w, b)$ and $\operatorname{EC}(w, b)$.

The $\operatorname{VC}(b)$ and $\operatorname{VC}(w, b)$ problems are also equivalent with scheduling problems where jobs correspond to the vertices of a graph $G=(V, E),|V|=n$, which describes incompatibilities between them. If all jobs have unit processing times, then the $\mathrm{VC}(b)$ problem is equivalent to the $\mathrm{Pb} \mid$ graph,$p_{j}=1 \mid C_{\max }$ scheduling problem, where the goal is to minimize the makespan of the schedule of $n$ jobs on $b$ processors under the constraint that adjacent jobs cannot be scheduled at the same time. This problem is also known as Mutual Exclusion Scheduling (MES) [4]. If jobs have different processing times, then the $\operatorname{VC}(w, b)$ problem is equivalent to the $1 \mid p-$ batch, graph, $b<n \mid C_{\max }$ parallel batch scheduling problem, where the goal is to minimize the makespan of the batch schedule of $n$ jobs on one machine under the constraints that adjacent jobs cannot be scheduled at the same batch and each batch is of cardinality at most $b[22,26]$.

Analogous scheduling definitions can be given for both the $\operatorname{EC}(b)$ and $\operatorname{EC}(w, b)$ problems. For these problems the jobs correspond to the edges of the graph, while adjacent jobs cannot be scheduled at the same time.

\subsection{Organization of the thesis}

In this thesis we present complexity and approximation results for the coloring problems introduced in the previous section, which correspond to scheduling problems arising in computer and communication systems.

In Chapter 2, we review the known results for the coloring problems, with respect to the class of the underlying graph.

In Chapter 3, we exploit the relation between our coloring problems and two other well studied problems, and we give two preliminary results. First we use a relation with the list coloring problem and we obtain that all $\mathrm{VC}(w), \operatorname{VC}(w, b)$, $\operatorname{EC}(w)$ and $\operatorname{EC}(w, b)$ problems are polynomial on trees, if the number, $k$, of colors is fixed. Next, through a transformation to the set cover problem we show an $H_{b^{-}}$ approximation algorithm for all $\mathrm{VC}(b), \mathrm{VC}(w, b), \mathrm{EC}(b), \mathrm{EC}(w, b)$ problems on general graphs, if the cardinality bound, $b$, is fixed. Furthermore, we present two more observations on the approximability of our coloring problems. The first shows the existence of an $(e \cdot \rho)$-approximation algorithm for any max-coloring problem on a hereditary class of graphs, where $\rho$ is a known approximation ratio for the corresponding unweighted coloring problem on the same class. The second observation shows a $b / 2$ approximation ratio for all the bounded coloring problems. We close this chapter with the notation that we shall use in this thesis. 
In Chapter 4, we deal with the $\mathrm{EC}(w)$ problem on general, bipartite and bivalued graphs. We first improve the analysis of a well known 2-approximation algorithm presented in [46], and using this analysis we derive even better approximation ratios for general and bipartite graphs. Next, we present four approximation algorithms for the $\mathrm{EC}(w)$ problem on bipartite graphs, each one improving the previous ones for some values of the maximum degree of the input graph. The first three algorithms achieve approximation ratios increasing with the maximum degree of the graph, while the forth one improves the known 2 approximation ratio to 1.74 for bipartite graphs. Finally, we prove that the $\mathrm{EC}(w)$ problem is NP-complete for complete bi-valued graphs and we present an asymptotic $4 / 3$-approximation algorithm for general bi-valued graphs.

In Chapter 5, we present results for the $\mathrm{EC}(w)$ problem on trees. We first show that the $\operatorname{EC}(w)$ problem is polynomial for stars of chains (also known as spiders). Next, we present a 2-approximation algorithm for trees. Combining this algorithm and our analysis of the known 2-approximation one [46], we obtain a $3 / 2$ approximation ratio for the $\operatorname{EC}(w)$ problem on trees. Next, we propose two moderately exponential approximation algorithms for trees that improve the $3 / 2$ ratio with running time much better than that needed for the computation of an optimal solution. More interestingly, we have also finally succeeded to derive a PTAS for the $\operatorname{EC}(w)$ problem on trees.

In Chapter 6, we give complexity and approximation results for the $\mathrm{EC}(w, b)$ problem. We first prove lower and upper bounds to the number of colors of any solution for the $\mathrm{EC}(w, b)$ problem. Using these bounds, we present an approximation algorithm of ratios $3-\frac{2}{\sqrt{2 b}}$ and $3-\frac{2}{\sqrt{b}}$ for general and bipartite graphs, respectively. Furthermore, we show that the $\operatorname{EC}(w, b)$ problem is NP-complete for trees, which is the first complexity result for any max-coloring problems on trees. For this last problem we also give a 2-approximation algorithm.

In Chapter 7, we present a 2-approximation algorithm for the $\mathrm{VC}(w, b)$ problem on bipartite graphs. This algorithm reduces to a $4 / 3$-approximation algorithm for the $\mathrm{VC}(b)$ problem on bipartite graphs, closing the approximability question for this problem. Then, we use this 2-approximation algorithm to obtain a generic scheme that leads to the following results for the $\mathrm{VC}(w, b)$ problem: (i) a 17/11approximation algorithm for bipartite graphs, (ii) a PTAS for bipartite graphs, when $b$ is fixed, and (iii) a PTAS for trees, even if $b$ is part of the problem's instance.

In Chapter 8 we summarize our results and we discuss questions that remain still open.

For the reader to have a chart preview of the problems we study and the current state of the art on their complexity and approximability, we include here Table 1.1, where our results (in bold) are summarized together with known ones that are reviewed in the next chapter. 


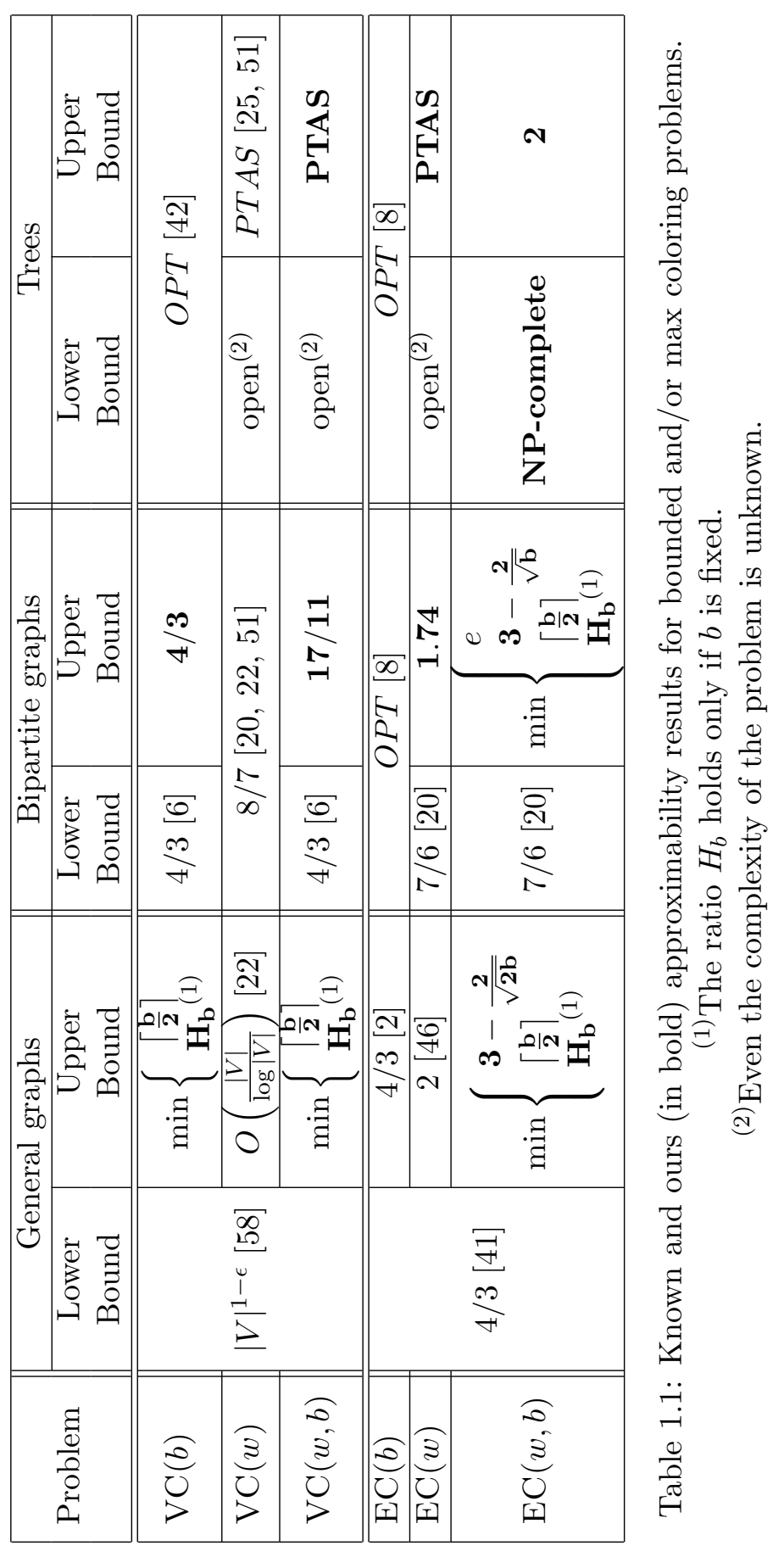




\section{Chapter 2}

\section{Related work}

In this chapter we review the known results for the coloring problems introduced in the previous chapter, with respect to the class of the underlying graph. Although our results concern general graphs, bipartite graphs and trees, for the sake of completeness we present here results for several other interesting classes of graphs.

\section{$2.1 \quad$ Vertex/Edge-Coloring}

The vertex coloring (or chromatic number) problem on general graphs is one of Karp's 21 NP-complete problems [45]. It is also known to be NP-complete on planar graphs even for three colors [32], although four colors suffice to color any planar graph (see for example, [54]), as well as on circular arc graphs [31]. On the other hand, the $\mathrm{VC}$ problem can be solved in polynomial time for several other classes of graphs, including perfect graphs [37], chordal and interval graphs [33], split graphs, comparability graphs and cographs [34]. For bipartite graphs, trees, cliques and chains the $\mathrm{VC}$ problem is trivially polynomial.

In [58] it is shown that it is NP-hard to approximate the VC problem on general graphs within a factor of $|V|^{1-\epsilon}$, for all $\epsilon>0$, while an algorithm of approximation ratio $O\left(|V| \frac{(\log \log |V|)^{2}}{(\log |V|)^{3}}\right)$ has been presented in [38]. For planar graphs, the approximability question for the VC problem is closed, since the NP-completeness proof [32] and the four color theorem [54] lead to a 4/3 inapproximability result and approximation algorithm, respectively. Moreover, a 3/2-approximation algorithm has been proposed in [44] for the VC problem on circular arc graphs.

For the edge coloring (or chromatic index) problem, it is well known that its optimal solution consists of either $\Delta$ or $\Delta+1$ colors [56]. However, it is NP-hard to decide between these two values even on cubic graphs [41] and on comparability graphs [11] (and hence on perfect graphs). The first result implies also a $4 / 3$ inapproximability bound for the problem. On the other hand, the EC problem is solvable in polynomial time on bipartite graphs [47] (and hence on trees), and cliques [27].

There are many classes of graphs for which the VC problem is polynomial, while 
the complexity of the EC problem still remains open. Examples of such classes are chordal, split and interval graphs and cographs. Moreover, the complexity of the EC problem is open for planar graphs, while partial results are known for split [12], interval [7] and planar graphs [55, 57].

As mentioned above, any graph has a $(\Delta+1)$-edge-coloring and this coloring can be found in $O(\min \{|V| \Delta \log |V|,|E| \sqrt{|V| \log |V|}\})$ time [29]. Using such a $(\Delta+1)$ edge-coloring, a 4/3-approximation algorithm is obtained for the EC problem on general graphs of $\Delta \geq 3$; recall that the EC problem is trivially polynomial for graphs of maximum degree two.

Table 2.1 summarizes known complexity results for the classical coloring problems on several well-studied strong classes. Results for more specific classes of graphs can be found in $[43,49]$.

\begin{tabular}{|c|c|c|}
\hline Graph & Vertex-Coloring & Edge-Coloring \\
\hline general & $\begin{array}{l}\text { NP-complete [45] } \\
\omega \leq \chi \leq \Delta+1\end{array}$ & $\begin{array}{l}\text { NP-complete }[41] \\
\chi^{\prime}=\Delta \text { or } \Delta+1[56]\end{array}$ \\
\hline $\begin{array}{l}\text { perfect } \\
\text { chordal }\end{array}$ & $\begin{array}{l}\chi=\omega[37] \\
\chi=\omega[33]\end{array}$ & $\begin{array}{l}\text { NP-complete } \\
\text { open }\end{array}$ \\
\hline split & $\chi=\omega[34]$ & $\chi^{\prime}= \begin{cases}\Delta+1, & \text { if } \Delta \text { is odd [12] } \\
\text { open, } & \text { if } \Delta \text { is even }\end{cases}$ \\
\hline comparability & $\chi=\omega[34]$ & NP-complete [11] \\
\hline bipartite & $\chi=\omega=2$ & $\chi^{\prime}=\Delta[47]$ \\
\hline trees & $\chi=\omega=2$ & $\chi^{\prime}=\Delta$ \\
\hline cograph & $\chi=\omega[34]$ & open \\
\hline chains & $\chi=\omega=2$ & $\chi^{\prime}=\Delta=2$ \\
\hline circular arc & NP-complete [31] & \\
\hline interval & $\chi=\omega[33]$ & $\chi^{\prime}= \begin{cases}\Delta, & \text { if } \Delta \text { is odd }[7] \\
\text { open, } & \text { if } \Delta \text { is even }\end{cases}$ \\
\hline clique & $\chi=\omega=|V|$ & $\chi^{\prime}=\left\{\begin{array}{ll}\Delta, & \text { if } \Delta \text { is odd } \\
\Delta+1, & \text { if } \Delta \text { is even }\end{array}[27]\right.$ \\
\hline planar & $\begin{array}{l}\text { NP-complete }[32] \\
\chi \leq 4[54]\end{array}$ & $\chi^{\prime}= \begin{cases}\Delta, & \text { for } \Delta \geq 7[55,57] \\
\text { open, } & \text { otherwise }\end{cases}$ \\
\hline
\end{tabular}

Table 2.1: Complexity results for the classical coloring problems.

\subsection{Bounded Vertex/Edge-Coloring}

The bounded vertex-coloring (or Mutual Exclusion Scheduling [4]) problem is NP-complete for general, circular arc and planar graphs, as a generalization of the $\mathrm{VC}$ problem. The complexity of the $\mathrm{VC}(b)$ problem has been also extensively studied on special graph classes. It is NP-complete for cographs [6], interval graphs [6] (and hence for chordal and perfect graphs) and bipartite graphs even for three 
colors [6]. This last result implies also a $4 / 3$ inapproximability bound for the $\mathrm{VC}(b)$ problem on bipartite graphs as well as the NP-completeness of the $\mathrm{VC}(b)$ problem on comparability graphs. On the other hand, the $\mathrm{VC}(b)$ problem is polynomial for split graphs [6], trees [42] (and hence for chains) and cliques [19]. Note, finally, that for the number of colors, $k^{*}$, in an optimal solution of the $\mathrm{VC}(b)$ problem it holds that $\max \left\{\left\lceil\frac{|V|}{b}\right\rceil, \chi\right\} \leq k^{*} \leq \chi+\left\lfloor\frac{|V|-\chi}{b}\right\rfloor[40]$.

Table 2.2 summarizes known complexity results for the $\mathrm{VC}(b)$ problem. For further results the readers referred to [30] and the references therein.

\begin{tabular}{|l|l|}
\hline Graph & Complexity \\
\hline \hline general & NP-complete ${ }^{(1)}$ \\
\hline perfect & NP-complete \\
chordal & NP-complete \\
split & polynomial $[6]$ \\
comparability & NP-complete \\
bipartite & NP-complete $[6]$ \\
trees & polynomial $[42]$ \\
cograph & NP-complete $[6]$ \\
chains & polynomial \\
\hline $\begin{array}{l}\text { circular arc } \\
\text { interval }\end{array}$ & NP-complete \\
clique & NP-complete $[6]$ \\
\hline planar & polynomial $[19]$ \\
\hline
\end{tabular}

Table 2.2: Complexity results for the bounded vertex-coloring problem. ${ }^{(1)}$ The NPcompleteness comes from the VC problem.

The complexity of the bounded edge-coloring problem is related substantially to the complexity of the EC problem by the following proposition.

Proposition 1 (de Werra [18]). For any $k \geq \Delta$, a bipartite multigraph has a decomposition into $k$ colors such that for all $i$ and $j, 1 \leq i, j \leq k$, it holds that ||$C_{i}|-| C_{j}|| \leq 1$.

The proof of this proposition is based on the fact that the graph which is induced by the edges of any two colors, $C_{i}$ and $C_{j}$, is a collection of chains and even cycles. Without loss of generality, assume that $\left|C_{i}\right|-\left|C_{j}\right|>2$. Thus, there is at least one subchain in $C_{i} \cup C_{j}$ whose the number of edges from $C_{i}$ is greater by one than the number of edges from $C_{j}$. A swap of the edges of $C_{i}$ and $C_{j}$ of such a subchain decreases by one the cardinality of $C_{i}$ and increases by one the cardinality of $C_{j}$. Therefore, for any $k \geq \Delta$ we can create by successive swaps a solution of $k$ colors such that ||$C_{i}|-| C_{j}|| \leq 1$.

Clearly, if we replace the condition $k \geq \Delta$ by $k \geq \chi^{\prime}$ then Proposition 1 holds also for general graphs. Note also that an optimal solution for the $\mathrm{EC}(b)$ problem 
consists of at least $\left[\frac{|E|}{b}\right]$ colors. Thus, if $\left[\frac{|E|]}{b}\right]>\Delta$ then an optimal solution for the $\mathrm{EC}(b)$ problem on general graphs can be found using Proposition 1. Otherwise, the $\mathrm{EC}(b)$ problem is as hard as the EC problem.

Concluding, the $\mathrm{EC}(b)$ problem is $4 / 3$-inapproximable on general graphs, while a 4/3-approximation algorithm is obtained by Vizing's theorem [56] and the discussion above. On the other hand, the $\mathrm{EC}(b)$ problem is polynomial on bipartite graphs by Proposition 1, a result that has been also proved independently in [8] in matrix decomposition context. For complexity results for other classes of graphs see the edge-coloring column of Table 2.2, as well as [43, 49].

Finally, Alon in [2] proved that the $\mathrm{EC}(b)$ problem is polynomially solvable on general graphs if the cardinality bound $b$ is fixed. To prove this, he based on the fact that "if $\chi^{\prime}=\Delta+1$ then $|E| \geq \frac{1}{8}\left(3 \Delta^{2}+6 \Delta-1\right)$ " [28].

\subsection{Max-Vertex/Edge-Coloring}

The max-vertex-coloring problem is strongly NP-hard even for (i) bipartite graphs and edge weights $w(e) \in\{1,2,3\}[22,51]$ (and hence for comparability and perfect graphs), (ii) split graphs and edge weights $w(e) \in\{1,2\}[22]$ (and hence for chordal graphs), (iii) planar bipartite graphs [20], and (iv) interval graphs [25, 52] (and hence for circular arc graphs).

Moreover, it has been shown that the $\mathrm{VC}(w)$ problem on bipartite graphs with edge weights $w(e) \in\{1,2,3\}[22,51]$ and planar bipartite graphs [20] cannot be approximated within a ratio less than $8 / 7$. This bound has been attained for general bipartite graphs [20,51], while an $O(|V| / \log |V|)$-approximation algorithm for general graphs is known [22]. Although the complexity of the problem on trees is an open question, a PTAS for this case has been presented in $[25,51]$. In addition, a 4 approximation algorithm has been presented in [52] for perfect graphs; this ratio has been improved to $e$ in [24], using randomization/derandomization techniques. For $k$-colorable graphs, an approximation algorithm of ratio $\frac{k^{3}}{3 k^{2}-3 k+1}$ has been proposed in [25], leading to a 64/37-approximation algorithm for planar graphs. In addition, approximation algorithms of ratio 2 and 3 for interval and circular arc graphs, respectively, have been presented in [52], exploiting the relation between the $\mathrm{VC}(w)$ problem and online coloring. Furthermore, a PTAS for split graphs is known [20].

Moreover, the $\mathrm{VC}(w)$ problem is known to be polynomial on bipartite graphs and edge weights $w(e) \in\{1,2\}$ [22], cographs [22], and chains [25]. In fact, the algorithm for chains can be also extended for graphs of $\Delta=2$. Finally, a new algorithm for the $\operatorname{VC}(w)$ problem on chains which improves the complexity for this case from $O\left(|V|^{2}\right)$ to $O(|V| \cdot \log |V|)$ has been presented in [39].

Table 2.3 summarizes the above results for the $\mathrm{VC}(w)$ problem. Several results for even more restricted classes of graphs can be found in [20].

The max-edge-coloring problem is strongly NP-hard even for (i) complete balanced bipartite graphs [53], (ii) bipartite graphs of maximum degree three and edge weights $w(e) \in\{1,2,3\}$ [35, 46], (iii) cubic bipartite graphs [22], and (iv) 


\begin{tabular}{|l|c|c|}
\hline Graph & Lower Bound & Upper Bound \\
\hline \hline general & $|V|^{1-\epsilon^{(1)}}$ & $O\left(\frac{|V|}{\log |V|}\right)[22]$ \\
\hline \hline perfect & $8 / 7$ & $e[24]$ \\
\hline chordal & NP-complete & $e$ \\
\hline split & NP-complete $[22]$ & $P T A S[20]$ \\
\hline comparability & $8 / 7$ & $e$ \\
\hline bipartite & \multicolumn{2}{|c|}{$8 / 7[20,22,51]$} \\
\hline bipartite, $w \in\{a, b\}$ & \multicolumn{2}{|c|}{$O P T[22]$} \\
\hline trees $O P T[22]$ \\
\hline cograph & open $^{(2)}$ & $P T A S[25,51]$ \\
\hline chains & \multicolumn{2}{|c|}{$O P, 39]$} \\
\hline \hline circular arc & NP-complete & $3[52]$ \\
\hline interval & NP-complete $[25,52]$ & $2[52]$ \\
\hline clique & \multicolumn{2}{|c|}{$O P T$} \\
\hline \hline planar & $4 / 3^{(1)}$ & $64 / 37[25]$ \\
\hline
\end{tabular}

Table 2.3: Known approximability results for the max-vertex-coloring problem. ${ }^{(1)}$ This result comes from the VC problem. ${ }^{(2)}$ Even the complexity of the problem is unknown.

cubic planar bipartite graphs with edge weights $w(e) \in\{1,2,3\}$ [20]. Moreover, it has been shown that the $\mathrm{EC}(w)$ problem on $r$-regular bipartite graphs cannot be approximated within a ratio less than $\frac{2^{r}}{2^{r}-1}$, which for $r=3$ becomes $8 / 7$ [22]. This inapproximability result has been improved to $7 / 6$ for cubic planar bipartite graphs $[20]$.

On the other hand, a simple greedy 2-approximation algorithm has been presented in [46] for bipartite graphs (in fact, the same algorithm applies also for general graphs). In addition, a $\frac{2 \Delta-1}{3}$-approximation algorithm, for bipartite graphs of maximum degree $\Delta$, has been presented in [22], which gives an approximation ratio of $5 / 3$ for $\Delta=3$. A new algorithm for bipartite graphs with $\Delta=3$ has been presented in [20]; it achieves an approximation ratio of $7 / 6$ which attains, for this case, the known $7 / 6$ inapproximability bound. Finally, an algorithm that achieves approximation ratio $\rho_{\Delta}=\frac{\Delta}{\sum_{i=1}^{\Delta} \prod_{j=i}^{\Delta-1}\left(1-\frac{\rho_{j}}{\Delta}\right)}$, for graphs of maximum degree $\Delta$ has been proposed in [25]. This ratio is smaller than 2 only for bipartite graphs of maximum degree $\Delta \leq 7$.

Moreover, the $\mathrm{EC}(w)$ problem is known to be polynomial for a few very special cases including complete balanced bipartite graphs and edge weights $w(e) \in\{1,2\}$ [53], general bipartite graphs and edge weights $w(e) \in\{1,2\}$ [22], and chains [25]. In fact, the last result was presented for the $\mathrm{VC}(w)$ problem, and holds also for the $\mathrm{EC}(w)$ problem, since chains are closed under the linegraph transformation.

Finally, the preemptive-EC $(w)$ problem, without setup delays, for bipartite 
graphs is equivalent to the preemptive open shop scheduling problem which can be solved optimally in polynomial time [48]. However, with the presence of a setup delay, $d$, required to establish each color, the preemptive-EC problem on bipartite graphs becomes strongly NP-hard [35] and non approximable within a factor less than $7 / 6[17]$. Approximation algorithms for this problem of factors 2 and $2-\frac{1}{d+1}$ have been presented in [17] and [1], respectively.

\subsection{Bounded Max-Vertex/Edge-Coloring}

Clearly, any negative result for the $\mathrm{VC}(b) / \mathrm{VC}(w)$ and $\mathrm{EC}(b) / \mathrm{EC}(w)$ problems holds also for the $\operatorname{VC}(w, b)$ and $\mathrm{EC}(w, b)$ problems, respectively.

Known results for the $\mathrm{VC}(w, b)$ problem have appeared in the context of batch scheduling jobs with compatibilities (see e.g. [26]). In this problem the goal is to decompose the graph into a set of cliques (instead of colors/independent-sets). Thus, results for this problem on special graph classes lead to analogous results for the $\operatorname{VC}(w, b)$ problem on the complements of these classes. An interesting result that has been shown in this context is that the $\mathrm{VC}(w, b)$ problem is NP-complete for split graphs and $b=3[9]$, since the complement of a split graph remains in the same class.

Furthermore, a polynomial algorithm for general graphs and $b=2$ has been presented for the scheduling problem with compatibilities [10]. This algorithm can be used to obtain an analogous result for both $\operatorname{VC}(w, b)$ and $\operatorname{EC}(w, b)$ problems on general graphs, since general graphs are closed under complement and linegraph operations.

Finally, a 8/3-approximation algorithm is known for the preemptive-EC $(w, b)$ problem on bipartite graphs [15]. 


\section{Chapter 3}

\section{Preliminaries and Notation}

In this chapter we first relate our coloring problems with two well studied problems, namely list coloring and set cover. Using these relations we obtain preliminary results for the (bounded) max-coloring problems when either the number, $k$, of colors or the cardinality bound, $b$, are fixed.

Next, we present two preliminary approximation results for the bounded maxcoloring problems. The first one follows from a known general framework, which allows to convert a $\rho$-approximation algorithm for a coloring problem to an $e \cdot \rho$ approximation one for the corresponding max-coloring problem. The second approximation result follows by using a solution to a bounded coloring problem with cardinality bound $b=2$ to approximate a solution for an arbitrary bound $b$.

We close this chapter by the notation that we shall use throughout this thesis.

\subsection{Preliminaries}

\section{List coloring and fixed number of colors}

In several algorithms that we shall present in the next chapters, the following decision problem has to be answered (we present here the bounded vertex version of this problem; unbounded and/or edge versions are defined similarly):

FeAsible- $\mathrm{VC}(w, b)$

InstancE: A vertex weighted graph $G=(V, E)$, a sequence of $k$ weights, $w_{1} \geq$ $w_{2} \geq \ldots \geq w_{k}$, and an integer $b$.

Question: Is there a a feasible solution $\mathcal{C}=\left\{C_{1}, C_{2}, \ldots, C_{k}\right\}$ to the $\operatorname{VC}(w, b)$ problem on $G$ such that $\max _{v \in C_{i}} w(v) \leq w_{i}$ and $\left|C_{i}\right| \leq b, 1 \leq i \leq k$ ?

The Feasible- $\operatorname{VC}(w, b)$ problem is equivalent to the next well known variant of the vertex-coloring problem: 
Bounded List Vertex-Coloring Problem $\left(\mathrm{VC}\left(\phi, b_{i}\right)\right)$

Instance: A graph $G=(V, E)$, a set of colors $\mathcal{C}=\left\{C_{1}, C_{2}, \ldots, C_{k}\right\}$, a list of colors $\phi(u) \subseteq \mathcal{C}$ for each $u \in V$, and integers $b_{i}, 1 \leq i \leq k$.

QUESTION: Is there a $k$-coloring of $G$ such that each vertex $u$ is assigned a color in its list $\phi(u)$ and every color $C_{i}$ is used at most $b_{i}$ times?

Indeed, an instance of the FeAsible- $\mathrm{VC}(w, b)$ problem on a graph $G$, where we are given $k$ weights $w_{1} \geq w_{2} \geq \ldots \geq w_{k}$ and an integer $b$, can be easily transformed to the next equivalent instance of the $\mathrm{VC}\left(\phi, b_{i}\right)$ problem: is there a $k$-coloring of $G$ where each vertex $u \in V$ is assigned a color in $\phi(u)=\left\{C_{i}: w_{i} \geq w(u), 1 \leq i \leq k\right\}$ and every color $C_{i}$ is used at most $b_{i}=b$ times? A "yes" answer to this instance of the $\mathrm{VC}\left(\phi, b_{i}\right)$ problem corresponds to the existence of a feasible solution $C=$ $\left\{C_{1}, C_{2}, \ldots, C_{k}\right\}$ for the $\operatorname{VC}(w, b)$ problem of weight $W=\sum_{i=1}^{k} w_{i}$.

In an analogous way, we can use the List Vertex-Coloring $(\operatorname{VC}(\phi))$, Bounded List Edge-Coloring $\left(\mathrm{EC}\left(\phi, b_{i}\right)\right)$ and List Edge-Coloring $(\mathrm{EC}(\phi))$ problems to answer to the Feasible-VC $(w)$, Feasible-EC $(w, b)$ and Feasible-EC $(w)$ problems, respectively.

Clearly, the (bounded) list vertex and edge coloring problems generalize the (bounded) vertex and edge coloring problems. List coloring problems have been studied extensively in the literature, for many classes of underlying graphs. In the next theorem we summarize some of these results which we shall use in this thesis.

\section{Theorem 1.}

(i) Both $\mathrm{VC}\left(\phi, b_{i}\right)$ and $\mathrm{EC}\left(\phi, b_{i}\right)$ problems are polynomial on trees if the number, $k$, of colors is fixed $[21,36]$.

(ii) The $\operatorname{VC}\left(\phi, b_{i}\right)$ problem is polynomial on general graphs if $k=2[36]$.

(iii) The $\operatorname{VC}\left(\phi, b_{i}\right)$ problem is NP-complete even for chains, $|\phi(u)| \leq 2$, for all $u \in V$, and $b_{i} \leq 5,1 \leq i \leq k[23]$.

By exhaustively searching for the weights of an optimal solution of $k$ colors for a max-coloring problem, and answering to the obtained feasible coloring problem through the corresponding list coloring problem, we get the following theorem.

\section{Theorem 2.}

(i) For a fixed number of colors $k$, both $\mathrm{VC}(w, b)$ and $\mathrm{EC}(w, b)$ problems, and hence $\operatorname{VC}(w)$ and $\operatorname{EC}(w)$ problems, are polynomial on trees.

(ii) For two colors, the $\operatorname{VC}(w, b)$ problem is polynomial on general graphs.

Proof. For (i), we consider all $O\left(|V|^{k}\right)$ (resp. $O\left(|E|^{k}\right)$ ) combinations of $k$ color weights and for each combination, $w_{1}, w_{2}, \ldots, w_{k}$, we have to answer to the FEASIBLE$\operatorname{VC}(w, b)$ (resp. Feasible-EC $(w, b))$ problem. This can be done using the relation to the $\operatorname{VC}\left(\phi, b_{i}\right)$ (resp. $\left.\operatorname{EC}\left(\phi, b_{i}\right)\right)$ problem described above and the results of Theorem 1(i). An optimal solution to the $\operatorname{VC}(w, b)$ (resp. $\operatorname{EC}(w, b)$ ) problem corresponds to the combination where a feasible solution exists and the total weight $W$ is minimized. 
In a similar way, we can prove (ii), using Theorem 1(ii).

\section{Set cover and fixed cardinality bounds}

The $\operatorname{VC}(w, b)$ and $\operatorname{EC}(w, b)$ problems are also related to the well known set cover problem, where we are given a universe $U$ of elements, and a collection, $\mathcal{S}=$ $\left\{S_{1}, S_{2}, \ldots, S_{m}\right\}$, of subsets of $U$, each one of a positive cost $c_{i}, 1 \leq i \leq m$, and we ask for a minimum cost subset of $\mathcal{S}$ that covers all elements of $U$.

For an instance of the $\operatorname{VC}(w, b)$ problem on a graph $G=(V, E)$, let $U=V$ and consider $\mathcal{S}$ consisting of all the subsets of $V$, but those containing adjacent vertices, of cardinalities $j=1,2, \ldots, b$; for each such subset $S_{i} \in \mathcal{S}$ set $c_{i}=\max \left\{w(u) \mid u \in S_{i}\right\}$. Clearly, a solution to the set cover problem constructed corresponds to a solution of the $\operatorname{VC}(w, b)$ problem and a quite analogous transformation applies for the $\operatorname{EC}(w, b)$ problem. The cardinality of $\mathcal{S}$ is $O\left(b \cdot|V|^{b}\right)$, and as an $H_{b}$-approximation algorithm is known for the set cover problem [14], the next theorem follows.

Theorem 3. For a fixed bound $b$, there is an $H_{b}$-approximation algorithm for both $\mathrm{VC}(w, b)$ and $\mathrm{EC}(w, b)$ problems on general graphs.

\section{Max-coloring vs Coloring problems}

In [24], a general framework has been presented, which allows us to convert any $\rho$-approximation algorithm for the classical vertex coloring problem into an $e \cdot \rho$ approximation one for the $\mathrm{VC}(w)$ problem, on hereditary classes of graphs. The main idea of this framework is to select, in a random way, two parameters in order to round down the weight of each vertex. Hence, we can consider the input graph partitioned into subgraphs, where the vertices in each subgraph have the same weight. For each one of these subgraphs a $\rho$-approximation solution for the classical VC problem is obtained, and by concatenating the solutions found for all subgraphs we get a solution for the whole graph. Finally, this procedure is derandomized by choosing appropriate values for the two random parameters and a deterministic approximation algorithm is obtained.

This framework is used in [24] to derive an $e$-approximation algorithm for the $\mathrm{VC}(w, b)$ problem on perfect graphs. It is easy to see that it can be also applied for conversions from the $\mathrm{VC}(b), \mathrm{EC}$ and $\mathrm{EC}(b)$ problems to the $\mathrm{VC}(w, b), \mathrm{EC}(w)$ and $\mathrm{EC}(w, b)$ problems, respectively. For example, an $e$-approximation algorithm for the $\mathrm{EC}(w, b)$ problem on bipartite graphs is obtained using such a conversion, as the $\mathrm{EC}(b)$ problem is polynomial for bipartite graphs. However, this framework does not give an improvement to the approximation ratio of any other (bounded) max-coloring problem on the classes of graphs we study in this thesis, because a better ratio either is known or is presented in the next chapters.

Theorem 4. There is an e-approximation algorithm for the $\mathrm{EC}(w, b)$ problem on bipartite graphs. 


\section{Arbitrary $b$ vs $b=2$}

Another approximation result for both $\operatorname{VC}(w, b)$ and $\mathrm{EC}(w, b)$ problems on general graphs can be obtained by relating the values $O P T_{b}$ and $O P T_{2}$ of the optimal solution when the bound is $b$ and 2 , respectively. Let $W$ be the weight of solution obtained by splitting each color in $O P T_{b}$ into $\left\lceil\frac{b}{2}\right\rceil$ colors, all of cardinality at most 2. Obviously, $W \leq\left\lceil\frac{b}{2}\right\rceil O P T_{b}$. Moreover, $O P T_{2}$ is the optimal solution when all colors have cardinality at most 2 , and hence $O P T_{2} \leq W$. Thus, we have $O P T_{2} \leq\left\lceil\frac{b}{2}\right\rceil O P T_{b}$, and since both $\mathrm{VC}(w, b)$ and $\mathrm{EC}(w, b)$ problems are polynomial for general graphs and $b=2$ [10], the following theorem follows.

Theorem 5. There is a $\left\lceil\frac{b}{2}\right\rceil$-approximation algorithm for both $\mathrm{VC}(w, b)$ and $\mathrm{EC}(w, b)$ problems on general graphs.

\subsection{Notation}

In the following, we consider the coloring problems defined in Chapter 1 on a graph $G=(V, E)$, where $|V|=n$ and $|E|=m$. For the (bounded) max-vertex-coloring (resp. (bounded) max-edge-coloring) problem, a positive integer weight $w(u)$ (resp. $w(e)$ ) is associated with each vertex $u \in V$ (resp. edge $e \in E$ ). For the bounded vertex/edge-coloring problems, we are also given a cardinality bound, $b$, on the number of vertices/edges allowed to appear in each color.

We denote by $\mathcal{C}=\left\{C_{1}, C_{2}, \ldots, C_{k}\right\}$ a proper vertex- (resp. edge-) coloring of $G$ of weight $W=\sum_{i=1}^{k} w_{i}$, where $w_{i}=\max \left\{w(u) \mid u \in C_{i}\right\} \quad\left(\right.$ resp. $w_{i}=\max \{w(e) \mid e \in$ $\left.\left.C_{i}\right\}\right), 1 \leq i \leq k$. By $\mathcal{C}^{*}=\left\{C_{1}^{*}, C_{2}^{*}, \ldots, C_{k^{*}}^{*}\right\}$ we denote an optimal solution of weight $O P T=\sum_{i=1}^{k^{*}} w_{i}^{*}$, where $w_{i}^{*}, 1 \leq i \leq k^{*}$, is the weight of the $i$-th color class.

By $d_{G}(u)$ (or simply $d(u)$ ) we denote the degree of vertex $u \in V$ and by $\Delta(G)$ (or simply $\Delta$ ) the maximum degree of the graph $G$. We define the degree of each edge $e=(u, v) \in E$ as $d(u, v)=d(u)+d(v)$, while $\Delta^{\prime}(G)$ (or simply $\Delta^{\prime}$ ) denotes the maximum edge degree. For a subset of edges of $G, E^{\prime} \subseteq E$, we denote by $G\left[E^{\prime}\right]$ the subgraph of $G$ induced by the edges in $E^{\prime}$.

The following ordering and partition of the elements of a set accordingly to their weights will be used to present and analyze most of our algorithms. Given a set $S$ and a positive integer weight $w(s)$ for every element $s \in S$, we denote by $\langle S\rangle=\left\langle s_{1}, s_{2}, \ldots, s_{|S|}\right\rangle$ an ordering of $S$ such that $w\left(s_{1}\right) \geq w\left(s_{2}\right) \geq \ldots \geq w\left(s_{|S|}\right)$. For such an ordering of $S$ and a positive integer $b$, let $k_{S}=\left\lceil\frac{|S|}{b}\right\rceil$. We define the ordered b-partition of $S$, denoted by $\mathcal{P}_{S}=\left\{S_{1}, S_{2}, \ldots, S_{k_{S}}\right\}$, to be the partition of $S$ into $k_{S}$ subsets, such that $S_{i}=\left\{s_{j}, s_{j+1}, \ldots, s_{\min \{j+b-1,|S|\}}\right\}, i=1,2, \ldots, k_{S}$, $j=(i-1) b+1$. In other words, $S_{1}$ contains the $b$ heaviest elements of $S, S_{2}$ contains the next $b$ heaviest elements of $S$ and so on; clearly, $S_{k_{S}}$ contains the $|S|$ $\bmod b$ lightest elements of $S$. 


\section{Chapter 4}

\section{Max-Edge-Coloring on general and bipartite graphs}

In this chapter we present approximation results for the $\mathrm{EC}(w)$ problem on general and, mainly, on bipartite graphs. We, first, slightly improve the ratio of the known 2-approximation algorithm proposed by Kesselman and Kogan in [46] (ALGORITHM $\mathrm{KK}$ ). Our analysis of this algorithm is based on lower and upper bounds on the number of colors of any reasonable solution to the $\mathrm{EC}(w)$ problem. Next, we give a simple algorithm that returns the best among two solutions: the solution found by Algorithm KK and the one obtained by an edge-coloring of the input graph. The ratio of this simple algorithm already beats the known ratios for general and bipartite graphs.

Next, we explore an idea used in [20,25] to derive approximation ratios less than 2 for the $\mathrm{EC}(w)$ problem on bipartite graphs of $\Delta \leq 7$. The same ideas has been also used in $[20,51]$ to derive an 8/7-approximation algorithm for the $\mathrm{VC}(w)$ problem for bipartite graphs. In general, we find a number of solutions for a bipartite graph $G$ by concatenating partial solutions for disjoint edge induced subgraphs of $G$ and we select the best among them. Using this idea we present a series of four algorithms of different approximation ratios for the $\mathrm{EC}(w)$ problem on bipartite graphs. The approximation ratios of our three first algorithms depend on the maximum degree of the input graph. The last of our algorithms achieves an 1.74 approximation ratio for this problem and it is the first one that improves the known ratio of 2 .

Finally, in Section 4.4 we prove that the $\mathrm{EC}(w)$ problem is NP-complete even on bi-valued complete graphs. Moreover, we present an asymptotic 4/3-approximation algorithm for general bi-valued graphs.

\subsection{Preliminaries}

The $\operatorname{EC}(w)$ problem is polynomial for graphs of maximum degree $\Delta=2$. This result follows from the same variant of the $\operatorname{VC}(w)$ problem. In fact, an $O\left(|V|^{2}\right)$ algorithm for the $\mathrm{VC}(w)$ problem on chains has been presented in [25], which can be easily adapted to graphs of maximum degree $\Delta=2$ (that are collections of chains 
and cycles). If $G$ is a graph of maximum degree $\Delta(G)=2$, then its line graph $L(G)$ is also a graph with $\Delta(L(G))=2$ and the following theorem holds.

Theorem 6. An optimal solution to the $\mathrm{EC}(w)$ problem for graphs of maximum degree $\Delta=2$ can be found in $O\left(|E|^{2}\right)$ time.

To bound the number of colors in any solution to the $\mathrm{EC}(w)$ problem we can restrict only on a specific subset of them. We shall call a solution $\mathcal{C}=\left\{C_{1}, C_{2}, \ldots, C_{k}\right\}$ to the $\mathrm{EC}(w)$ problem nice if: (i) $w_{1} \geq w_{2} \geq \ldots \geq w_{k}$, and (ii) each color $C_{i}$ is maximal in the subgraph $G\left[\bigcup_{j=i}^{k} C_{j}\right]$. Due to the next proposition we consider, w.l.o.g., any, suboptimal or optimal, solution to the $\mathrm{EC}(w)$ problem to be a nice one.

Proposition 2. Any solution to the $\mathrm{EC}(w)$ problem can be transformed into a nice one, without increasing its total weight. For the number of colors, $k$, in such a solution it holds that $\Delta \leq k \leq \Delta^{\prime}-1 \leq 2 \Delta-1$.

Proof. Obviously, any solution to the $\mathrm{EC}(w)$ problem consists of at least $\Delta$ colors, since there is at least one vertex with exactly $\Delta$ adjacent edges, and these $\Delta$ edges belong to different colors.

Assume that an optimal solution consists of $\Delta^{\prime}$ or more colors. Consider those colors sorted in non-increasing order of their weights. Each edge of $G$ has at most $\Delta^{\prime}-2$ neighbor edges. So, for each edge $e$ in any color $C_{i}, i \geq \Delta^{\prime}$, there is at least one color, $C_{j}, j<\Delta^{\prime}$, such that edge $e$ can be moved to $C_{j}$ without increasing $C_{j}$ 's weight.

The last part of the inequality follows directly by the definition of $\Delta^{\prime}$.

The most interesting and general result for the $\mathrm{EC}(w)$ problem is due to Kesselman and Kogan [46] who proposed the following greedy algorithm:

\section{Algorithm KK}

1: Let $\langle E\rangle=\left\langle e_{1}, e_{2}, \ldots, e_{m}\right\rangle$;

2: for $i=1$ to $m$ do

3: $\quad$ Insert $e_{i}$ into the first color not containing other edges adjacent to $e_{i}$;

4: end for

In [46], it has been shown that Algorithm KK is a 2-approximation one and an example has been presented yielding an approximation ratio of $2-\frac{1}{\Delta}$. By a slightly tighter analysis using Proposition 2 we prove here the next lemma.

Lemma 1. Algorithm KK achieves an approximation ratio of $\min \left\{2-\frac{w_{1}^{*}}{O P T}\right.$, $\left.2-\frac{1}{\Delta}\right\}$ for the $\mathrm{EC}(w)$ problem.

Proof. The solution, $\mathcal{C}=\left\{C_{1}, C_{2}, \ldots, C_{k}\right\}$, that Algorithm KK returns is, by its construction, a nice one. Let $e$ be the first edge that the algorithm inserts into color $C_{i}$; then it holds that $w_{i}=w(e)$. Let $E_{i}$ be the set of edges preceding $e$ in $\langle E\rangle$ and edge $e$ itself, i.e., $E_{i}=\left\{e_{1}, e_{2}, \ldots, e_{i-1}\right\}$, and $\Delta_{i}$ be the maximum degree 
of the subgraph $G\left[E_{i}\right]$. The optimal solution for the $\operatorname{EC}(w)$ problem on the graph $G\left[E_{i}\right]$ contains $i^{*} \geq \Delta_{i}$ colors each one of weight at least $w_{i}$, that is $w_{i} \leq w_{i^{*}}^{*}$. By Proposition 2, the colors constructed by Algorithm KK for the graph $G\left[E_{i}\right]$ are $i \leq 2 \Delta_{i}-1 \leq 2 i^{*}-1$, that is $i^{*} \geq\left\lceil\frac{i+1}{2}\right\rceil$. Hence, $w_{i} \leq w_{i^{*}}^{*} \leq w_{\left\lceil\frac{i+1}{2}\right\rceil}^{*}$.

Summing up the above bounds for all $w_{i}$ 's, $1 \leq i \leq k \leq 22 \Delta-1$, we obtain $W \leq \sum_{i=1}^{2 \Delta-1} w_{i} \leq w_{1}^{*}+2\left(\sum_{i=2}^{\Delta} w_{i}^{*}\right)=2\left(\sum_{i=1}^{\Delta} w_{i}^{*}\right)-w_{1}^{*}$. As $k^{*} \geq \Delta$, it follows that $\sum_{i=1}^{\Delta} w_{i}^{*} \leq O P T$. Therefore, $\frac{W}{O P T} \leq 2-\frac{w_{1}^{*}}{O P T}$ and also $\frac{W}{O P T} \leq \frac{2 \sum_{i=1}^{\Delta} w_{i}^{*}-w_{1}^{*}}{\sum_{i=1}^{\Delta} w_{i}^{*}} \leq$ $2-\frac{w_{1}^{*}}{\sum_{i=1}^{\Delta} w_{i}^{*}} \leq 2-\frac{w_{1}^{*}}{\Delta \cdot w_{1}^{*}}=2-\frac{1}{\Delta}$.

It is well known that a $(\Delta+1)$-coloring of a general graph and a $\Delta$-coloring of a bipartite graph can be found in polynomial time. In fact, a $(\Delta+1)$-coloring of a general graph can be found in $O(\min \{|V| \Delta \log |V|,|E| \sqrt{|V| \log |V|}\})$ time [29], while a $\Delta$-coloring of a bipartite graph in $O(|E| \log \Delta)$ time [16]. Such a coloring yields a feasible, but in general not optimal, solution for the $\mathrm{EC}(w)$ problem.

Intuitively, a solution obtained this way will be close to an optimal one when the edge weights are close to each other, while AlgORITHM KK performs better in the opposite case. Next theorem follows by selecting the best among the two solutions found by ALGORIThm KK and a $(\Delta+1)-$ or $\Delta$-coloring of the input graph.

Theorem 7. There is an approximation algorithm for the $\mathrm{EC}(w)$ problem of ratio $2-\frac{2}{\Delta+2}$ for general graphs and $2-\frac{2}{\Delta+1}$ for bipartite graphs.

Proof. By Lemma 1, a solution found by Algorithm KK is of weight $W \leq 2 O P T-$ $w_{1}^{*}$. Any $\Delta$-coloring of a bipartite graph yields a solution for the $\mathrm{EC}(w)$ problem of weight $W \leq \Delta \cdot w_{1}^{*}$, since $w_{1}^{*}$ equals to the weight of the heaviest edge of the graph, and hence to the weight of the heaviest color of any solution created for this graph. Multiplying both sides of the second inequality with $1 / \Delta$ and adding this to the first one we obtain: $\left(1+\frac{1}{\Delta}\right) W \leq 2 O P T$, that is $W \leq\left(2-\frac{2}{\Delta+1}\right) O P T$.

For general graphs we simply consider a $(\Delta+1)$-coloring, instead of a $\Delta$-coloring, of the input graph.

For the tightness of our analysis for bipartite graphs, consider the instance of the $\mathrm{EC}(w)$ problem shown in Figure 4.1(a). The weight of an optimal solution to this instance is $2+\epsilon$ (Figure 4.1(b)), the weight of the solution of AlGorithm $\mathrm{KK}$ is 3 (Figure 4.1(c)), and the weight of a solution found by a $\Delta$-coloring of the input graph (Figure 4.1(d)) is also 3. By selecting either solution a ratio of $\frac{3}{2+\epsilon} \simeq \frac{3}{2}=2-\frac{2}{\Delta+1}$ is attained. 


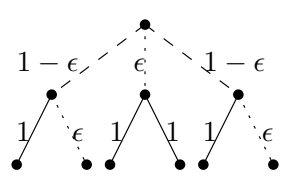

$(a)$

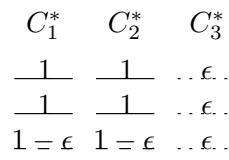

(b)

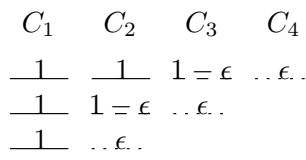

(c)

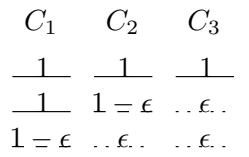

$(d)$

Figure 4.1: A tight example for the $\left(2-\frac{2}{\Delta+1}\right)$-approximation ratio of Theorem 7 for bipartite graphs $(\Delta=3, \epsilon<<1)$.

Figure 4.2(a) shows an analogous example for the approximation ratio of Theorem 7 for general graphs. The solution created by ALGORITHM KK (Figure 4.2(c)) has weight $4-3 \epsilon$, while the solution obtained by a $(\Delta+1)$-coloring (Figure $4.2(\mathrm{~d})$ ) has weight 4 . By selecting the first solution a ratio of $\frac{4-3 \epsilon}{\frac{5}{2}} \simeq \frac{8}{5}=2-\frac{2}{\Delta+2}$ is attained, since the optimal solution (Figure 4.2(b)) has weight 5/2.

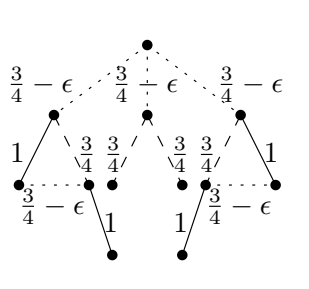

(a)

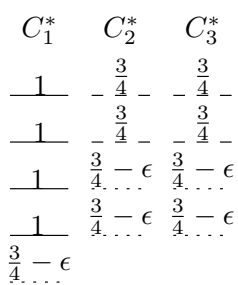

(b)

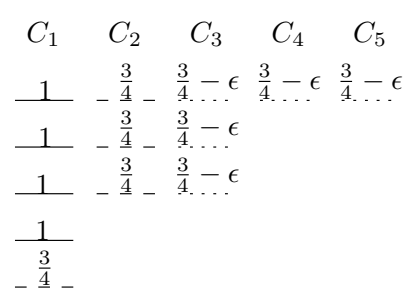

(c)

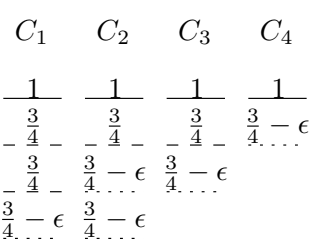

$(d)$

Figure 4.2: A tight example for the $\left(2-\frac{2}{\Delta+2}\right)$-approximation ratio of Theorem 7 for general graphs $(\Delta=3, \epsilon<<1)$.

The ratios of Theorem 7 are better than $2-\frac{1}{\Delta}$ for any $\Delta \geq 3$. More interestingly, the ratios for bipartite graphs are better than the $(2 \Delta-1) / 3$ approximation ratio proposed in [22], for any $\Delta$, as well as than the ratios of the algorithm presented in [25], for $\Delta \geq 4$.

\section{2 $f(\Delta)$-approximation algorithms for bipartite graphs}

The known approximation algorithms $[20,25]$ of ratios less than 2 for the $\operatorname{EC}(w)$ problem on a bipartite graph $G=(V, E)$ are based on the following general idea: Consider an ordering $\langle E\rangle=\left\langle e_{1}, e_{2}, \ldots, e_{m}\right\rangle$ of the edges of $G$, and let $E_{p, q}=$ $\left\{e_{p}, e_{p+1}, \ldots, e_{q}\right\}$. Repeatedly, partition the graph $G$ into three edge induced subgraphs: the graph $G\left[E_{1, p}\right]$ induced by the $p$ heaviest edges of $G$, the graph $G\left[E_{p+1, q}\right]$, induced by the next $q-p$ edges of $G$, and the graph $G\left[E_{q+1, m}\right]$, induced by the $m-q$ lightest edges of $G$. Find a solution for the whole graph $G$ by considering the $\mathrm{EC}(w)$ problem on these three subgraphs and return the best among the solutions 
found. Depending on how the problem is handled for each subgraph and the analysis followed, this general idea leads to different algorithms and approximation ratios. Notice that the same approach is employed by the $8 / 7$-approximation algorithm for the $\operatorname{VC}(w)$ problem on bipartite graphs $[20,51]$.

In this section we further explore this idea, and we present a series of three $f(\Delta)$-approximation algorithms, each one improving the ratios of the previous ones. Our first algorithm gives approximation ratios better than the previous known for bipartite graphs of maximum degree $4 \leq \Delta \leq 12$, but its ratio becomes greater than 2 for $\Delta \geq 13$. The approximation ratios of our second and third algorithms are smaller than 2 for any $\Delta$. Our third algorithm achieves the best ratios for bipartite graphs of maximum degree $\Delta \geq 7$. However, both these algorithms give ratios that tend asymptotically to 2 as $\Delta$ increases.

To describe our algorithms, let us introduce some additional notation. We denote by $(p, q), 0 \leq p<q \leq m$, a partition of $G$ into subgraphs $G\left[E_{1, p}\right], G\left[E_{p+1, q}\right]$ and $G\left[E_{q+1, m}\right]$; by convention, we define $E_{1,0}=\emptyset, E_{m+1, m}=\emptyset$ and $E_{0, q}=E_{1, q}$. By $\Delta_{p, q}$ we denote the maximum degree of the subgraph $G\left[E_{p, q}\right]$, and by $d_{p, q}(u)$ the degree of $u \in V$ in $G\left[E_{p, q}\right]$. We denote by $p_{\delta}$ the maximum index such that $\Delta_{1, p_{\delta}}=\delta$. It is clear that $p_{1}<p_{2}<\ldots<p_{\Delta}=m$.

Finally, henceforth in this section, the following proposition will be useful.

Proposition 3. Given a graph $G=(V, E)$ and a subset $A \subseteq V$, we can determine if there is a matching $M$ in $G$ saturating all vertices in $A$ in $O\left(|V|^{2.5}\right)$ time.

Proof. Consider the graph $G^{\prime}=(X, Y)$ constructed by adding to $G$ an additional vertex, if $|V|$ is odd, and all the missing edges between the vertices $X-A$ (i.e., the vertices $X-A$ induce a clique in $G^{\prime}$ ). If there exists a perfect matching in $G^{\prime}$, then there exists a matching in $G$ saturating all vertices in $A$, since no edges adjacent to $A$ have been added in $G^{\prime}$.

Conversely, if there exists a matching $M$ in $G$ saturating all vertices in $A$, then there exists a perfect matching in $G^{\prime}$, consisting of the edges of $M$ plus the edges of a perfect matching in the complete subgraph of $G^{\prime}$ induced by its vertices that are not saturated by $M$.

Therefore, in order to determine if there exists a matching $M$ in $G$ it is enough to check if there exists a perfect matching in $G^{\prime}$. It is well known that this last check can be done in $O\left(|V|^{2.5}\right)$ time [50].

\section{Bipartite graphs of small maximum degree}

In this section, we present a first approximation algorithm for the $\mathrm{EC}(w)$ problem on bipartite graphs, exploiting the idea of splitting the input bipartite graph into edge induced subgraphs. In fact, our algorithm generalizes the $7 / 6$-approximation algorithm for bipartite graphs of $\Delta=3$, proposed in [20], such that: (a) it remains polynomial for general bipartite graphs, and (b) it achieves a substantial improvement of the best known approximation ratios for the $\mathrm{EC}(w)$ problem on bipartite graphs of maximum degree $4 \leq \Delta \leq 12$. 
In general, for each partition $(p, q), p=1,2, \ldots, p_{\Delta-1}, q=p+1, \ldots, m$, our algorithm checks the existence of two different specific sets of edges in graph $G\left[E_{p+1, q}\right]$ and for each one of them, if there exist, it computes a solution to the $\mathrm{EC}(w)$ problem on graph $G$. The algorithm returns the best among all the solutions found.

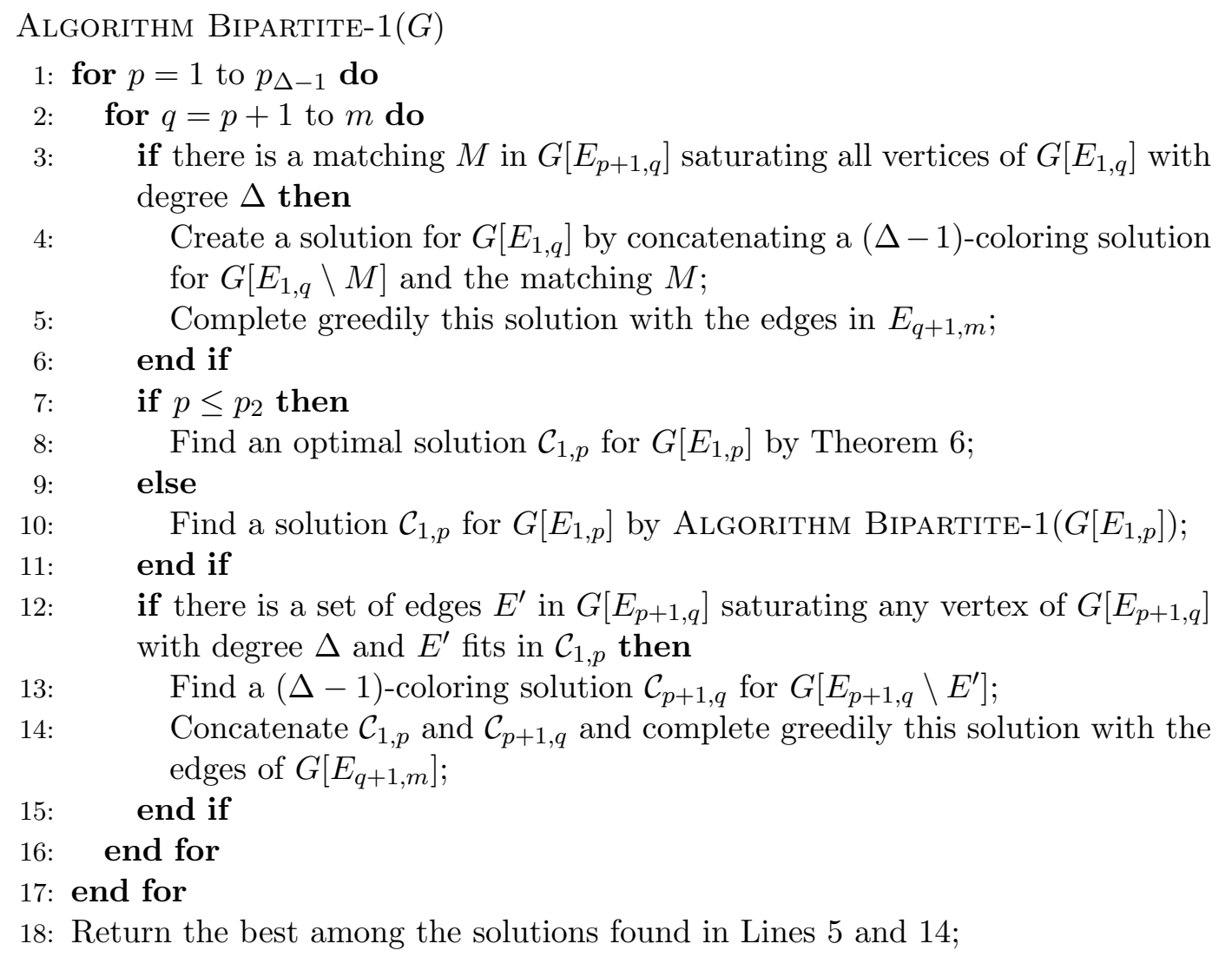

In Line 3 the algorithm checks the existence of a matching $M$ in $G\left[E_{p+1, q}\right]$ saturating all vertices of degree $\Delta$ in $G\left[E_{1, q}\right]$. It holds that $\Delta_{1, p} \leq \Delta-1$, since $1 \leq p \leq p_{\Delta-1}$, and therefore the vertices of degree $\Delta$ in subgraphs $G\left[E_{1, q}\right]$ and $G\left[E_{p+1, q}\right]$ are the same. Hence, the existence of $M$ can be checked by applying Proposition 3 on the graph $G\left[E_{p+1, q}\right]$ with $A$ being the set of vertices of degree $\Delta$ in $G\left[E_{1, q}\right]$.

In Line 12 the algorithm checks the existence of a set of edges $E^{\prime}$ in $G\left[E_{p+1, q}\right]$ saturating all vertices of degree $\Delta$ in $G\left[E_{p+1, q}\right]$ and, moreover, fitting the solution $\mathcal{C}_{1, p}$. Consider the subgraph $H$ of $G\left[E_{p+1, q}\right]$ induced by its vertices of degree $d_{1, p}(u) \leq$ $\Delta_{1, p}-1$. Note that, by construction, each edge in $H$ fits in a color of the solution $\mathcal{C}_{1, p}$. Let $A$ be the subset of vertices of $H$ of degree $d_{p+1, q}(u)=\Delta$, i.e. the set of vertices which we want to saturate, and $B$ the subset of vertices in $A$ of degree $d_{H}(u)=1$. For each vertex $u \in B$ we can clearly insert the single edge $(u, v)$ in $E^{\prime}$. Let $H^{\prime}$ be the subgraph of $H$ induced by its vertices but those in $B$ and $A^{\prime} \subseteq A$ be the subset of vertices of $A$ that are not saturated by the edges already in $E^{\prime}$. It is now enough to find a matching on $H^{\prime}$ that saturates each vertex in $A^{\prime}$, using 
Proposition 3. Adding the edges of this matching in $E^{\prime}$ we get a set that saturates each vertex of degree $\Delta$ in graph $G\left[E_{p+1, q}\right]$.

In Lines 5 and 14 the algorithm completes a partial solution by examining the remaining lightest edges one by one and assigning them to the first color they fit in. If such a color does not exist, then a new one is created. As both partial solutions consist of at most $2 \Delta-1$ colors, the complete solutions obtained will consist also of at most $2 \Delta-1$ colors, according to the arguments in the proof of Proposition 2.

The following lemma provides bounds to the weight $W$ of the solution obtained by Algorithm Bipartite- 1 . We denote by $\varrho_{\Delta}$ the approximation ratio of our algorithm for a graph of maximum degree $\Delta$. By definition, $\varrho_{1}=\varrho_{2}=1$, since, by Theorem 6 , the $\mathrm{EC}(w)$ problem for graphs of maximum degree 1 or 2 can be solved in polynomial time. Recall also that we consider the colors of an optimal solution in non-increasing order with respect to their weights, i.e., $w_{1}^{*} \geq w_{2}^{*} \geq \cdots \geq w_{k^{*}}^{*}$.

Lemma 2. Algorithm Bipartite-1 returns a solution of weight:

$$
\begin{aligned}
W \leq & \min \left\{(\Delta-1) \cdot w_{1}^{*}+w_{\Delta}^{*}+(\Delta-1) \cdot w_{\Delta+1}^{*},\right. \\
& \left.\min _{1 \leq \delta \leq \Delta-1}\left\{\varrho_{\delta} \cdot \sum_{i=1}^{\delta} w_{i}^{*}+(\Delta-1) \cdot w_{\delta+1}^{*}+(\Delta-\delta) \cdot w_{\Delta+\delta}^{*}\right\}\right\}
\end{aligned}
$$

Proof. For the first term in the righthand side of the lemma's inequality, we consider the solution obtained in Lines 3 to 6 of Algorithm Bipartite- $1(G)$ in the iteration $(p, q)$ where $w\left(e_{p+1}\right)=w_{\Delta}^{*}$ and $w\left(e_{q+1}\right)=w_{\Delta+1}^{*}$ (note that if $q=m$, then $k^{*}=\Delta$ and $w_{i}^{*}=0$, for each $\left.i \geq \Delta+1\right)$. In this iteration the matching $M$ exists, since in the optimal solution the edges in $E_{1, q}$ belong to at most $\Delta$ colors and the $\Delta$-th color contains edges of weight at most $w\left(e_{p+1}\right)$. The weight of $M$ is at most $w_{\Delta}^{*}$, while the weight of the solution found for $G\left[E_{1, q} \backslash M\right]$ is bounded by $(\Delta-1) \cdot w_{1}^{*}$. The greedy step in Line 5 creates at most $\Delta-1$ colors, each one of weight at most $w_{\Delta+1}^{*}$. Therefore, $W \leq(\Delta-1) \cdot w_{1}^{*}+w_{\Delta}^{*}+(\Delta-1) \cdot w_{\Delta+1}^{*}$.

For the second term in the righthand side of the lemma's inequality, consider the solutions obtained in Lines 7 to 15 of Algorithm Bipartite- $1(G)$ for $\Delta-1$ different iterations $(p, q)$. For $p \leq p_{\delta}$, consider the iteration $q$ where $w\left(e_{p+1}\right)=w_{\delta+1}^{*}$ and $w\left(e_{q+1}\right)=w_{\Delta+\delta}^{*}$. In this iteration, the set of edges $E^{\prime}$ exists, since in the optimal solution the edges in $E_{p+1, q}$ belong to at most $\Delta-1$ colors.

If $\delta=1$ or 2 , the algorithm creates an optimal solution for $G\left[E_{1, p}\right]$ using Theorem 6 , since $\Delta_{1, p} \leq 2$.

If $q \geq 3$, the algorithm creates an approximation solution for $G\left[E_{1, p}\right]$.

In both cases, the edges in $E_{1, p}$ are a subset of the edges of the $\delta$ heaviest colors in the optimal solution. Thus, it holds that $\mathcal{C}_{1, p} \leq \varrho_{\delta} \cdot \sum_{i=1}^{\delta} w_{i}^{*}$, where $\varrho_{1}=\varrho_{2}=1$.

The weight of the solution found for $G\left[E_{p+1, q} \backslash E^{\prime}\right]$ is bounded by $(\Delta-1) \cdot w_{\delta+1}^{*}$, since $\Delta\left(G\left[E_{p+1, q} \backslash E^{\prime}\right]\right) \leq \Delta-1$ and for each edge $e \in E_{p+1, q} \backslash E^{\prime}$ it holds that $w(e) \leq w_{\delta+1}^{*}$.

The greedy step in Line 14 creates at most $\Delta-\delta$ colors, each one of weight at most $w_{\Delta+\delta}^{*}$.

Summing up the weights of these three partial solutions the lemma follows. 
Algorithm Bipartite- $1(G)$ performs $O\left(|E|^{2}\right)$ iterations. In Line 10 of (some of) those iterations the algorithm is called recursively for the graph $G\left[E_{1, p}\right]$. In this call, for each combination of $p^{\prime}, q^{\prime}, 1 \leq p^{\prime}<q^{\prime} \leq p$, the graph $G_{1, p}$ is partitioned into the three edge induced subgraphs $G_{1, p^{\prime}}, G_{p^{\prime}+1, q^{\prime}}$ and $G_{q^{\prime}+1, p}$.

The solution for $G\left[E_{1, p^{\prime}}\right]$ has been already computed in a previous iteration of Algorithm Bipartite- $1(G)$ in which $p=p^{\prime}$. By storing this solution we avoid its recursive recomputation. Thus, Algorithm Bipartite- $1\left(G\left[E_{1, p}\right]\right)$ performs in total also $O\left(|E|^{2}\right)$ iterations. By Proposition 3, the matching $M$ and the set $E^{\prime}$ can be computed in polynomial time, and this derives a polynomial overall complexity.

To obtain the ratio of Algorithm Bipartite- 1 we consider the $\Delta$ bounds on $W$ and we combine them in an optimal way. For instance, for $\Delta=4$, Algorithm BIPARTITE-1 returns a solution of weight $W$, for which holds that:

$$
\begin{aligned}
& W \leq 3 \cdot w_{1}^{*}+w_{4}^{*}+3 \cdot w_{5}^{*} \\
& W \leq w_{1}^{*}+3 \cdot w_{2}^{*}+3 \cdot w_{5}^{*} \\
& W \leq w_{1}^{*}+w_{2}^{*}+3 \cdot w_{3}^{*}+2 \cdot w_{6}^{*} \\
& W \leq 7 / 6 \cdot\left(w_{1}^{*}+w_{2}^{*}+w_{3}^{*}\right)+3 \cdot w_{4}^{*}+w_{7}^{*}
\end{aligned}
$$

Multiplying these inequalities by 44/458,66/458, 99/458 and 138/458, respectively, and adding them we obtain a ratio $\varrho_{4}=458 / 347 \simeq 1.32$ for the $\mathrm{EC}(w)$ problem on bipartite graphs with maximum degree $\Delta=4$, that dominates the 1.61 ratio by [25]. In the same way, we can compute the ratio $\varrho_{\Delta}$ for different values of $\Delta$.

Table 4.1 summarizes the approximation ratios achieved by our algorithm, as well as the previous best known ratios for $\Delta=3,4, \ldots, 12$.

\begin{tabular}{c||cc}
\hline \hline$\Delta$ & Best known ratio & Our ratio \\
\hline \hline 3 & $1.17[20]$ & 1.17 \\
4 & $1.61[25]$ & 1.32 \\
5 & $1.75[25]$ & 1.45 \\
6 & $1.86[25]$ & 1.56 \\
7 & $1.95[25]$ & 1.65 \\
8 & $2[46]$ & 1.74 \\
9 & $2[46]$ & 1.81 \\
10 & $2[46]$ & 1.87 \\
11 & $2[46]$ & 1.93 \\
12 & $2[46]$ & 1.98 \\
\hline \hline
\end{tabular}

Table 4.1: Summary of the approximation ratios achieved by Algorithm BIPARTITE- 1 vs. previous best known ratios for $\Delta=3, \ldots, 12$.

Algorithm Bipartite- 1 achieves better results than the approximation ratio $(2 \Delta-1) / 3$ proposed in [22], for any $\Delta$, as well as than the 2-approximation algorithm given in [46], for bipartite graphs with $\Delta \leq 12$. Furthermore, for bipartite graphs of $4 \leq \Delta \leq 7$ our algorithm improves the best known results given in [25]. For 
bipartite graphs of maximum degree $\Delta=3$, Algorithm Bipartite- 1 reduces to the $7 / 6$-approximation algorithm proposed in [20].

\section{Bipartite graphs of arbitrary maximum degree}

In this section we present a new algorithm for the $\mathrm{EC}(w)$ problem on bipartite graphs. It also produces $O\left(|E|^{2}\right)$ different solutions by splitting the input graph into edge induced subgraphs and chooses the best of them. The new algorithm beats the previous ratios for bipartite graphs for any $\Delta \geq 9$ and it is the first one of this kind yielding approximation ratios that tends asymptotically to 2 as $\Delta$ increases.

In general, for each $p=1,2, \ldots, p_{2}$, our algorithm examines a partition of the graph $G$ into two edge induced subgraphs: $G\left[E_{1, p}\right]$ of $\Delta_{1, p} \leq 2$ and $G\left[E_{p+1, m}\right]$. For each one of these partitions, the algorithm computes a solution to the $\operatorname{EC}(w)$ problem on graph $G$.

Moreover, for each partition $(p, q), p=1,2, \ldots, p_{2}, q=p+1, \ldots, m$, our algorithm checks the existence of a set of edges in graph $G\left[E_{p+1, q}\right]$, just the same to the set required to ALGORITHM BIPARTITE-1. If such a set of edges exists the algorithm computes a solution to the $\operatorname{EC}(w)$ problem on graph $G$.

The algorithm computes one more solution by finding a $\Delta$-coloring of the original graph $G$ and returns the best among all the solutions found.

\section{Algorithm Bipartite-2}

1: Find a $\Delta$-coloring solution $\mathcal{C}_{1, m}^{0}$ for $G$;

2: for $p=1$ to $p_{2}$ do

3: $\quad$ Find an optimal solution $\mathcal{C}_{1, p}^{1}$ for $G\left[E_{1, p}\right]$;

4: $\quad$ Find a $\Delta$-coloring solution $\mathcal{C}_{p+1, m}^{1}$ for $G\left[E_{p+1, m}\right]$;

5: $\quad$ Concatenate $\mathcal{C}_{1, p}^{1}$ and $\mathcal{C}_{p+1, m}^{1}$;

6: $\quad$ for $q=p+1$ to $m$ do

7: $\quad$ Find an optimal solution $\mathcal{C}_{1, p}^{2}$ for $G\left[E_{1, p}\right]$;

8: $\quad$ if there is a set of edges $E^{\prime}$ in $G\left[E_{p+1, q}\right]$ saturating any vertex of $G\left[E_{p+1, q}\right]$ with degree $\Delta_{1, q}$ and $E^{\prime}$ fits in $\mathcal{C}_{1, p}^{2}$ then

9: $\quad$ Find a solution $\mathcal{C}_{p+1, q}^{2}$ by a $\left(\Delta_{1, q}-1\right)$-coloring of $G\left[E_{p+1, q} \backslash E^{\prime}\right]$;

10: $\quad$ Find a solution $\mathcal{C}_{q+1, m}^{2}$ by a $\Delta$-coloring of $G\left[E_{q+1, m}\right]$;

11: $\quad$ Concatenate $\mathcal{C}_{1, p}^{2}, \mathcal{C}_{p+1, q}^{2}$ and $\mathcal{C}_{q+1, m}^{2}$;

12: $\quad$ end if

13: $\quad$ end for

14: end for

15: Return the best among the solutions found in Lines 1, 5 and 11;

Note that the main difference between the new algorithm and earlier ones, like the algorithm proposed in [25] as well as Algorithm Bipartite-1, is that it keeps always the maximum degree $\Delta_{1, p}$ of the subgraph $G\left[E_{1, p}\right]$ at most two and thus it always computes an optimal solution for $G\left[E_{1, p}\right]$. Recall that in the earlier algorithm, $\Delta_{1, p}$ is allowed to take values form 1 to $\Delta$, and thus there are cases where only an 
approximate solution can be found for $G\left[E_{1, p}\right]$. Due to this fact, the ratio of those algorithms can increase to $O(\Delta)$.

The existence of the set of edges $E^{\prime}$ in Line 8 of the algorithm can be determined in polynomial time in the same way as the same set in Algorithm Bipartite-1.

Theorem 8. Algorithm BIPARTITE-2 is a $\left(\frac{2 \Delta^{3}}{\Delta^{3}+\Delta^{2}+\Delta-1}\right)$-approximation one for the $\mathrm{EC}(w)$ problem on bipartite graphs.

Proof. The solution obtained by a $\Delta$-coloring of the input graph computed in Line 1 of the algorithm is of weight $W \leq \mathcal{C}_{1, m}^{0} \leq \Delta \cdot w_{1}^{*}$, since $w_{1}^{*}$ equals to the heaviest edge of the graph.

In Lines 3-5, consider the solutions obtained in the iterations where $w\left(e_{p+1}\right)=$ $w_{z}^{*}$, for $z=2,3$. In both cases it holds that $\Delta_{1, p} \leq 2$. An optimal solution is computed for $G_{1, p}$ of weight $\mathcal{C}_{1, p}^{1} \leq \sum_{i=1}^{z-1} w_{i}^{*}$, since the edges in $E_{1, p}$ are a subset of the edges that appear in the $z-1$ heaviest colors of the optimal solution. Moreover, a $\Delta$-coloring is built for $G\left[E_{p+1, m}\right]$ of weight $\mathcal{C}_{p+1, m}^{1} \leq \Delta \cdot w_{z}^{*}$, since $e_{p+1}$ is the heaviest edge of this subgraph. Therefore,

$$
W \leq \sum_{i=1}^{z-1} w_{i}^{*}+\Delta \cdot w_{z}^{*}, \text { for } z=2,3 .
$$

In Lines $7-12$, consider the solutions obtained in the iterations $(p, q)$ where $w\left(e_{p+1}\right)=w_{3}^{*}$ and $w\left(e_{q+1}\right)=w_{z}^{*}$, for $4 \leq z \leq \Delta$. In these iterations the set of edges $E^{\prime}$ exists, since in the optimal solution the edges in $E_{1, q}$ belong in at most $\Delta_{1, q} \leq z-1$ colors. The edges of $E^{\prime}$ are lighter than the edges in $E_{1, p}$, and thus it is possible to add them in $\mathcal{C}_{1, p}^{2}$ without increasing its weight. Thus, using the same arguments as for the weight of $\mathcal{C}_{1, p}^{1}$, it holds that $\mathcal{C}_{1, p}^{2} \leq w_{1}^{*}+w_{2}^{*}$. The heaviest edges in $G\left[E_{p+1, q} \backslash E^{\prime}\right]$ and $G\left[E_{q+1, m}\right]$ are equal to $w_{3}^{*}$ and $w_{z}^{*}$, respectively. Hence, we have that $\mathcal{C}_{p+1, q}^{2} \leq\left(\Delta_{1, q}-1\right) \cdot w_{3}^{*} \leq(z-2) \cdot w_{3}^{*}$ and $\mathcal{C}_{q+1, m}^{2} \leq \Delta \cdot w_{z}^{*}$. Therefore,

$$
W \leq w_{1}^{*}+w_{2}^{*}+(z-2) \cdot w_{3}^{*}+\Delta \cdot w_{z}^{*}, \text { for } 4 \leq z \leq \Delta .
$$

As the algorithm returns the best among the solutions found, we have $\Delta$ bounds on the weight $W$ of this best solution, i.e.,

$$
\begin{aligned}
& W \leq \Delta \cdot w_{1}^{*} \\
& W \leq \sum_{i=1}^{z-1} w_{i}^{*}+\Delta \cdot w_{z}^{*}, \text { if } z=2 \text { or } 3, \text { and } \\
& W \leq w_{1}^{*}+w_{2}^{*}+(z-2) \cdot w_{3}^{*}+\Delta \cdot w_{z}^{*}, \text { if } 4 \leq z \leq \Delta .
\end{aligned}
$$

To derive our ratio we denote by $c_{j z}, 1 \leq z, j \leq \Delta$, the coefficient of the weight $w_{j}^{*}$ in the $z$-th bound on $W$ and we find the solution of the system of linear equations $\mathbf{C} \cdot \mathbf{x}^{T}=\mathbf{1}^{T}$, that is

$$
x_{z}=\left\{\begin{array}{lll}
\frac{\Delta^{2}-1}{2^{2}}, & \text { if } & z=1 \\
\frac{\Delta^{2}+1}{2 \Delta^{2}}, & \text { if } & z=2 \\
\frac{5-\Delta}{2 \Delta}, & \text { if } & z=3 \\
\frac{1}{\Delta}, & \text { if } & 4 \leq z \leq \Delta .
\end{array}\right.
$$


By multiplying both sides of the $i$-th bound on $W$ by $x_{i}$ and adding all of them we have $\sum_{z=1}^{\Delta} x_{z} \cdot W \leq w_{1}^{*}+w_{2}^{*}+\ldots+w_{\Delta}^{*} \leq O P T$. Hence,

$$
\begin{aligned}
\frac{W}{O P T} & \leq \frac{1}{\sum_{z=1}^{\Delta} x_{z}} \leq \frac{2 \Delta^{3}}{\left(\Delta^{2}-1\right)+\Delta(\Delta+1)+\Delta^{2}(5-\Delta)+2 \Delta^{2}(\Delta-3)} \\
& =\frac{2 \Delta^{3}}{\Delta^{3}+\Delta^{2}+\Delta-1} .
\end{aligned}
$$

The complexity of Algorithm Bipartite-2 is dominated by the check in Line 8 , which by Proposition 3 can be done in polynomial time. This check runs for $O\left(|E|^{2}\right)$ different combinations of weights.

\section{Improvement for bipartite graphs of arbitrary maximum degree}

In this section we further explore the limitations of the same idea of repeatedly partitioning the graph into three edge induced subgraphs of heaviest, medium and lightest edges. We present a new algorithm for the $\mathrm{EC}(w)$ problem on bipartite graphs, which improves all the previous ratios for $\Delta \geq 7$.

For a partition $(p, q)$ of $G$, we call critical matching a matching $M \subseteq E_{p+1, q}$ which saturates all the vertices of $G\left[E_{1, q}\right]$ of degree $\Delta_{1, q}$. The proposed algorithm relies on the existence of such a critical matching $M$ : a solution for the subgraph $G\left[E_{1, q}\right]$ is found by concatenating a $\left(\Delta_{1, q}-1\right)$-coloring solution for the subgraph $G\left[E_{1, q} \backslash M\right]$ and the matching $M$, if exists, and by a $\Delta_{1, q}$-coloring of the subgraph $G\left[E_{1, q}\right]$, otherwise. For each partition $(p, q)$, the algorithm computes a solution for the input graph $G$ by concatenating a solution for $G\left[E_{1, q}\right]$ and a $\Delta$-coloring solution for $G\left[E_{q+1, m}\right]$. The algorithm computes also a $\Delta$-coloring solution for the input graph and returns the best among them.

\section{Algorithm Bipartite-3}

1: Find a $\Delta$-coloring solution for $G$;

2: Let $\langle E\rangle=\left\langle e_{1}, e_{2}, \ldots, e_{m}\right\rangle$;

3: for $p=0$ to $m-1$ do

4: $\quad$ for $q=p+1$ to $m$ do

5: $\quad$ Find, if any, a critical matching $M$ in $G\left[E_{p+1, q}\right]$;

6: $\quad$ if $M$ exists then

7: $\quad$ Find a $\left(\Delta_{1, q}-1\right)$-coloring solution for $G\left[E_{1, q} \backslash M\right]$;

8: $\quad$ else

9: $\quad$ Find a $\Delta_{1, q^{-}}$-coloring solution for $G\left[E_{1, q}\right]$;

10: $\quad$ end if

11: $\quad$ Find a $\Delta$-coloring solution for $G\left[E_{q+1, m}\right]$;

12: $\quad$ Find a solution for $G$ by concatenating the solutions found in Lines 6-10 and 11 and matching $M$, if exists;

13: end for

14: end for

15: Return the best among the solutions found in Lines 1 and 12; 
To find, if any, the critical matching $M$ in Line 5 of the algorithm, we use Proposition 3. In fact, we ask for a matching $M \subseteq E_{p+1, q}$ which saturates all the vertices of $G\left[E_{1, q}\right]$ of degree $\Delta_{1, q}$.

Theorem 9. Algorithm Bipartite-3 achieves an approximation ratio of $\frac{2(\Delta+1)^{3}}{\Delta^{3}+5 \Delta^{2}+5 \Delta+3-2(-1 / \Delta)^{\Delta}}$ for the $\mathrm{EC}(w)$ problem on bipartite graphs.

Proof. The solution obtained by a $\Delta$-coloring of the input graph computed in Line 1 of the algorithm is of weight $W_{1} \leq \Delta \cdot w_{1}^{*}$.

Consider the partition $(p, q)$ of $G$ where $w\left(e_{p+1}\right)=w_{i-1}^{*}$ and $w\left(e_{q+1}\right)=w_{i}^{*}$, for $2 \leq i \leq \Delta$ (recall that $w_{1}^{*} \geq w_{2}^{*} \geq \ldots \geq w_{k^{*}}^{*}$ and $\left.k^{*} \geq \Delta\right)$. In such an iteration, all the edges in $E_{1, q}$ belong to $i-1 \geq \Delta_{1, q}$ colors of an optimal solution $\mathcal{C}^{*}$.

If $\Delta_{1, q}<i-1$, then an $(i-2)$-coloring of $G\left[E_{1, q}\right]$ yields a solution of weight at most $(i-2) \cdot w_{1}^{*}$ for this subgraph.

If $\Delta_{1, q}=i-1$ then a critical matching $M$ exists. Indeed, in this case the $(i-1)$ th color of $\mathcal{C}^{*}$ always contains some edges from $E_{p+1, q}$, for otherwise all the edges in $E_{1, q}$ belong to $i-2$ colors of $\mathcal{C}^{*}$, a contradiction; these edges of $E_{p+1, q}$ could be a critical matching $M$ for the partition $(p, q)$. Thus, a $(i-2)$-coloring solution of $G\left[E_{1, q} \backslash M\right]$ and critical matching $M$ yield a solution for the subgraph $G\left[E_{1, q}\right]$ of weight at most $(i-2) \cdot w_{1}^{*}+w_{i-1}^{*}$. Finally, a $\Delta$-coloring solution for $G\left[E_{q+1, m}\right]$ is of cost at most $\Delta \cdot w_{i}^{*}$.

Hence, for such a partition $(p, q)$ the algorithm finds a solution for the whole input graph of weight

$$
W_{i} \leq(i-2) \cdot w_{1}^{*}+w_{i-1}^{*}+\Delta \cdot w_{i}^{*}, 2 \leq i \leq \Delta .
$$

As the algorithm returns the best among the solutions found, we have $\Delta$ bounds on the weight $W$ of this best solution, i.e.,

$$
\begin{aligned}
& W \leq \Delta \cdot w_{1}^{*}, \text { if } i=1, \text { and } \\
& W \leq(i-2) \cdot w_{1}^{*}+w_{i-1}^{*}+\Delta \cdot w_{i}^{*}, \text { if } 2 \leq i \leq \Delta
\end{aligned}
$$

To derive our ratio we perform as in the previous section, solving a system of linear equations, and we get the following multipliers:

$$
x_{i}=\left\{\begin{array}{lll}
\frac{1}{\Delta}, & \text { if } \quad i=\Delta \\
\frac{1}{\Delta+1}\left(1-\left(\frac{-1}{\Delta}\right)^{\Delta-i+1}\right), & \text { if } \quad \Delta-1 \geq i \geq 2 \\
\frac{1}{\Delta}-\sum_{j=0}^{\Delta-3}\left(\frac{\Delta-(j+2)}{\Delta} x_{\Delta-j}\right)-\frac{1}{\Delta} x_{2}, & \text { if } \quad i=1 .
\end{array}\right.
$$

By multiplying both sides of the $i$-th bound on $W$ by $x_{i}$ and adding all of them we have $\sum_{i=1}^{\Delta} x_{i} \cdot W \leq w_{1}^{*}+w_{2}^{*}+\ldots+w_{\Delta}^{*} \leq O P T$. Hence, $\frac{W}{O P T} \leq \frac{1}{\sum_{i=1}^{\Delta} x_{i}}$, which after some algebra becomes 


$$
\frac{W}{O P T} \leq \frac{(\Delta+1)}{\frac{\Delta^{3}+3 \Delta^{2}+\Delta-3}{2\left(\Delta^{2}-1\right)}-\frac{\left(\Delta^{2+(\Delta \bmod 2)}+(-1)^{\Delta}(\Delta-1)\right)}{\left(\Delta^{2}-1\right) \Delta^{\Delta}}-(\Delta-1) \sum_{i=1}^{\lfloor\Delta / 2\rfloor} \frac{2 i}{\Delta^{2 i}}} .
$$

By differentiating both sides of the formula $\sum_{i=0}^{\lfloor\Delta / 2\rfloor}\left(\frac{1}{x^{2}}\right)^{i}=\frac{1-\left(x^{-2}\right)^{\lfloor\Delta / 2\rfloor+1}}{1-x^{-2}}$ for the sum of geometric series we get

$$
-\frac{1}{x} \sum_{i=1}^{\lfloor\Delta / 2\rfloor}\left(\frac{2 i}{x^{2 i}}\right)=\frac{-2 x+2 x^{-2\lfloor\Delta / 2\rfloor+1}+2\lfloor\Delta / 2\rfloor\left(x^{2}-1\right) x^{-2\lfloor\Delta / 2\rfloor-1}}{\left(x^{2}-1\right)^{2}}
$$

and by using this last expression for $x=\Delta$ we finally get

$$
\frac{W}{O P T} \leq \frac{2(\Delta+1)^{3}}{\Delta^{3}+5 \Delta^{2}+5 \Delta+3-2(-1 / \Delta)^{\Delta}} .
$$

Lines 5-12 of the algorithm are repeated $O\left(|E|^{2}\right)$ times. Finding a critical matching in Line 5, takes, by Proposition 3, $O\left(|V|^{2.5}\right)$ time, while finding the colorings of the bipartite subgraphs of $G$ in Lines 6-10 and 11, takes $O(|E| \log \Delta)$ time [16].

\begin{tabular}{|c|c|c|c|c|}
\hline$\Delta$ & \multicolumn{2}{|c|}{ Best known ratio } & \multicolumn{2}{|r|}{ Our ratio } \\
\hline 3 & 1.17 & {$[20]$} & 1.42 & AlgORITHM BIPARTITE-1 \\
\hline 4 & 1.61 & [25] & 1.50 & ALGORITHM BIPARTITE- 1 \\
\hline 5 & 1.75 & {$[25]$} & 1.55 & Algorithm BIPARTITE- 1 \\
\hline 6 & 1.86 & {$[25]$} & 1.60 & Algorithm Bipartite- 1 \\
\hline 7 & 1.95 & {$[25]$} & 1.64 & Algorithm BIPARTITE-3 \\
\hline 8 & 2 & {$[46]$} & 1.67 & ALGORITHM BIPARTITE- 3 \\
\hline 9 & 2 & {$[46]$} & 1.69 & ALGORITHM BIPARTITE-3 \\
\hline 10 & 2 & {$[46]$} & 1.71 & Algorithm Bipartite-3 \\
\hline 11 & 2 & {$[46]$} & 1.73 & Algorithm Bipartite-3 \\
\hline 12 & 2 & {$[46]$} & 1.75 & Algorithm BIPARTITE-3 \\
\hline 13 & 2 & {$[46]$} & 1.76 & AlgORITHM BIPARTITE-3 \\
\hline 20 & 2 & [46] & 1.83 & Algorithm BIPARTITE-3 \\
\hline 50 & 2 & {$[46]$} & 1.93 & ALGORITHM BIPARTITE- 3 \\
\hline
\end{tabular}

In Table 4.2 we compare the approximation ratios achieved by our three algorithms, as $\Delta$ increases, with the best known ones.

Table 4.2: Approximation ratios for bipartite graphs

\subsection{An 1.74-approximation algorithm for bipartite graphs}

All the above algorithms for the $\mathrm{EC}(w)$ problem give good approximation guarantees for bipartite graphs of low degree. However, if the maximum degree is arbitrary large the achieved ratios tend or exceed two. In this section we exploit the same idea and 
we attain an 1.74 approximation ratio that improves substantially the known 2 approximation one for the $\mathrm{EC}(w)$ problem on bipartite graphs of maximum degree $\Delta$.

For a partition $(p, q)$ of $G$, we call a set of edges $F \subseteq E_{p+1, q}$ critical if each vertex $u \in V$ of degree $d_{1, q}(u)>\Delta_{1, p}$, has degree in $G[F]$ such that $d_{1, q}(u)-\Delta_{1, p} \leq$ $d_{G[F]}(u) \leq \Delta_{1, q}-\Delta_{1, p}$. The proposed algorithm relies on the existence of such a critical set of edges $F$ : a solution for the subgraph $G\left[E_{1, q}\right]$ is found by concatenating a $\Delta_{1, p}$-coloring solution for the subgraph $G\left[E_{1, q} \backslash F\right]$ and a $\left(\Delta_{1, q}-\Delta_{1, p}\right)$-coloring solution for the subgraph $G[F]$, if $F$ exists, and by a $\Delta_{1, q}$-coloring of the subgraph $G\left[E_{1, q}\right]$, otherwise. For each partition $(p, q)$, the algorithm computes a solution for the input graph $G$ by concatenating a solution for $G\left[E_{1, q}\right]$ and a $\Delta$-coloring solution for $G\left[E_{q+1, m}\right]$. The algorithm computes also a $\Delta$-coloring solution for the input graph and returns the best among them.

\section{Algorithm Bipartite-4}

1: Find a $\Delta$-coloring solution for $G$;

2: for $p=0$ to $m-1$ do

3: $\quad$ for $q=p+1$ to $m$ do

4: $\quad$ Find, if any, a critical set of edges $F$ in $G\left[E_{p+1, q}\right]$;

5: $\quad$ if $F$ exists then

6: $\quad$ Find a $\Delta_{1, p^{-}}$coloring solution for $G\left[E_{1, q} \backslash F\right]$;

7: $\quad$ Find a $\left(\Delta_{1, q}-\Delta_{1, p}\right)$-coloring solution for $G[F]$;

8: $\quad$ else

9: $\quad$ Find a $\Delta_{1, q^{-}}$-coloring solution for $G\left[E_{1, q}\right]$;

10: $\quad$ end if

11: $\quad$ Find a $\Delta$-coloring solution for $G\left[E_{q+1, m}\right]$;

12: $\quad$ Find a solution for $G$ by concatenating the solutions found in Lines $5-10$ and 11 ;

13: end for

14: end for

15: Return the best among the solutions found in Lines 1 and 12;

The following proposition shows that the check in Line 4 of ALGORITHM BIPARTITE4 can be done in polynomial time. This proposition is a generalization of Proposition 3 and plays a crucial role to achieve the approximation ratio of Algorithm BipARTITE- 4 .

Proposition 4. For a partition $(p, q)$ of a graph $G=(V, E)$, a critical set of edges $F$, if any, can be found in $O\left(|V|^{3}\right)$ time.

Proof. A $(g, f)$-factor of a graph $G$ is a spanning subgraph $H$ such that $g(u) \leq$ $d_{H}(u) \leq f(u)$, for all $u \in V$.

Recall that $F \subseteq E_{p+1, q}$ and consider the subgraph $G\left[E_{p+1, q}\right]$. For each vertex $u$ of $G\left[E_{p+1, q}\right]$ we define $g(u)=\max \left\{0, d_{1, q}(u)-\Delta_{1, p}\right\}$ and $f(u)=\Delta_{1, q}-\Delta_{1, p}$. Then, there exists a critical set of edges $F \subseteq E_{p+1, q}$ if and only if there exists a $(g, f)$-factor 
in $G\left[E_{p+1, q}\right]$. It is known that such a factor, if any, can be found in $O\left(|V|^{3}\right)$ time [3].

Theorem 10. Algorithm Bipartite-4 achieves an 1.74-approximation ratio for the $\mathrm{EC}(w)$ problem on bipartite graphs.

Proof. The solution obtained by a $\Delta$-coloring of the input graph computed in Line 1 of the algorithm is of weight $W_{1} \leq \Delta \cdot w_{1}^{*}$.

Consider the partition $(p, q)$ of $G$ where $w\left(e_{p+1}\right)=w_{\left\lceil\frac{i}{2}\right\rceil}^{*}$ and $w\left(e_{q+1}\right)=w_{i}^{*}$, for $2 \leq i \leq \Delta$ (recall that $w_{1}^{*} \geq w_{2}^{*} \geq \cdots \geq w_{k^{*}}^{*}$ and $k^{*} \geq \Delta$ ). In such an iteration, all the edges in $E_{1, p}$ belong to $\left\lceil\frac{i}{2}\right\rceil-1 \geq \Delta_{1, p}$ colors of an optimal solution $\mathcal{C}^{*}$, and all the edges in $E_{1, q}$ belong to $i-1 \geq \Delta_{1, q}$ colors of an optimal solution $\mathcal{C}^{*}$.

If $\Delta_{1, q}=\Delta_{1, p}$ then the set $F$ does not exist. Hence, a $\Delta_{1, q^{-}}$coloring of $G\left[E_{1, q}\right]$ yields a solution of weight at most $\left(\left\lceil\frac{i}{2}\right\rceil-1\right) \cdot w_{1}^{*}$ for this subgraph.

If $\Delta_{1, q}>\Delta_{1, p}$ then a critical set of edges $F$ exists. Indeed, in this case the colors $C_{\left\lceil\frac{i}{2}\right\rceil}^{*}, C_{\left\lceil\frac{i}{2}\right\rceil+1}^{*}, \ldots, C_{i-1}^{*}$ of $\mathcal{C}^{*}$ always contain some edges from $E_{p+1, q}$, for otherwise all the edges in $E_{1, q}$ belong to $\left\lceil\frac{i}{2}\right\rceil-1$ colors of $\mathcal{C}^{*}$, a contradiction; these edges of $E_{p+1, q}$ could be a critical set of edges $F$ for the partition $(p, q)$. Thus, a $\Delta_{1, p^{-}}$ coloring solution of $G\left[E_{1, q} \backslash F\right]$ and a $\left(\Delta_{1, q}-\Delta_{1, p}\right)$-coloring solution for $G[F]$ yield a solution for the subgraph $G\left[E_{1, q}\right]$ of weight at most $\Delta_{1, p} \cdot w_{1}^{*}+\left(\Delta_{1, q}-\Delta_{1, p}\right) \cdot w_{\left\lceil\frac{i}{2}\right\rceil}^{*} \leq$ $\left(\left\lceil\frac{i}{2}\right\rceil-1\right) \cdot w_{1}^{*}+\left\lfloor\frac{i}{2}\right\rfloor \cdot w_{\left\lceil\frac{i}{2}\right\rceil}^{*}$, since $\Delta_{1, p} \leq\left\lceil\frac{i}{2}\right\rceil-1, \Delta_{1, q} \leq i-1$ and $w_{1}^{*} \geq w_{\left\lceil\frac{i}{2}\right\rceil}^{*}$. Finally, a $\Delta$-coloring solution for $G\left[E_{q+1, m}\right]$ is of weight at most $\Delta \cdot w_{i}^{*}$.

Hence, for such a partition $(p, q)$ the algorithm finds a solution for the whole input graph of weight

$$
W_{i} \leq\left(\left\lceil\frac{i}{2}\right\rceil-1\right) \cdot w_{1}^{*}+\left\lfloor\frac{i}{2}\right\rfloor \cdot w_{\left\lceil\frac{i}{2}\right\rceil}^{*}+\Delta \cdot w_{i}^{*}, 2 \leq i \leq \Delta .
$$

As the algorithm returns the best among the solutions found, we have $\Delta$ bounds on the weight $W$ of this best solution, i.e.,

$$
\begin{aligned}
& W \leq \Delta \cdot w_{1}^{*} \text {, if } i=1, \text { and } \\
& W_{i} \leq\left(\left\lceil\frac{i}{2}\right\rceil-1\right) \cdot w_{1}^{*}+\left\lfloor\frac{i}{2}\right\rfloor \cdot w_{\left\lceil\frac{i}{2}\right\rceil}^{*}+\Delta \cdot w_{i}^{*}, \text { if } 2 \leq i \leq \Delta .
\end{aligned}
$$

To derive our ratio, we search again for appropriate multipliers, which can be found by solving a system of linear equations, as in Section 4.2. For the case where the maximum degree of the graph is a power of 2 , we get the following multipliers:

$$
x_{i}= \begin{cases}\sum_{j=0}^{\left\lfloor\log \frac{\Delta}{i}\right\rfloor}\left(-\left(\frac{-1}{\Delta}\right)^{j+1} \sum_{y=1}^{2^{j}}\left(\prod_{z=1}^{j}\left(2^{z-1}(i-1)+\left\lceil\frac{y}{2^{j-z+1}}-\frac{1}{2}\right\rceil\right)\right)\right), & \text { if } \quad \Delta \geq i \geq 2 \\ \frac{1}{\Delta}\left(1-x_{2}-\sum_{j=3}^{\Delta}\left(\left\lceil\frac{j}{2}\right\rceil-1\right) x_{j}\right), & \text { if } \quad i=1 .\end{cases}
$$

These multipliers become more complicated in the case where the maximum degree of the input graph is not a power of 2 . In this case, for $2 \leq i \leq \Delta$ we get 


$$
\begin{aligned}
& x_{i}=\sum_{j=0}^{\left\lfloor\log \frac{\Delta}{i}\right\rfloor}\left(-\left(\frac{-1}{\Delta}\right)^{j+1} \sum_{y=1}^{2^{j}}\left(\prod_{z=1}^{j}\left(2^{z-1}(i-1)+\left\lceil\frac{y}{2^{j-z+1}}-\frac{1}{2}\right\rceil\right)\right)\right)- \\
& \left(\frac{-1}{\Delta}\right)^{\left\lfloor\log \frac{\Delta}{i}\right\rfloor+2}\left(\sum _ { y = 1 } ^ { ( \Delta - i + 1 - \sum _ { r = 0 } ^ { \lfloor \operatorname { l o g } \frac { \Delta } { i } \rfloor } ( ( i - 1 ) 2 ^ { r } ) ) } \left(\prod _ { z = 1 } ^ { \lfloor \operatorname { l o g } \frac { \Delta } { i } \rfloor + 1 } \left(2^{z-1}(i-1)+\left\lceil\frac{y}{\left.\left.\left.2^{\left\lfloor\log \frac{\Delta}{i}\right\rfloor+2-z}-\frac{1}{2}\right\rceil\right)\right)}\right.\right.\right.\right.
\end{aligned}
$$

while $x_{1}$ is the same as in the previous case.

By multiplying both sides of the $i$-th bound on $W$ by $x_{i}$ and adding all of them we have $\sum_{i=1}^{\Delta} x_{i} \cdot W \leq w_{1}^{*}+w_{2}^{*}+\ldots+w_{\Delta}^{*} \leq O P T$, and hence, $\frac{W}{O P T} \leq \frac{1}{\sum_{i=1}^{\Delta} x_{i}}$.

Using Mathematica, we compute the above ratio for quite large values of $\Delta$, and it is found to tend to 1.74 . Table 4.3 shows the values of the ratio as $\Delta$ increases. However, an interesting question is whether we can compute a close formula for this ratio.

\begin{tabular}{c|c}
\hline \hline$\Delta$ & Our ratio \\
\hline \hline $2^{2}$ & 1.48837 \\
$2^{3}$ & 1.60188 \\
$2^{4}$ & 1.66582 \\
$2^{5}$ & 1.70023 \\
$2^{6}$ & 1.71809 \\
$2^{7}$ & 1.72719 \\
$2^{8}$ & 1.73178 \\
$2^{9}$ & 1.73409 \\
$2^{10}$ & 1.73525 \\
$2^{11}$ & 1.73583 \\
$2^{12}$ & 1.73612 \\
$2^{13}$ & 1.73626 \\
$2^{14}$ & 1.73634 \\
$2^{15}$ & 1.73637 \\
$2^{16}$ & 1.73639 \\
$2^{17}$ & 1.73640 \\
\hline \hline
\end{tabular}

Table 4.3: Approximation ratios achieved by Algorithm Bipartite-4 for different values of $\Delta$.

\subsection{Bi-valued graphs}

In this section we show first that the $\mathrm{EC}(w)$ problem is NP-complete for complete graphs with bi-valued edge weights. Recall that the $\mathrm{EC}(w)$ problem is polynomial for bi-valued bipartite graphs [22], while for general bi-valued graphs it generalizes the classical edge-coloring problem, which is known to be NP-complete even for cubic graphs [41]. In the next theorem we give a reduction from this latter problem. 
Theorem 11. The $\mathrm{EC}(w)$ problem is NP-complete for complete graphs even with edge weights $w(e) \in\{1,2\}$.

Proof. The edge-coloring problem for cubic graphs takes as input a graph $G=$ $(V, E),|V|=n$, with $d(u)=3$, for each $u \in V$, and asks for the existence of a proper 3-coloring of $G$. Notice that any cubic graph has an even number, $n$, of vertices.

From such an instance we construct a complete weighted graph $K_{n}$ with edge weights $w(e)=2$, for each $e \in E$, and $w(e)=1$, otherwise, and we show that there is a 3 -coloring of $G$ iff there is a solution $\mathcal{C}$ for the $\operatorname{EC}(w)$ problem on $K_{n}$ of weight at most $n+2$.

Assume, first, that there is a 3-coloring of $G$. Then, there are three colors of $K_{n}$ each one of weight equal to 2 , which include all the edges of $K_{n}$ of weight 2 . Let $K_{n}-G$ be the graph induced by the remaining edges of $K_{n}$ (those of weight 1 ). The graph $K_{n}-G$ is $(n-4)$-colorable as a $(n-4)$-regular graph of even order [13]. Therefore, there is a solution $\mathcal{C}$ for the $\operatorname{EC}(w)$ problem on $K_{n}$ of weight at most $3 \cdot 2+(n-4) \cdot 1=n+2$.

Conversely, consider that there is a solution $\mathcal{C}$ to the $\mathrm{EC}(w)$ problem on $K_{n}$ of weight at most $n+2$. This solution contains $k \geq n-1$ colors, since a complete graph of even order can be colored with at least $n-1$ colors [27]. Moreover, $\mathcal{C}$ contains at least three colors of weight equal to 2 , since, by its construction, $K_{n}$ has exactly three edges of weight 2 adjacent to each vertex. Assume that there is a fourth color in $\mathcal{C}$ of weight equal to 2 . Then, $\mathcal{C}$ will be of weight at least $4 \cdot 2+(k-4) \cdot 1 \geq n+3$, a contradiction. Therefore, $\mathcal{C}$ contains exactly 3 colors of weight equal to 2 , which imply a 3 -coloring for $G$.

Theorem 11 implies that the $\mathrm{EC}(w)$ problem is NP-complete in all superclasses of complete graphs, including split and interval graphs. Note also that the complexity of the classical edge-coloring problem on interval graphs of even maximum degree remains an open question [7]. Next corollary follows also from Theorem 11.

Corollary 1. It is NP-hard to approximate the $\mathrm{EC}(w)$ problem within a ratio less than $\frac{n+3}{n+2}$ in complete graphs.

In what follows, we present an approximation algorithm for general graphs with two different edge weights. Assume that the edges of the graph $G=(V, E)$ have weights either 1 or $t \geq 2$. Let $G\left[E_{1}\right]$, of maximum degree $\Delta_{1}$, and $G\left[E_{t}\right]$, of maximum degree $\Delta_{t}$, be the subgraphs of $G$ induced by its edges of weights 1 and $t$, respectively.

\section{Algorithm Bi-VAlued}

1: Find a $(\Delta+1)$-coloring solution for $G$;

2: Find a $\left(\Delta_{1}+1\right)$-coloring solution for $G\left[E_{1}\right]$, a $\left(\Delta_{t}+1\right)$-coloring solution for $G\left[E_{t}\right]$ and concatenate them;

3: Return the best among the two solutions found in Lines 1 and 2; 
Theorem 12. Algorithm BI-VAlued achieves a $\frac{4}{3}$-approximation ratio for the $\mathrm{EC}(w)$ problem on general graphs of arbitrarily large $\Delta$ and edge weights $w(e) \in$ $\{1, t\}$.

Proof. An optimal solution contains at least $\Delta$ colors and at least $\Delta_{t}$ of them are of weight equal to $t$. Therefore, a lower bound to the weight of an optimal solution is $O P T \geq \Delta_{t} \cdot t+\left(\Delta-\Delta_{t}\right)$.

A $(\Delta+1)$-coloring of $G$ in Line 1 of the algorithm yields a solution for the $\mathrm{EC}(w)$ problem of weight $W \leq(\Delta+1) \cdot t$, while a $\left(\Delta_{1}+1\right)$-coloring of $G\left[E_{1}\right]$ and a $\left(\Delta_{t}+1\right)$-coloring of $G\left[E_{t}\right]$ in Line 2 yield another solution of weight $W \leq$ $\left(\Delta_{t}+1\right) \cdot t+\left(\Delta_{1}+1\right) \cdot 1 \leq\left(\Delta_{t}+1\right) \cdot t+(\Delta+1)$. By multiplying both sides of the first inequality with $\frac{\Delta_{t}^{2}+2 \Delta_{t}-\Delta}{(\Delta+1)^{2}}$, both sides of the second one with $\frac{\Delta-\Delta_{t}}{\Delta+1}$ and adding them, we get $\frac{\Delta^{2}+\Delta_{t}^{2}-\Delta \cdot \Delta_{t}+\Delta_{t}}{(\Delta+1)^{2}} \cdot W \leq \Delta_{t} \cdot t+\left(\Delta-\Delta_{t}\right) \leq O P T$, that is $\frac{W}{O P T} \leq \frac{(\Delta+1)^{2}}{\left(\Delta-\Delta_{t}\right)^{2}+\Delta_{t}(\Delta+1)}$. This ratio is maximized when $\Delta_{t}=\frac{\Delta-1}{2}$, and therefore $\frac{W}{O P T} \leq \frac{4(\Delta+1)}{(\Delta+1)+2(\Delta-1)}=\frac{4 \Delta+4}{3 \Delta-1}=\frac{4}{3}+\frac{16}{9 \Delta-3}$. 


\section{Chapter 5}

\section{Max-Edge-Coloring on trees}

In this chapter we describe approximation results for the $\mathrm{EC}(w)$ problem on trees, as well as polynomial algorithms for subclasses of trees.

In Section 5.1 we present a polynomial algorithm for a special class of trees, namely stars of chains (or spiders). To obtain this algorithm we need the following theorem for trees of bounded maximum degree.

Theorem 13. The $\mathrm{EC}(w)$ problem can be solved in polynomial time on trees of bounded maximum degree.

Proof. By Theorem 2(i), the $\mathrm{EC}(w)$ problem is polynomial on trees when the number, $k$, of colors is fixed. Moreover, by Proposition 2 it holds that $k \leq 2 \Delta-1$, and hence the theorem follows.

Although the complexity question for the $\mathrm{EC}(w)$ problem on trees remains open, we give, in Section 5.2, a 3/2-approximation algorithm. Next, we present two approximation algorithms of low exponential complexity. The complexity of the first algorithm is exponential to the maximum degree of the tree, while the second one is exponential to the number of edges. Finally, a PTAS is presented for the $\mathrm{EC}(w)$ problem on trees in Section 5.4, reaching the approximability of the $\mathrm{VC}(w)$ problem on the same class of graphs.

\subsection{Stars of chains}

A star consists of $m$ edges, $e_{1}, e_{2}, \ldots, e_{m}$, sharing a common endpoint. Obviously, the optimal solution to the $\operatorname{EC}(w)$ problem for such a weighted star is of weight $O P T=\sum_{j=1}^{m} w\left(e_{j}\right)$ and consists of exactly $\Delta=m$ colors.

A star of chains consists of $\Delta \geq 3$ chains, $T_{1}, T_{2}, \ldots, T_{\Delta}$, all starting from a common vertex, say $u$. We consider each chain $T_{j}, 1 \leq j \leq \Delta$, starting from $u$ with an edge $e_{j}^{u}$ which we call start edge. We also assume, w.l.o.g., that $w\left(e_{1}^{u}\right) \geq w\left(e_{2}^{u}\right) \geq$ $\ldots \geq w\left(e_{\Delta}^{u}\right)$.

Lemma 3. For an optimal solution $\mathcal{C}^{*}=\left\{C_{1}^{*}, C_{2}^{*}, \ldots, C_{k^{*}}^{*}\right\}$ of the $\mathrm{EC}(w)$ problem on a star of chains the following hold: 
(i) The number of colors $k^{*}$ equals to either $\Delta$ or $\Delta+1$.

(ii) Only $p \leq 3$ colors have cardinality $\left|C_{i}^{*}\right|>1$.

(iii) At least the $p-1$ heaviest start edges appear in these $p$ colors.

Proof. For item (i), according to Proposition $2, \Delta \leq k^{*} \leq \Delta^{\prime}-1$. Here, it holds that $\Delta^{\prime}=\Delta+2$.

In order to prove item (ii), assume that an optimal solution has more than three colors of cardinality $\left|C_{i}^{*}\right|>1$. Consider those colors sorted in non-increasing order with respect to their weights. Each non start edge $e$ has at most two neighbor edges. So, such an edge $e$ can be moved in one of the three first heaviest colors, since its neighbor edges can belong in at most two different colors.

Finally, for item (iii), consider first that $p=2$. Assume that in the optimal solution the heaviest start edge $e_{1}^{u}$ does not belong to neither of two colors of cardinality $\left|C_{i}^{*}\right|>1$. Then $e_{1}^{u}$ can be either inserted into one of those two colors (if this does not contain another start edge), or $e_{1}^{u}$ can replace an existing start edge. In both cases the weight of the optimal solution does not increase.

Assume next that $p=3$. As in the previous case, $e_{1}^{u}$ can be inserted into one of the three colors of cardinality $\left|C_{i}^{*}\right|>1$. Similarly, $e_{2}^{u}$ can be inserted in one of the remaining two of those colors.

In what follows, we distinguish between two cases according to possible number of colors in an optimal solution, i.e., $\Delta+1$ or $\Delta$.

If an optimal solution consists of $\Delta+1$ colors, then it contains exactly one color without any start edge. Algorithm Star-of-Chains $(\Delta+1)$ finds such an optimal solution with $\Delta+1$ colors.

\author{
Algorithm Star-of-Chains $(\Delta+1)$ \\ 1: Remove from the star the $\Delta-2$ lightest start edges; \\ (this creates a graph $H$ consisting of $\Delta-1$ chains) \\ 2: Find an optimal solution $S^{*}(H)$ for the graph $H$, using Theorem 6; \\ 3: if there are 3 non empty colors in $S^{*}(H)$ then \\ 4: Return the solution consisting of these 3 colors of $S^{*}(H)$ plus $\Delta-2$ colors \\ each one containing one of the removed $\Delta-2$ lightest start edges; \\ 5: end if
}

Note that Algorithm $\operatorname{StaR-OF-Chains~}(\Delta+1)$ it is possible to return $\Delta-1$ colors of $\left|C_{i}\right|=1$, in the case where one of the three colors of $S^{*}(H)$ found in Line 2 consists of a single edge. Taking into account Lemma 3, it follows that Algorithm StaR-OF-Chains $(\Delta+1)$ returns an optimal solution of $\Delta+1$ colors since: (i) the $\Delta-(p-1)$ colors of cardinality $\left|C_{i}\right|=1$ contain the $\Delta-(p-1)$ lightest start edges (one per each color), and (ii) the weight of $p$ colors is optimal.

The complexity of Algorithm $\operatorname{StaR-OF-Chains}(\Delta+1)$ is dominated by Line 2 and by Theorem 6 it is $O(|E| \log |E|)$. 
If an optimal solution consists of $\Delta$ colors, then each of them contains a start edge. Algorithm Star-OF-Chains $(\Delta)$ returns such an optimal solution with $\Delta$ colors.

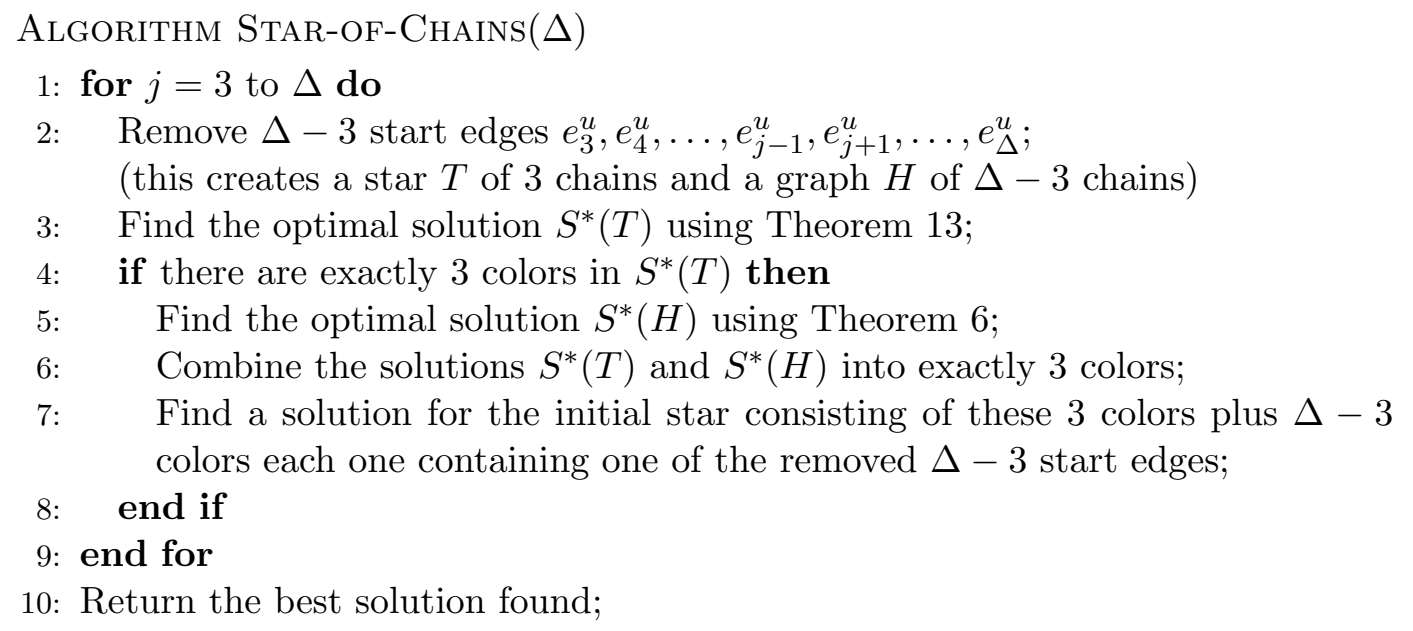

The star $T$, obtained after Line 2 of the above algorithm, is a tree of maximum degree three and therefore an optimal solution $S^{*}(T)$, in Line 3, can be found in polynomial time, by Theorem 13. Notice that such a solution consists of at least three colors. Moreover, the optimal solution $S^{*}(H)$ built in Line 5, consists of at most three colors, by Proposition 2.

In Line 6, an optimal solution of three colors for the edges in $T$ and $H$ can be obtained by considering the colors in both solutions in non-increasing order with respect to their weights and merging the colors of each solution having the same rank, since $T$ and $H$ are vertex-disjoint. The optimality of the solution that ALGORITHM $\operatorname{StaR-OF-Chains}(\Delta)$ returns, follows from Lemma 3 using the same arguments as for Algorithm Star-of-Chains $(\Delta+1)$.

The complexity of the algorithm is dominated by Line 3 which takes polynomial time and is executed $\Delta-2$ times.

By Lemma 3 the optimal solution to the $\mathrm{EC}(w)$ problem on stars of chains is the best between the solution found by Algorithm Star-OF-Chains $(\Delta+1)$ (optimal with $\Delta+1$ colors) and the one found by Algorithm Star-of-Chains $(\Delta)$ (optimal with $\Delta$ colors). Thus, the following theorem holds.

Theorem 14. The $\mathrm{EC}(w)$ problem is polynomial on stars of chains.

\subsection{A 3/2 approximation algorithm}

In this section, we first present an $\left(1+\frac{w_{1}^{*}-w_{\Delta}^{*}}{O P T}\right)$-approximation algorithm for the $\mathrm{EC}(w)$ problem on trees. Then, combining this algorithm with Algorithm KK we derive a $3 / 2$ approximation ratio. 
For our first algorithm we consider the tree rooted in an arbitrary vertex and we denote by $E^{u}$ the edges of the tree adjacent to a vertex $u$. The algorithm traverses the vertices of the tree in pre-order and for each vertex $u$ assigns the edges in $E^{u}$ to colors as follows.

\section{Algorithm Trees}

1: Root the tree in an arbitrary vertex $r$;

2: for each vertex $u$ in a pre-order traversal of the tree do

3: $\quad$ Let $\left\langle E^{u}\right\rangle=\left\langle e_{1}^{u}, e_{2}^{u}, \ldots, e_{d(u)}^{u}\right\rangle$, and $e_{j}^{u}, 1 \leq j \leq d(u)$, be the edge between $u, u \neq r$, and its parent;

4: $\quad$ for $i=1$ to $d(u), i \neq j$, do

5: $\quad$ Insert edge $e_{i}^{u}$ into the first color not containing other edge in $E^{u}$;

6: $\quad$ end for

7: end for

To analyze our algorithm we define $y_{i}, 1 \leq i \leq \Delta$, to be the weight of the heaviest edge between those ranked $i$ in each ordering $\left\langle E^{u}\right\rangle, u \in V$, i.e., $y_{i}=\max _{u \in V}\left\{w\left(e_{i}^{u}\right)\right\}$. It is clear that $y_{1} \geq y_{2} \geq \ldots \geq y_{\Delta}$. Next two propositions use these values for bounding the weights of the colors of both an optimal solution and a solution found by Algorithm Trees. Recall that an optimal solution to the $\mathrm{EC}(w)$ problem consists of at least $\Delta$ colors.

Proposition 5. For all $1 \leq i \leq \Delta$, it holds that $w_{i}^{*} \geq y_{i}$.

Proof. Let $e=(u, v)$ be the heaviest edge with rank equal to $i$, i.e., $y_{i}=w(e)$. W.l.o.g., assume that $e$ is ranked $i$ in $E^{u}$. Then, there exist $i$ edges in $E^{u}$ of weight at least $y_{i}$ and as they belong into $i$ different colors in an optimal solution, it follows that $w_{i}^{*} \geq y_{i}$.

Proposition 6. Algorithm Trees constructs a solution of exactly $\Delta$ colors. For the weight, $w_{i}$, of the $i$-th, $2 \leq i \leq \Delta$, color it holds that $w_{i} \leq y_{i-1}$.

Proof. For a vertex $u \neq r$ of the tree let $e$ be the edge between $u$ and its parent and $j$ be its rank in $E^{u}$, i.e., $e=e_{j}^{u}$. In the iteration processing the vertex $u$ the edge $e$ has already been inserted by the algorithm into a color, say $C_{p}$.

The algorithm inserts the edges in $E^{r}$ into $d(r) \leq \Delta$ colors. For any other vertex $u$, the algorithm inserts the edges in $E^{u} \backslash\{e\}$ into $d(u)-1 \leq \Delta-1$ colors different than $C_{p}$. Therefore, the algorithm finds a solution $\mathcal{C}=\left\{C_{1}, C_{2}, \ldots, C_{\Delta}\right\}$ of exactly $\Delta$ colors.

We prove the bounds on the color's weights by induction on the vertices in the order they are processed by the algorithm. We consider all colors in $\mathcal{C}$ of an initial weight $w_{i}=0,1 \leq i \leq \Delta$.

For the root vertex $r$, the algorithm inserts each edge $e_{i}^{r}$ into color $C_{i}, 1 \leq i \leq$ $d(r)$. Clearly, $w_{i}=w\left(e_{i}^{r}\right) \leq y_{i} \leq y_{i-1}, 2 \leq i \leq \Delta$.

Assume that before the iteration processing a vertex $u \neq r$, it holds that $w_{i} \leq$ $y_{i-1}, 2 \leq i \leq \Delta$, and let $w_{i}^{\prime}$ be the weight of the color $C_{i}, 2 \leq i \leq \Delta$, after processing 
the vertex $u$. We prove that $w_{i}^{\prime} \leq y_{i-1}, 2 \leq i \leq \Delta$, by distinguishing among three cases depending on the values of $p$ and $j$ :

(i) $p=j$ : Each edge $e_{i}^{u}$ belongs to color $C_{i}, 1 \leq i \leq d(u)$. Since $w_{i} \leq y_{i-1}$ and $w\left(e_{i}^{u}\right) \leq y_{i}$, it follows that $w_{i}^{\prime}=\max \left\{w_{i}, w\left(e_{i}^{u}\right)\right\} \leq \max \left\{y_{i-1}, y_{i}\right\}=y_{i-1}$, $2 \leq i \leq \Delta$.

(ii) $p>j$ : For $1 \leq i \leq j-1$ and $p+1 \leq i \leq d(u)$ each edge $e_{i}^{u}$ belongs to color $C_{i}$ and we conclude as in Case (i). For $j+1 \leq i \leq p$ each edge $e_{i}^{u}$ belongs to color $C_{i-1}$, that is $w_{i}^{\prime}=\max \left\{w_{i}, w\left(e_{i+1}^{u}\right)\right\} \leq \max \left\{y_{i-1}, y_{i+1}\right\}=y_{i-1}$.

(iii) $p<j$ : For $1 \leq i \leq p-1$ and $j+1 \leq i \leq d(u)$ each edge $e_{i}^{u}$ belongs to color $C_{i}$ and we conclude as in Case (i). For $p \leq i \leq j-1$ each edge $e_{i}^{v}$ belongs to color $C_{i+1}$, that is $w_{i}^{\prime}=\max \left\{w_{i}, w\left(e_{i-1}^{u}\right)\right\} \leq \max \left\{y_{i-1}, y_{i-1}\right\}=y_{i-1}$.

Using the bounds established in Propositions 5 and 6 we obtain the next lemma.

Lemma 4. Algorithm TRees achieves an approximation ratio of $1+\frac{w_{1}^{*}-w_{\Delta}^{*}}{O P T}<2$ for the $\mathrm{EC}(w)$ problem on trees.

Proof. For the weight of the first color obtained by ALgorithm TreEs it holds that $w_{1} \leq y_{1}=w_{1}^{*}$, since both $y_{1}$ and $w_{1}^{*}$ are equal to the weight of the heaviest edge of the tree. By Proposition 6 it holds that $w_{i} \leq y_{i-1}, 2 \leq i \leq \Delta$ and by Proposition 5 it holds that $y_{i} \leq w_{i}^{*}, 1 \leq i \leq \Delta$. Therefore, the weight of the solution obtained by Algorithm Trees is $W=\sum_{i=1}^{\Delta} w_{i} \leq y_{1}+\sum_{i=2}^{\Delta} y_{i-1}=y_{1}+\sum_{i=1}^{\Delta-1} y_{i} \leq w_{1}^{*}+\sum_{i=1}^{\Delta-1} w_{i}^{*} \leq$ $w_{1}^{*}+O P T-w_{\Delta}^{*}$, that is $\frac{W}{O P T} \leq 1+\frac{w_{1}^{*}-w_{\Delta}^{*}}{O P T}<2$.

The example illustrated in Figure 5.1(a) shows that the ratio of our algorithm can be arbitrarily close to 2 . For this instance $O P T=1+2 \epsilon$ (Figure 5.1(b)), the weight of the solution found by Algorithm Trees is $W=2+\epsilon$ (Figure 5.1(c)) and the approximation ratio becomes $\frac{2+\epsilon}{1+2 \epsilon}$.

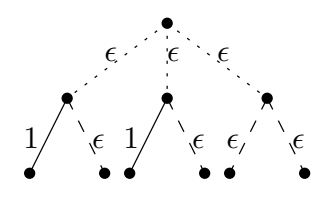

$(a)$

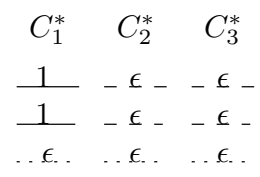

(b)

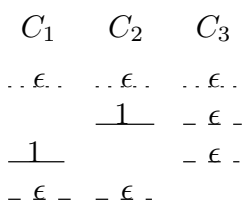

(c)

Figure 5.1: A tight example for the 2 approximation ratio of Algorithm Trees $(\Delta=3, \epsilon<<1)$. 
To derive the $3 / 2$ approximation ratio we simply select the best among the solutions found by Algorithm KK and Algorithm Trees.

Theorem 15. There is a $\frac{3}{2}$-approximation algorithm for the $\mathrm{EC}(w)$ problem on trees.

Proof. Let $W$ be the weight of the best among the solutions found by AlGORITHM KK and Algorithm Trees. By Lemma 1 it holds that $\frac{W}{O P T} \leq 2-\frac{w_{1}^{*}}{O P T}$ and by Lemma 4 that $\frac{W}{O P T} \leq 1+\frac{w_{1}^{*}-w_{\Delta}^{*}}{O P T}$. As the first bound is increasing and the second one is decreasing with respect to $O P T$, it follows that the ratio $\frac{W}{O P T}$ is maximized when $2-\frac{w_{1}^{*}}{O P T}=1+\frac{w_{1}^{*}-w_{\Delta}^{*}}{O P T}$, that is $O P T=2 \cdot w_{1}^{*}-w_{\Delta}^{*}$. Therefore, $\frac{W}{O P T} \leq 2-\frac{w_{1}^{*}}{O P T}=2-\frac{w_{1}^{*}}{2 \cdot w_{1}^{*}-w_{\Delta}^{*}} \leq 2-\frac{w_{1}^{*}}{2 \cdot w_{1}^{*}}=\frac{3}{2}$.

For the tightness of the analysis in Theorem 15 consider the instance given in Figure 5.2(a). For this instance $O P T=2+2 \epsilon$ (Figure 5.2(b)) and the weights of the solutions found by Algorithm Trees and Algorithm KK are 3 (Figure 5.2(c)) and $3-\epsilon$ (Figure 5.2(d)), respectively. Our algorithm selects the solution found by Algorithm KK and the approximation ratio becomes $\frac{3-\epsilon}{2+2 \epsilon}$.

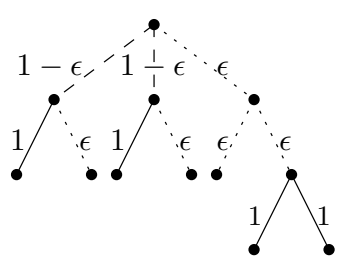

(a)

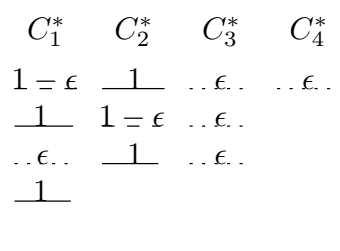

(b)

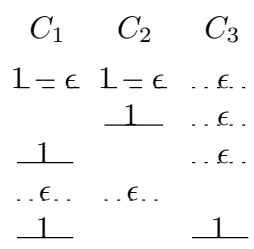

(c)

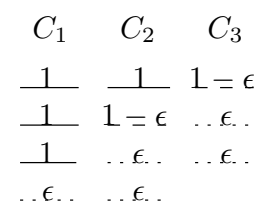

$(d)$

Figure 5.2: A tight example for the 3/2-approximation algorithm for trees $(\Delta=3$, $\epsilon<<1)$.

\subsection{Moderately exponential approximation algorithms}

In this section, we present two approximation algorithms for trees that improve the $3 / 2$ ratio of Theorem 15 within exponential running time much better than that needed for the computation of an optimal solution.

The idea employed by the algorithms is to find an approximate solution to the $\mathrm{EC}(w)$ problem on a tree $T=(V, E)$ by searching exhaustively for the weights of a number of colors of an optimal solution $\mathcal{C}^{*}$. A parameter $z$, given as input to the algorithms, determines the (maximum) number of colors of $\mathcal{C}^{*}$ that we search 
exhaustively and, hence, the complexity and the approximation ratio of the algorithms. In such an exhaustive search, each step of the proposed algorithms has to answer to an instance of the FEASIBLE-EC $(w)$ problem, which can be done through the $\operatorname{EC}(\phi)$ problem (see Section 3.1).

The first algorithm proposed in Section 5.3 is exponential to the maximum degree, $\Delta$, of the input tree and achieves a $\rho$ approximation ratio in $O^{*}\left(m^{f(\rho) \cdot \Delta}\right)$ time, where $f(\rho)=\frac{9-\rho}{4 \rho}$. The second algorithm presented in Section 5.3 is exponential to the number of edges, $m$, of the input tree and achieves a ratio of $\rho$ in $O^{*}\left(g(\rho)^{m}\right)$ time, where $g(\rho)=\frac{(2 \rho-1)^{2}+1}{(2 \rho-1)^{2(2 \rho-1)^{2} /\left((2 \rho-1)^{2}+1\right)}}$. Some values of $\rho \leq 3 / 2, f(\rho)$ and $g(\rho)$ are summarized in Table 5.1.

\begin{tabular}{l||c|c|c|c|c|c|c} 
Complexity & $\rho$ & $O P T$ & 1.1 & 1.2 & 1.3 & 1.4 & 1.5 \\
\hline$O^{*}\left(m^{f(\rho) \cdot \Delta}\right)$ & $f(\rho)$ & 2 & 1.795 & 1.625 & 1.481 & 1.357 & 1.250 \\
$O^{*}\left(g(\rho)^{m}\right)$ & $g(\rho)$ & 2 & 1.968 & 1.896 & 1.811 & 1.727 & 1.649
\end{tabular}

Table 5.1: Approximation ratios vs. complexities for trees

\section{An exponential to $\Delta$ algorithm}

This algorithm depends on a parameter $z$ taking integer values in $[1,2 \Delta-1]$ and iterates $z$ times, for $j=1,2, \ldots z$. In each iteration the algorithm considers all the combinations of $j$ edge weights as the weights of the $j$ heaviest colors of an optimal solution. For each combination of weights, $w_{1} \geq w_{2} \geq \ldots \geq w_{j}$, the algorithm has to answer to an instance of the FEASIBLE- $\mathrm{EC}(w)$ problem on the input tree $T$. In order a "yes" answer to this FeAsible-EC $(w)$ problem to be probable for all values of $j$ we extend the combination of weights $w_{1} \geq w_{2} \geq \ldots \geq w_{j}$ to a sequence $w_{1} \geq w_{2} \geq \ldots \geq w_{j}=w_{j+1}=w_{j+2}=\ldots=w_{k}$ by adding $k-j$ new weights all equal to $w_{j}$. In fact, this extended sequence consists of $k=j-1+\Delta$ weights, if $j \leq \Delta$ (this way the $T$ 's edges of weights $w(e) \leq w_{j}$ can be assigned into the $\Delta$ colors of weight $w_{j}$ ) and $k=2 \Delta-1$, otherwise (since by Proposition 2 any solution to the $\operatorname{EC}(w)$ problem consists of at most $2 \Delta-1$ colors). Hence, $k=\min \{j-1+\Delta, 2 \Delta-1\}$. This instance of the FEAsible-EC $(w)$ problem has answer "yes" if and only if the edges of weight $w(e)>w_{j}$ can be assigned to (colors of) weights greater than $w_{j}$ (see the proof of Theorem 16). In this case the algorithm finds a feasible solution for the $\operatorname{EC}(w)$ problem and it returns the best among all feasible solutions found. 


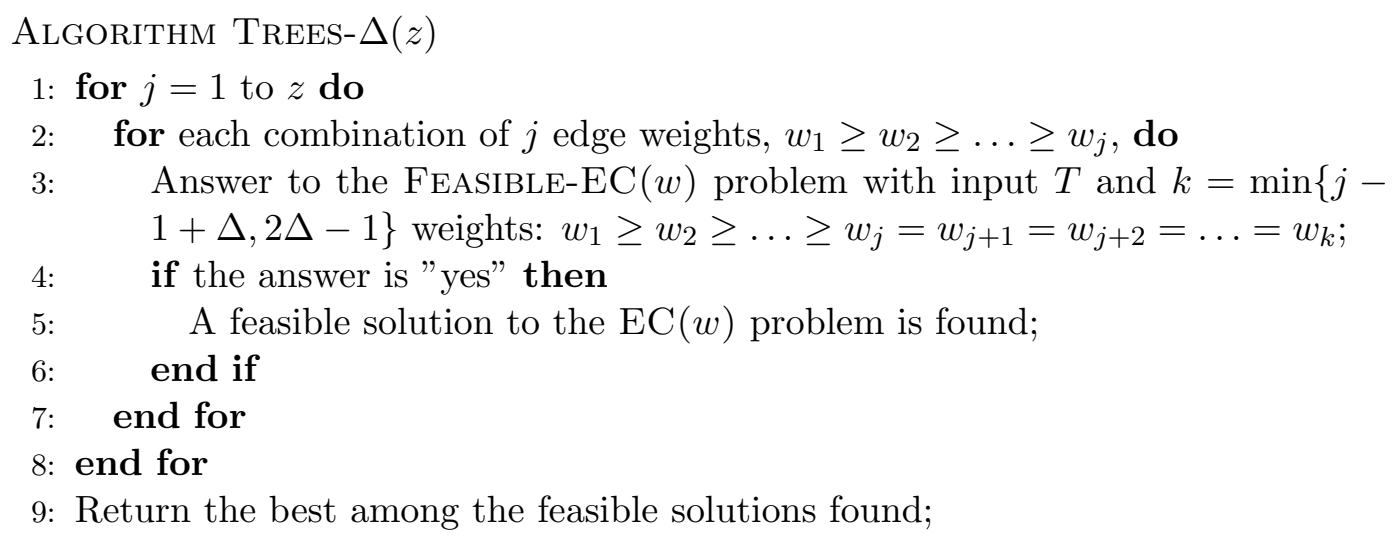

Theorem 16. For any $\rho \geq 1$, Algorithm Trees- $\Delta(z)$ achieves a $\rho$ approximation ratio for the $\mathrm{EC}(w)$ problem on trees, in polynomial space and running time $O^{*}\left(m^{f(\rho) \Delta}\right)$, where $f(\rho)=\frac{9-\rho}{4 \rho}$.

Proof. Consider the $j$-th iteration of the algorithm and in this iteration the combination of $j$ edge weights which coincide with the weights $w_{1}^{*} \geq w_{2}^{*} \geq \ldots \geq w_{j}^{*}$, of the $j$ heaviest colors of an optimal solution $\mathcal{C}^{*}$. In this step the algorithm answers to the instance of the FEasible-EC $(w)$ problem with input $T$ and weights $w_{i} \geq w_{i}^{*}$, $1 \leq i \leq k$. We claim that this FeASIBLE-EC $(w)$ problem has always a "yes" answer. Indeed, if $k=2 \Delta-1$, then the claim follows since $k^{*} \leq 2 \Delta-1$ and $w_{i} \geq w_{i}^{*}$, $1 \leq i \leq k^{*}$. If $k=j-1+\Delta<2 \Delta-1$, then the edges of weights $w(e)>w_{j}^{*}$ can be assigned (belong) to the $j-1$ heaviest weights (colors of $\mathcal{C}^{*}$ ). Moreover, there are $\Delta$ weights equal to $w_{j}^{*}$ and the edges of weights $w(e) \leq w_{j}^{*}$ can be assigned to them. Hence, a feasible solution for the $\mathrm{EC}(w)$ problem on $T$ is found of weight

$$
W_{j}=w_{1}^{*}+w_{2}^{*}+\ldots+w_{j-1}^{*}+(k-j+1) \cdot w_{j}^{*} .
$$

The algorithm finds such a feasible solution in each iteration $j$ and as it returns the best among them we obtain $\Delta$ bounds on the weight of this best solution, that is $W \leq w_{1}^{*}+w_{2}^{*}+\ldots+w_{j-1}^{*}+(k-j+1) \cdot w_{j}^{*}, 1 \leq j \leq z$. Proceeding as in the proof of Theorem 8 we find $z$ multipliers

$$
x_{j}=\left\{\begin{array}{lll}
\frac{2 \Delta-1-z}{\Delta^{2}}\left(\frac{\Delta-1}{\Delta}\right)^{\Delta-1-j}, & \text { if } & 1 \leq j \leq \Delta \\
\frac{2 \Delta-1-z}{(2 \Delta-j)(2 \Delta-1-j)}, & \text { if } & \Delta+1 \leq j \leq z
\end{array}\right.
$$

such that $\frac{W}{O P T} \leq \frac{1}{\sum_{i=1}^{z} x_{i}}=\frac{1}{1-\frac{2 \Delta-1-z}{\Delta} \cdot\left(\frac{\Delta-1}{\Delta}\right)^{\Delta-1}}$.

The $\operatorname{EC}(w)$ problem is polynomial for graphs of $\Delta=2$ and as for $\Delta \geq 3$ it holds that $\left(\frac{\Delta-1}{\Delta}\right)^{\Delta-1}>\frac{4}{9}$ we get $\frac{W}{O P T} \leq \frac{1}{1-\frac{4}{9} \cdot \frac{2 \Delta-1-z}{\Delta}}=\rho$. Hence, an approximation ratio $\rho$ is derived for $z=\frac{9-\rho}{4 \rho} \cdot \Delta-1=f(\rho) \cdot \Delta-1$, where $f(\rho)=\frac{9-\rho}{4 \rho}$. 
The complexity of Algorithm Trees- $\Delta(z)$ is exponential in $z$. In Line 2 the algorithm examines $\left(\begin{array}{c}m \\ j\end{array}\right)$ combinations of weights. Thus, for all iterations $\sum_{j=1}^{z}\left(\begin{array}{c}m \\ j\end{array}\right)=$ $O\left(z \cdot m^{z}\right)$ combinations of weights are examined. For each one of these combinations, it takes $O\left(m \cdot \Delta^{3.5}\right)$ time to answer to the instance of the Feasible-EC $(w)$ in Line 3. Since $z$ and $\Delta$ are $O(m)$, the complexity of Algorithm Trees- $\Delta(z)$ is $O^{*}\left(m^{z}\right)$, that is $O^{*}\left(m^{f(\rho) \Delta}\right)$. Moreover, the algorithm needs polynomial space, since Line 3 is executed independently for each combination of weights.

Notice that for $z=2 \Delta-1$ the Algorithm Trees- $\Delta(z)$ finds an optimal solution within $O^{*}\left(m^{2 \Delta}\right)$ time.

\section{An exponential to $m$ algorithm}

This algorithm depends on a parameter $z$ taking integer values in $\left[1,\left\lfloor\frac{m}{2}\right\rfloor\right]$ and iterates $2 z$ times, for $k=1,2, \ldots, z, m-z, \ldots, m$. In each iteration, the algorithm exhaustively considers $k$ edge weights, $w_{1}, w_{2}, \ldots, w_{k}$, as the weights of the $k$ heaviest colors of an optimal solution $\mathcal{C}^{*}$, and answers to the instance of the FEAsiBLE$\operatorname{EC}(w)$ problem, with input $T$ and $w_{1} \geq w_{2} \geq \ldots \geq w_{k}$. This way an optimal solution is found when $k^{*} \leq z$ or $k^{*} \geq m-z$. In order to derive an approximate solution when $z<k^{*}<m-z$, the algorithm, in the iteration where $k=z$, answers also to instances of the FEASIBLE-EC $(w)$ problem with input $T$ and weights $w_{1} \geq w_{2} \geq \ldots \geq w_{z}=w_{z+1}=\ldots=w_{k^{\prime}}$, for $k^{\prime}=z+1, z+2, \ldots, m-z-1$. The algorithm returns the best among the feasible solutions found.

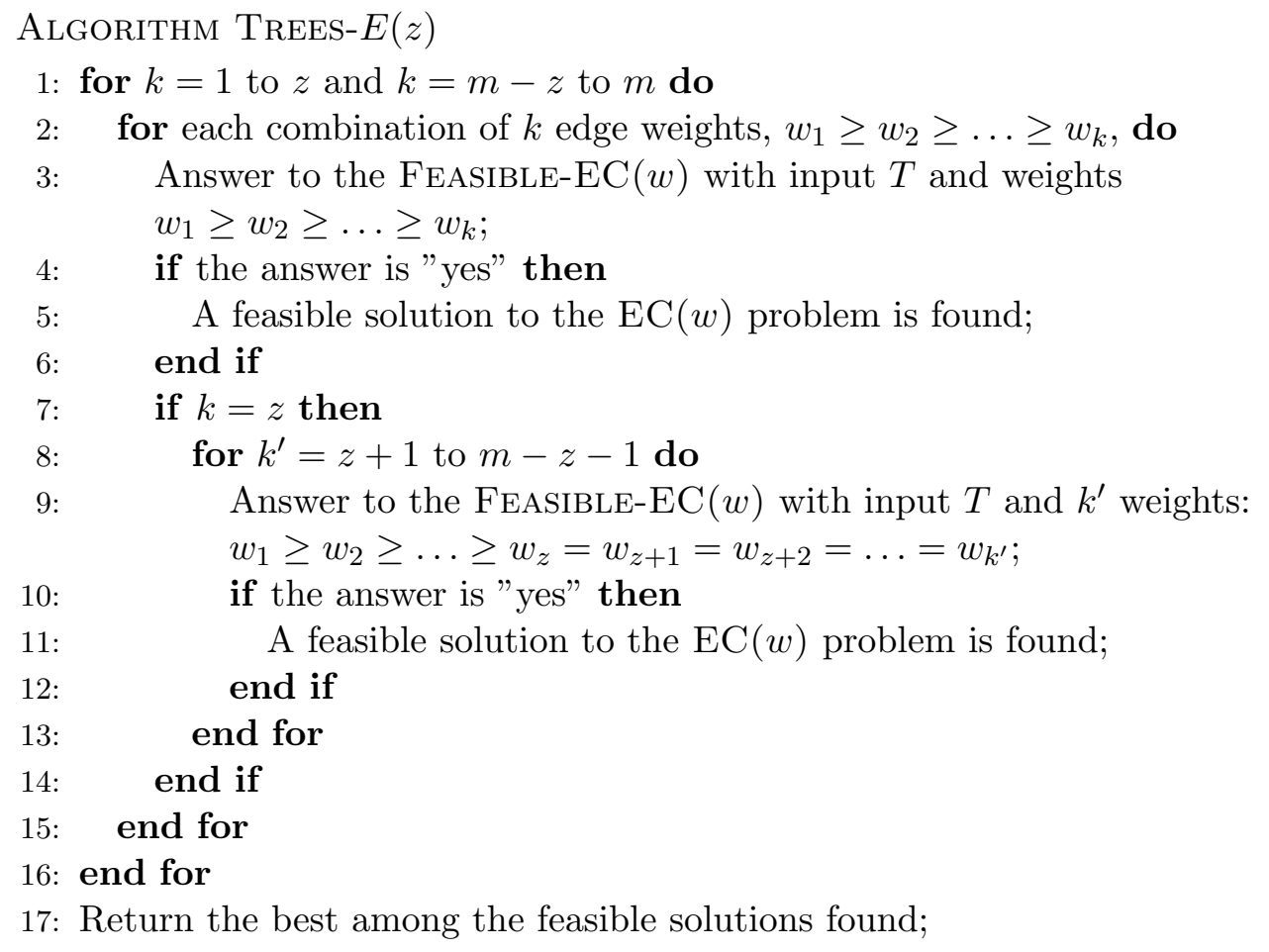


Theorem 17. For any $\rho \geq 1$, Algorithm Trees-E( $z)$ achieves a $\rho$ approximation ratio for the $\mathrm{EC}(w)$ problem on trees, in polynomial space and running time $O^{*}\left(g(\rho)^{m}\right)$, where $g(\rho)=\frac{(2 \rho-1)^{2}+1}{(2 \rho-1)^{2(2 \rho-1)^{2} /\left((2 \rho-1)^{2}+1\right)}}$.

Proof. If $k^{*} \leq z$ or $k^{*} \geq m-z$ then the algorithm in an iteration of Lines $3-6$ finds an optimal solution.

If $z<k^{*}<m-z$ then we consider the following two solutions found by the algorithm:

(i) In the iteration where $k=m-z$, for a combination $w_{1} \geq w_{2} \geq \ldots \geq w_{k}$ of weights, it holds that $w_{i}=w_{i}^{*}, 1 \leq i \leq k^{*}$. Hence, for this combination there is a feasible solution of weight at most $w_{1}^{*}+w_{2}^{*}+\ldots+w_{k^{*}}^{*}+\left(m-z-k^{*}\right) w_{k^{*}}^{*}=$ $O P T+\left(m-z-k^{*}\right) w_{k^{*}}^{*}$.

(ii) In the iteration where $k=z$ and $k^{\prime}=k^{*}$, for a combination $w_{1} \geq w_{2} \geq \ldots \geq w_{k}$ of weights, it holds that $w_{i}=w_{i}^{*}, 1 \leq i \leq z$. Hence, for this combination there is a feasible solution of weight at most $w_{1}^{*}+w_{2}^{*}+\ldots+w_{z}^{*}+\left(k^{*}-z\right) w_{z}^{*}=$ $O P T-\sum_{i=z+1}^{k^{*}} w_{i}^{*}+\left(k^{*}-z\right) w_{z}^{*}$.

Thus, it holds that

$$
\begin{aligned}
\frac{W}{O P T} & \leq \min \left\{\frac{O P T+\left(m-z-k^{*}\right) w_{k^{*}}^{*}}{O P T}, \frac{O P T-\sum_{i=z+1}^{k^{*}} w_{i}^{*}+\left(k^{*}-z\right) w_{z}^{*}}{O P T}\right\} \\
& \leq \min \left\{1+\frac{\left(m-z-k^{*}\right) w_{k^{*}}^{*}}{z w_{z}^{*}+\left(k^{*}-z\right) w_{k^{*}}^{*}}, 1+\frac{\left(k^{*}-z\right)\left(w_{z}^{*}-w_{k^{*}}^{*}\right)}{z w_{z}^{*}+\left(k^{*}-z\right) w_{k^{*}}^{*}}\right\} .
\end{aligned}
$$

As the first value is increasing with $w_{k^{*}}^{*}$ and the second one is decreasing, this quantity is maximized when $\left(k^{*}-z\right) w_{z}^{*}=(m-2 z) w_{k^{*}}^{*}$. Therefore, we have

$$
\frac{W}{O P T} \leq 1+\frac{\left(m-z-k^{*}\right) w_{k^{*}}^{*}}{\frac{z(m-2 z)}{k^{*}-z} w_{k^{*}}^{*}+\left(k^{*}-z\right) w_{k^{*}}^{*}}=\frac{k^{*}(m-2 z)}{z(m-2 z)+\left(k^{*}-z\right)^{2}},
$$

which is maximized for $k^{*}=\sqrt{z(m-z)}$. Hence,

$$
\frac{W}{O P T} \leq \frac{\sqrt{z(m-z)}(m-2 z)}{z(m-2 z)+(\sqrt{z(m-z)}-z)^{2}}=\frac{m-2 z}{2 \sqrt{z(m-z)}-2 z} .
$$

By setting $z=\lambda m$, where $0<\lambda \leq \frac{1}{2}$, we get

$$
\frac{W}{O P T} \leq \frac{m-2 \lambda m}{2 \sqrt{\lambda m(m-\lambda m)}-2 \lambda m}=\frac{1-2 \lambda}{2 \sqrt{\lambda(1-\lambda)}-2 \lambda}=\rho .
$$

Therefore, in order to achieve a $\rho$ approximation ratio we choose $\lambda=\frac{1}{(2 \rho-1)^{2}+1}$, that is $z=\frac{m}{(2 \rho-1)^{2}+1}$. 
The algorithm needs polynomial space, since Lines 3-14 are executed independently for each combination of weights. As the FEASIBLE-EC $(w)$ problem is polynomial for trees, the complexity of the algorithm is, within a polynomial factor, $O(T(m))$, where $T(m)$ is the number of combinations generated. For this number it holds that

$$
\begin{aligned}
T(m) & \leq \sum_{i=1}^{z}\left(\begin{array}{c}
m \\
i
\end{array}\right)+\sum_{i=m-z}^{m}\left(\begin{array}{c}
m \\
i
\end{array}\right)=2 \sum_{i=1}^{z}\left(\begin{array}{c}
m \\
i
\end{array}\right) \leq 2 z\left(\begin{array}{c}
m \\
z
\end{array}\right) \leq m\left(\begin{array}{c}
m \\
\lambda m
\end{array}\right) \\
& \leq m\left(\left(\frac{1}{\lambda}\right)^{\lambda}\left(\frac{1}{1-\lambda}\right)^{1-\lambda}\right)^{m}=m\left(\frac{(2 \rho-1)^{2}+1}{(2 \rho-1)^{2(2 \rho-1)^{2} /\left((2 \rho-1)^{2}+1\right)}}\right)^{m} \\
& =m \cdot g(\rho)^{m} .
\end{aligned}
$$

Hence, the complexity of Algorithm Trees- $E(z)$ becomes $O^{*}\left(g(\rho)^{m}\right)$, where $g(\rho)=$ $\frac{(2 \rho-1)^{2}+1}{(2 \rho-1)^{2(2 \rho-1)^{2} /\left((2 \rho-1)^{2}+1\right)}}$.

Note that for $z=\left\lfloor\frac{m}{2}\right\rfloor$ Algorithm Trees- $E(z)$ computes an optimal solution for the $\mathrm{EC}(w)$ problem on trees in $O^{*}\left(2^{m}\right)$ time and polynomial space.

In [5], an algorithm has been presented with running time and space $O^{*}\left(2^{n}\right)$, which, for any $k$, computes the number of all proper $k$-vertex-colorings of a graph, and moreover enumerates these colorings. This algorithm can be used to find an optimal solution for the $\mathrm{VC}(w)$ problem on a general graph, by running it for $1 \leq k \leq n$. Considering the line graph $L(G)$ of the input graph $G$ of the $\operatorname{EC}(w)$ problem, we derive that the $\mathrm{EC}(w)$ problem on general graphs can be optimally solved with running time and space $O^{*}\left(2^{m}\right)$.

Next proposition shows that if $\Delta=o(m)$ then the running time of AlGORITHM TreEs- $E(z)$ for computing an optimal solution is improved.

Proposition 7. If $\Delta=o(m)$, then Algorithm Trees-E $(z)$ requires subexponential running time $2^{o(m)}$ in order to compute an exact solution for trees.

Proof. By Proposition 2, the number $k^{*}$ of colors in any optimal solution to the $\mathrm{EC}(w)$ problem is at most $2 \Delta-1$. Thus, the number of combinations of weights needed to be generated by the algorithm becomes

$$
\begin{aligned}
T(m) & \leq\left(\begin{array}{c}
m \\
2 \Delta
\end{array}\right) \leq \frac{m^{m}}{(2 \Delta)^{2 \Delta}(m-2 \Delta)^{m-2 \Delta}} \\
& \leq 2^{m \log m-2 \Delta \log (2 \Delta)-(m-2 \Delta) \log (m-2 \Delta)} \\
& \leq 2^{m \log (1+2 \Delta /(m-2 \Delta))+2 \Delta \log (m / 2 \Delta-1)}
\end{aligned}
$$

Notice first that $2 \Delta /(m-2 \Delta)$ tends to 0 for $m \rightarrow \infty$, since $\Delta=o(m)$, and thus $m \log \left(1+\frac{2 \Delta}{(m-2 \Delta)}\right) \rightarrow 0$. Moreover, note that $2 \Delta \log \left(\frac{m}{2 \Delta}-1\right)=o(m)$, since $\frac{2 \Delta \log \left(\frac{m}{2 \Delta}-1\right)}{m}$ tends to 0 as $m$ increases. Combining the two observations 
above, we get that $T(m)=2^{o(m)}$ and, hence, the running time of Algorithm Trees- $E\left(\frac{m}{2}\right)$ is $O^{*}\left(2^{o(m)}\right)$.

Notice that Algorithm Trees- $E\left(\left\lfloor\frac{m}{2}\right\rfloor\right)$ and Algorithm Trees- $\Delta(2 \Delta-1)$ coincide and both return an optimal solution to the $\mathrm{EC}(w)$ problem on trees. Thus the last proposition holds for both algorithms.

\subsection{Polynomial Time Approximation Scheme}

In this section we combine the idea of iteratively splitting the input graph with Algorithm Trees in order to derive a PTAS for the $\mathrm{EC}(w)$ problem on trees.

In fact, our algorithm splits a tree $G=(V, E)$, into subgraphs $G\left[E_{1, j}\right]$ and $G\left[E_{j+1, m}\right]$ induced by the $j$ heaviest and the $n-j$ lightest edges of $G$, respectively (by convention, we consider $G\left[E_{1,0}\right]$ as an empty subgraph). The algorithm depends on a parameter $p$ such that all the edges of $G$ of weights $w_{1}^{*}, w_{2}^{*}, \ldots, w_{p-1}^{*}$ are in a subgraph $G\left[E_{1, j}\right]$. We obtain a solution for the whole graph by concatenating an optimal solution of at most $p-1$ colors for $G_{1, j}$, if there is one, and the solution obtained by Algorithm Trees for $G\left[E_{j+1, m}\right]$.

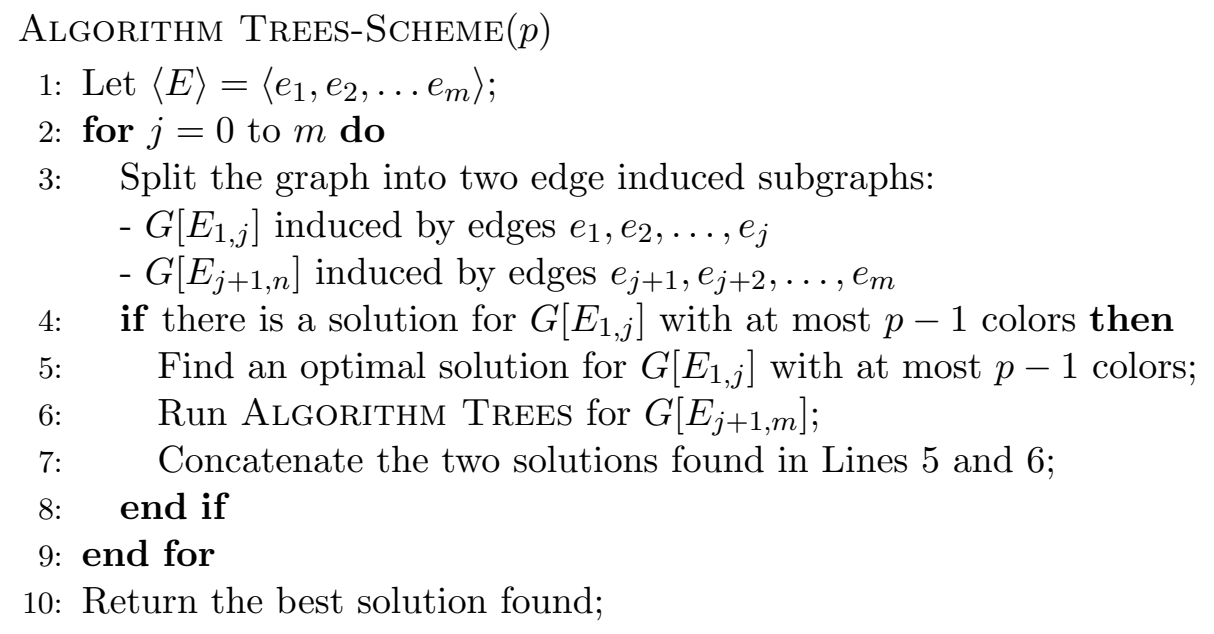

Theorem 18. Algorithm Trees-Scheme $(p)$ is a PTAS for the $\mathrm{EC}(w)$ problem on trees.

Proof. Consider the iteration $j, j \leq m$, of the algorithm where the weight of the heaviest edge in $G\left[E_{j+1, m}\right]$ equals to the weight of the $i$-th color of an optimal solution, i.e. $w\left(e_{j+1}\right)=w_{i}^{*}, 1 \leq i \leq p$.

The edges of $G\left[E_{1, j}\right]$ are a subset of those appeared in the $i-1$ heaviest colors of the optimal solution. Thus, an optimal solution for $G\left[E_{1, j}\right]$ is of weight

$$
O P T_{1, j} \leq w_{1}^{*}+w_{2}^{*}+\ldots+w_{i-1}^{*} .
$$


The edges of $G\left[E_{j+1, m}\right]$ are a superset of those appeared in the $k^{*}-(i-1)$ lightest colors of the optimal solution. The extra edges of $G\left[E_{j+1, m}\right]$ are of weight at most $w_{i}^{*}$ and appear in an optimal solution into at most $i-1$ colors. Thus, an optimal solution for $G\left[E_{j+1, m}\right]$ is of weight

$$
O P T_{j+1, m} \leq w_{i}^{*}+w_{i+1}^{*}+\ldots+w_{k^{*}}^{*}+(i-1) \cdot w_{i}^{*}=i \cdot w_{i}^{*}+w_{i+1}^{*}+\ldots+w_{k^{*}}^{*} .
$$

By Lemma 4, Algorithm Trees returns a solution for $G\left[E_{j+1, m}\right]$ of weight

$$
\begin{aligned}
W_{j+1, m} & \leq O P T_{j+1, m}+w_{i}^{*}-w_{\Delta}^{*} \\
& \leq i \cdot w_{i}^{*}+w_{i+1}^{*}+\ldots+w_{k^{*}}^{*}+w_{i}^{*} \\
& \leq(i+1) \cdot w_{i}^{*}+w_{i+1}^{*}+\ldots+w_{k^{*}}^{*}
\end{aligned}
$$

Therefore, the solution found in this iteration $j$ for the whole graph $G$ is of weight

$$
W_{i}=O P T_{1, j}+W_{j+1, m} \leq w_{1}^{*}+w_{2}^{*}+\ldots+w_{i-1}^{*}+(i+1) \cdot w_{i}^{*}+w_{i+1}^{*}+\ldots+w_{k^{*}}^{*} .
$$

In all the iterations of the algorithm we obtain $p$ such inequalities for $W$. By multiplying the $i$-th, $1 \leq i \leq p$, inequality by $\frac{1}{i \cdot\left(H_{p}+1\right)}$ and adding up all of them, we have $\left(\sum_{i=1}^{p} \frac{1}{i \cdot\left(H_{p}+1\right)}\right) \cdot W \leq O P T$, that is $\frac{W}{O P T} \leq \frac{H_{p}+1}{H_{p}}=1+\frac{1}{H_{p}}$.

Algorithm Trees-Scheme $(p)$ iterates $|E|$ times. In each iteration: (i) an optimal solution, if any, with at most $p-1$ colors for $G\left[E_{1, j}\right]$ is found using Theorem 2(i), in $O\left(|E|^{p-1} \cdot|E| \cdot \Delta^{3.5}\right)$ time, and (ii) Algorithm Trees of complexity $O(|V|$. $\Delta \cdot \log \Delta)$ is called for $G\left[E_{j+1, m}\right]$. Choosing $p$ such that $\epsilon=\frac{1}{H_{p}}$, we get $p=O\left(2^{\frac{1}{\epsilon}}\right)$. Consequently, we have a PTAS for the $\mathrm{EC}(w)$ problem on trees, that is an approximation ratio of $1+\frac{1}{H_{p}}=1+\epsilon$ within time $O\left(|E|\left(|V| \cdot \Delta \cdot \log \Delta+|E|^{p} \cdot \Delta^{3.5}\right)\right)$ which is exponential to $\frac{1}{\epsilon}$. 


\section{Chapter 6}

\section{Bounded Max-Edge-Coloring}

In this chapter we deal with the complexity and approximability of the $\operatorname{EC}(w, b)$ problem. First, we present an approximation algorithm for general and bipartite graphs. Then, we prove that the problem is NP-complete for trees and we give a 2-approximation algorithm for this case. Note that this is the first complexity result for any max-coloring problem on trees.

\subsection{General and bipartite graphs}

Our approximation algorithm for general and bipartite graphs is based on tight bounds on the number of colors in a solution to the $\mathrm{EC}(w, b)$ problem. In fact, our bounds apply to any nice solution $\langle\mathcal{C}\rangle=\left\langle C_{1}, C_{2}, \ldots, C_{k}\right\rangle$ to the $\mathrm{EC}(w, b)$ problem. We call such a solution nice if each color $C_{i}, 1 \leq i \leq k$, is of cardinality $\left|C_{i}\right|=b$ or $C_{i}$ is maximal in the subgraph induced by the edges $\bigcup_{j=i}^{k} C_{j}$. It is easy to see that any solution to the $\mathrm{EC}(w, b)$ problem can be transformed into a nice one of the same total weight.

Proposition 8. For the number, $k$, of colors in any nice solution to the $\mathrm{EC}(w, b)$ problem it holds that:

$$
\max \left\{\Delta,\left\lceil\frac{|E|}{b}\right\rceil\right\} \leq k \leq\left\{\begin{array}{l}
\left\lceil\frac{|E|}{b}\right\rceil-\left\lceil\frac{\Delta^{2}}{2 b}\right\rceil+(2 \Delta-1), \text { for general graphs } \\
\left\lceil\frac{|E|}{b}\right\rceil-\left\lceil\frac{\Delta^{2}}{b}\right\rceil+(2 \Delta-1), \text { for bipartite graphs }
\end{array}\right.
$$

Proof. The lower bounds follow trivially. For the upper bounds, let $\langle\mathcal{C}\rangle=\left\langle C_{1}, C_{2}\right.$, $\left.\ldots, C_{k}\right\rangle$ be a nice solution, $e=(u, v)$ be an edge in the last color $C_{k}$, and $E_{u}$ and $E_{v}$ be the sets of edges adjacent to vertices $u$ and $v$, respectively. By the niceness of the solution $\mathcal{C}$ it follows that edge $e$ does not appear in any color $C_{i}$, $1 \leq i \leq k-1$, because $\left|C_{i}\right|=b$ or $C_{i}$ contains at least one edge in $E_{u}$ or $E_{v}$. Let $W, X, Y \subseteq\left\{C_{1}, C_{2}, \ldots, C_{k-1}\right\}$ such that $W=\left\{C_{i}:\left|C_{i}\right|=b\right\}, X=\left\{C_{i}:\left|C_{i}\right|<b\right.$ and $C_{i}$ contains an edge $\left.e \in E_{u}\right\}$ and $Y=\left\{C_{i}:\left|C_{i}\right|<b\right.$ and $C_{i}$ contains an edge $\left.e \in E_{v}\right\}$. Let $E_{1}$ be the set of edges in the colors in $W$ and $E_{2}=E \backslash E_{1}$ be the set of edges in the colors in $X \cup Y \cup\left\{C_{k}\right\}$. Then, $k=\frac{\left|E_{1}\right|}{b}+x+y+1$, where $x=|X|$ and $y=|Y|$. 
Assume, w.l.o.g., that $X_{1} Y_{1} X_{2} Y_{2} \ldots X_{l} Y_{l}$ is the order of colors in the nice solution $\langle\mathcal{C}\rangle$, where $X_{i} \subseteq X, Y_{i} \subseteq Y, 1 \leq i \leq l$, and $X_{1}$ is possibly empty. Let $x_{i}=\left|X_{i}\right|$ and $y_{i}=\left|Y_{i}\right|, 1 \leq i \leq l$.

For general graphs, consider a color $C \in X_{i}$ and let $S_{i}=\bigcup_{j=i}^{l} Y_{j}$ and $s_{i}=\sum_{j=i}^{l} y_{j}$. The edge $(u, z) \in C \cap E_{u}$ prevents at most one edge $(v, z) \in S_{i} \cap E_{v}$ from being into $C$. Moreover, each other edge $(p, q) \in C$ prevents at most two edges $(v, p),(v, q) \in$ $S_{i} \cap E_{v}$ from being into $C$. As the colors in $S_{i}$ contain exactly $s_{i}$ edges from $E_{v}$ and all of them are prevented from being into color $C$, it follows that $|C| \geq\left\lceil\frac{s_{i}-1}{2}\right\rceil+1$. Therefore, there exist at least $x_{i} \cdot\left\lceil\frac{s_{i}-1}{2}\right\rceil+x_{i}$ edges in $X_{i}$. In a similar way, by considering a color $C \in Y_{i}$ there exist at least $y_{i} \cdot\left\lceil\frac{t_{i}-1}{2}\right\rceil+y_{i}$ edges in $Y_{i}$, where $t_{i}=\sum_{j=i+1}^{l} x_{j}$. Summing up these bounds, and taking into account that $y_{l}-1 \geq 0$ (since $Y_{l}$ is not empty), it follows that

$$
\begin{aligned}
\left|E_{2}\right| & \geq \sum_{i=1}^{l}\left(x_{i} \cdot\left\lceil\frac{s_{i}-1}{2}\right\rceil+x_{i}\right)+\sum_{i=1}^{l}\left(y_{i} \cdot\left\lceil\frac{t_{i}-1}{2}\right\rceil+y_{i}\right) \\
& =\frac{x(y-1)-\left(y_{1}+y_{2}+\ldots+y_{l-1}\right)}{2}+x+y+1 \\
& \geq \frac{x(y-1)-\left(y_{1}+y_{2}+\ldots+y_{l-1}+y_{l}-1\right)}{2}+x+y+1 \\
& =\frac{(x-1)(y-1)}{2}+x+y+1 \geq \frac{(x+1)(y+1)}{2}+1 \geq\left\lceil\frac{(x+1)(y+1)}{2}\right\rceil .
\end{aligned}
$$

Therefore, $k=\frac{\left|E \backslash E_{2}\right|}{b}+x+y+1 \leq\left\lceil\frac{|E|}{b}\right\rceil-\left\lceil\frac{(x+1)(y+1)}{2 b}\right\rceil+x+y+1$ If $\Delta \leq 2 b$ then this quantity is maximized when $x=y=\Delta-1$ and hence $k \leq\left\lceil\frac{|E|}{b}\right\rceil-\left\lceil\frac{\Delta^{2}}{2 b}\right\rceil+(2 \Delta-1)$. If $\Delta>2 b$ then the above quantity is maximized when $x=\Delta-1$ and $y=0$ and hence $k \leq\left\lceil\frac{|E|}{b}\right\rceil-\left\lceil\frac{\Delta}{2 b}\right\rceil+\Delta \leq\left\lceil\frac{|E|}{b}\right\rceil-\left\lceil\frac{\Delta^{2}}{2 b}\right\rceil+(2 \Delta-1)$.

For bipartite graphs, the proof is similar. The structure of a bipartite graph allows a tighter bound on the number of edges in the colors in $X_{i}$ and $Y_{i}$. Consider, again, a color $C \in X_{i}$. For the edge $(u, z) \in C \cap E_{u}$, there is no edge $(v, z) \in S_{i} \cap E_{v}$, while each other edge $(p, q) \in C$ prevents at most one edge $(v, p)$ or $(v, q)$ in $S_{i} \cap E_{v}$ from being into $C$. Thus, $|C| \geq s_{i}+1$ and, hence, there exist at least $x_{i}\left(s_{i}+1\right)$ edges in $X_{i}$. Similarly there exist at least $y_{i}\left(t_{i}+1\right)$ edges in $Y_{i}$. The rest of the proof is along the same lines, but using these bounds.

Our algorithm for general and bipartite graphs adapts the greedy 2-approximation algorithm presented in [46] for the $\mathrm{EC}(w)$ problem to the $\mathrm{EC}(w, b)$ problem.

\section{Algorithm Greedy}

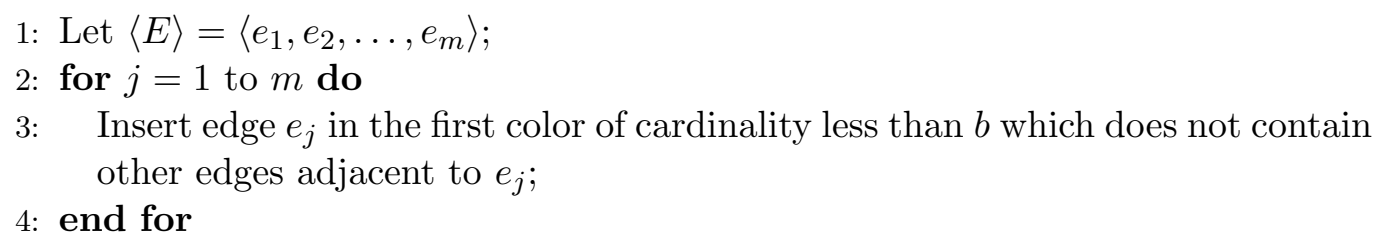


The solution derived by Algorithm Greedy is a nice one, since it is constructed in a first-fit manner. The analysis of this algorithm given in the next lemma is based on Proposition 8.

Lemma 5. Algorithm GREedy achieves approximation ratios of $3-\frac{2}{\sqrt{2 b}}$ on general graphs, and $3-\frac{2}{\sqrt{b}}$ on bipartite graphs, for the $\mathrm{EC}(w, b)$ problem.

Proof. Let $\langle\mathcal{C}\rangle=\left\langle C_{1}, C_{2}, \ldots, C_{k}\right\rangle$, be a solution derived by Algorithm Greedy. Consider the color $C_{i}$ and let $e_{j}$ be the first edge inserted in $C_{i}$, i.e. $w_{i}=w\left(e_{j}\right)$. Let $E_{i}=\left\{e_{1}, e_{2}, \ldots, e_{j}\right\}, G_{i}$ be the subgraph of $G$ induced by the edges in $E_{i}$, and $\Delta_{i}$ be the maximum degree of $G_{i}$.

As the solution $\langle\mathcal{C}\rangle$ is a nice one and an optimal solution can be also considered to be nice, by Proposition 8, for general graphs, it follows that (i) $i \leq\left\lceil\frac{\left|E_{i}\right|}{b}\right\rceil-$ $\left\lceil\frac{\Delta_{i}^{2}}{2 b}\right\rceil+\left(2 \Delta_{i}-1\right)$, and (ii) in an optimal solution the edges of $G_{i}$ appear in at least $i^{*} \geq \max \left\{\Delta_{i},\left\lceil\frac{\left|E_{i}\right|}{b}\right\rceil\right\}$ colors, each one of weight at least $w_{i}$. Therefore, $\frac{i}{i^{*}} \leq$ $\frac{\left\lceil\frac{\left|E_{i}\right|}{b}\right\rceil-\left\lceil\frac{\Delta_{i}^{2}}{2 b}\right\rceil+\left(2 \Delta_{i}-1\right)}{\max \left\{\Delta_{i},\left\lceil\frac{\left|E_{i}\right|}{b}\right\rceil\right\}}$. By distinguish between $\Delta_{i} \geq\left\lceil\frac{\left|E_{i}\right|}{b}\right\rceil$ and $\Delta_{i}<\left\lceil\frac{\left|E_{i}\right|}{b}\right\rceil$ it follows that in either case $\frac{i}{i^{*}} \leq 3-\frac{\Delta_{i}^{2}+2 b}{2 b \Delta_{i}}$. This bound is maximized when $\Delta_{i}=\sqrt{2 b}$, that is $\frac{i}{i^{*}} \leq 3-\frac{2}{\sqrt{2 b}}$. Thus, $\left.w_{i} \leq w_{i^{*}}^{*} \leq w_{\left\lceil i /\left(3-\frac{2}{\sqrt{2 b}}\right)\right.}^{*}\right\rceil^{*}$ Summing up these inequalities for all $i$ 's, $1 \leq i \leq k$, we obtain the $\left(3-\frac{2}{\sqrt{2 b}}\right)$ ratio for general graphs.

A similar analysis yields the $\left(3-\frac{2}{\sqrt{b}}\right)$ ratio for bipartite graphs.

We present here an example for which the algorithm performs a ratio of $3-\frac{2}{\sqrt{2 b}}$ for general graphs. Consider the general graph shown in Figure 6.1(a), where $1>>\epsilon$, and $b=7$. The weight of the optimal solution shown in Figure 6.1(b) is $3+3 \epsilon$. The weight of the solution obtained by ALGORITHM GreEDY, shown in Figure 6.1(c), is $7-\epsilon$. Thus, the ratio for this instance is $\frac{7-\epsilon}{3+3 \epsilon} \simeq \frac{7}{3} \simeq 3-\frac{2}{\sqrt{14}}$.

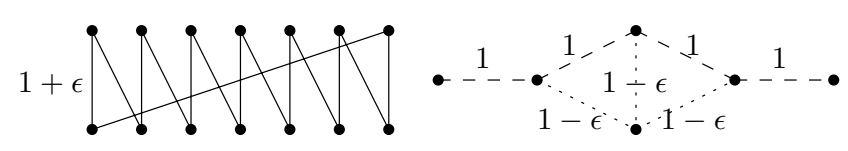

(a)

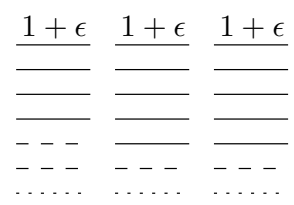

(b)

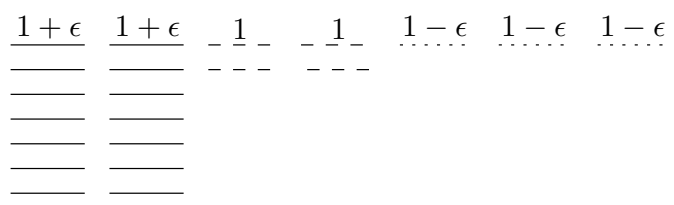

(c)

Figure 6.1: (a) An instance of the $\mathrm{EC}(w, b)$ problem on general graphs, $1>>\epsilon$, $b=7$. (b) An optimal solution. (c) The solution obtained by Algorithm Greedy. 
Consider, now, the bipartite graph shown in Figure 6.2(a), where $1>>\epsilon$, and $b=9$. The optimal solution is of weight $3+3 \epsilon$ (Figure $6.2(\mathrm{a})$ ), while the weight of the solution obtained by Algorithm Greedy is $7-\epsilon$ (Figure 6.2(c)). Hence, the ratio for this instance is $\frac{7-\epsilon}{3+3 \epsilon} \simeq \frac{7}{3}=3-\frac{2}{\sqrt{9}}=3-\frac{2}{\sqrt{b}}$.

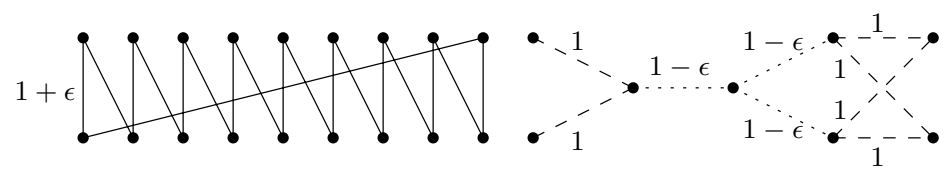

(a)

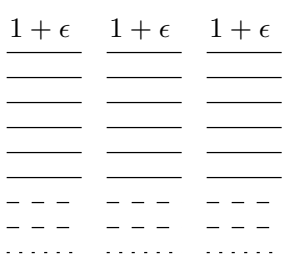

(b)

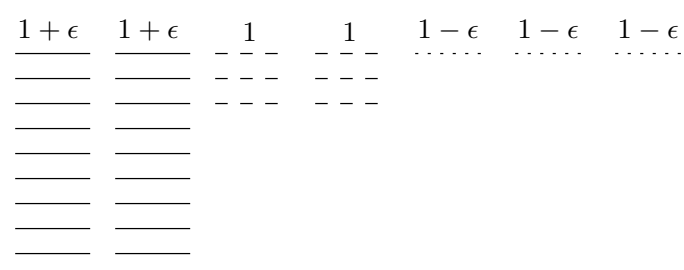

(c)

Figure 6.2: (a) An instance of the $\mathrm{EC}(w, b)$ problem on bipartite graphs, $1>>\epsilon$, $b=9$. (b) An optimal solution. (c) The solution obtained by Algorithm Greedy.

Combining Lemma 5 and Theorems 3, 4 and 5, it follows that

Theorem 19. The $\mathrm{EC}(w, b)$ problem can be approximated with a ratio of $\min \{3-$ $\left.2 / \sqrt{2 b}, H_{b},\lceil b / 2\rceil\right\}$ for general graphs and $\min \left\{e, 3-2 / \sqrt{b}, H_{b},\lceil b / 2\rceil\right\}$ for bipartite graphs.

Note that, the $H_{b}$ ratio outperforms the other only for $b=3$ or 5 , for general graphs, and $b=3$, for bipartite graphs, and, hence, $b$ can be considered as fixed. These ratios are shown in Table 6.1, for several values of $b$.

\begin{tabular}{|c||ll|ll|}
\hline \multicolumn{1}{|c||}{$b$} & \multicolumn{2}{c|}{ General graphs } & \multicolumn{2}{c|}{ Bipartite graphs } \\
\hline \hline 3 & 1.833 & $H_{b}$ & 1.833 & $H_{b}$ \\
4 & 2.000 & $\lceil b / 2\rceil$ & 2.000 & $3-2 / \sqrt{b}$ or $\lceil b / 2\rceil$ \\
5 & 2.283 & $H_{b}$ & 2.106 & $3-2 / \sqrt{b}$ \\
6 & 2.423 & $3-2 / \sqrt{2 b}$ & 2.184 & $3-2 / \sqrt{b}$ \\
$\ldots$ & $\ldots$ & $3-2 / \sqrt{2 b}$ & $\ldots$ & $3-2 / \sqrt{b}$ \\
50 & 2.800 & $3-2 / \sqrt{2 b}$ & 2.717 & $3-2 / \sqrt{b}$ \\
51 & 2.802 & $3-2 / \sqrt{2 b}$ & 2.718 & $e$ \\
$\ldots$ & $\ldots$ & $3-2 / \sqrt{2 b}$ & $\ldots$ & $e$ \\
\hline
\end{tabular}

Table 6.1: Approximation ratios for the $\mathrm{EC}(w, b)$ problem. 


\subsection{NP-completeness for trees}

To prove that the $\operatorname{EC}(w, b)$ problem is NP-complete on trees, we will present a reduction from the bounded list vertex-coloring problem, $\mathrm{VC}\left(\phi, b_{i}\right)$. By Theorem 1(iii), $\mathrm{VC}\left(\phi, b_{i}\right)$ problem is NP-complete even for chains, $|\phi(u)| \leq 2$, for all $u \in V$, and $b_{i} \leq 5,1 \leq i \leq k$.

We prove first that the bounded list edge-coloring, $\mathrm{EC}(\phi, b)$, problem is NPcomplete even if the graph $G=(V, E)$ is a set of chains, $|\phi(e)|=2$, for all $e \in E$, and $b=5$. We denote this problem as $\mathrm{EC}($ chains, $|\phi(e)|=2, b=5)$.

Proposition 9. The EC(chains, $|\phi(e)|=2, b=5)$ problem is NP-complete.

Proof. By Theorem 1(iii), the $\mathrm{VC}\left(\right.$ chains, $\left.|\phi(v)| \leq 2, b_{i} \leq 5\right)$ problem is NPcomplete. Given that the line-graph of a chain is also a chain, it follows that the $\mathrm{EC}$ (chains, $\left.|\phi(e)| \leq 2, b_{i} \leq 5\right)$ problem is also NP-complete. The latter problem can be easily reduced to the $\mathrm{EC}\left(\right.$ chains, $|\phi(e)| \leq 2, b=5$ ) problem, where $b_{i}=b=5$ for all colors: for every color $C_{i}$ with $b_{i}<5$, add $5-b_{i}$ independent edges with just $C_{i}$ in their lists. This last problem reduces to the $\mathrm{EC}($ chains, $|\phi(e)|=2, b=5)$ problem, where $|\phi(e)|=2$ for all edges. This can be done by transforming an instance of EC(chains, $|\phi(v)| \leq 2, b=5$ ) as following: (i) add two new colors $C_{k+1}$ and $C_{k+2}$, both with cardinality bound $b=5$, (ii) add color $C_{k+1}$ to the list of every edge $e$ with $|\phi(e)|=1$, (iii) add ten independent edges and put in their lists both colors $C_{k+1}$ and $C_{k+2}$.

Theorem 20. The $\mathrm{EC}(w, b)$ problem on trees is NP-complete.

Proof. Our reduction is from $\mathrm{EC}($ chains, $|\phi(e)|=2, b=5)$ problem. We construct an instance of the $\operatorname{EC}(w, b)$ problem on a forest $G^{\prime}=\left(V^{\prime}, E^{\prime}\right)$ as follows.

We replace every edge $e=(u, v) \in E$ with a chain of three edges: $e_{1}=\left(u, u^{\prime}\right)$, $e_{2}=\left(u^{\prime}, v^{\prime}\right)$ and $e_{3}=\left(v^{\prime}, v\right)$, where $w\left(e_{1}\right)=w\left(e_{2}\right)=w\left(e_{3}\right)=1$. Moreover, we create $k-|\phi(e)|=k-2$ stars of $k-1$ edges each. We add edges $\left(u^{\prime}, s_{t}\right), 1 \leq t \leq k-2$, between $u^{\prime}$ and the central vertex $s_{t}$ of each of these $k-2$ stars; thus every star has now exactly $k$ edges. Let $\phi(e)=\left\{C_{i}, C_{j}\right\}$. The $k-2$ edges $\left(u^{\prime}, s_{t}\right)$ take different

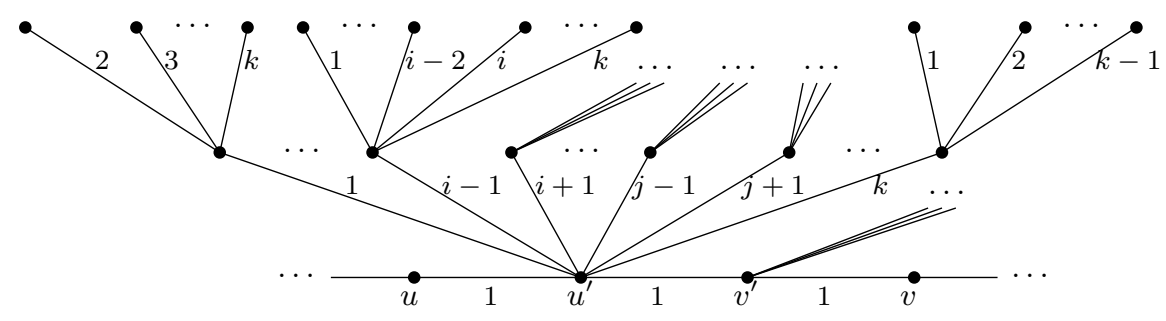

Figure 6.3: The gadget for an edge $e=(u, v)$ with $\phi(e)=\left\{C_{i}, C_{j}\right\}$.

weights in $\{1,2, \ldots, k\} \backslash\{i, j\}$. Let $q$ be the weight taken by an edge $\left(u^{\prime}, s_{t}\right)$. The remaining $k-1$ edges of the star $t$ take different weights in $\{1,2, \ldots, k\} \backslash\{q\}$. In the same way, we add $k-2$ stars connected to $v^{\prime}$. In Figure 6.3 , is shown the $u^{\prime \prime}$ s 
part of this edge-gadget for $e=(u, v)$. For every edge $e$ of $G$, we add $2(k-2)$ stars and $2(k-2) k+2$ edges.

To complete our construction we define $f_{i}$ to be the frequency of color $C_{i}$ in the lists of all edges and $F=\max \left\{f_{i} \mid 1 \leq i \leq k\right\}$. For every color $C_{i}$ we add $F-f_{i}$ disconnected copies of the color-gadget shown in Figure 6.4. Such a gadget consists of an edge $e=(x, y)$ and $k-1$ stars with $k-1$ edges each. There are also edges between one of the endpoints of $e$, say $y$, and the central vertices of all stars; thus every star has now exactly $k$ edges. The edge $e$ takes weight $i$ and the edges in the stars of such a color-gadget take weights similarly with those in the stars of an edgegadget. For a color $C_{i}$ we add $\left(F-f_{i}\right)(k-1)$ stars and $\left(F-f_{i}\right)(k-1) k+\left(F-f_{i}\right)$ edges.

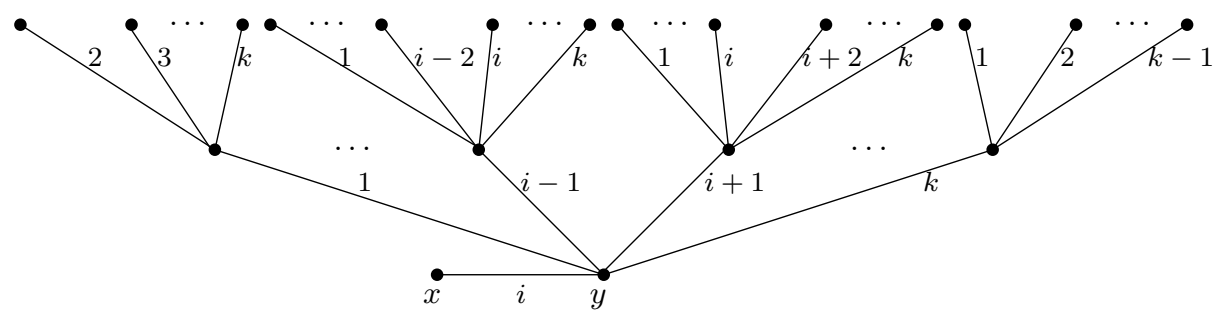

Figure 6.4: A gadget for the color $C_{i}$.

The number of stars in the forest $G^{\prime}$ we have constructed is $2|E|(k-2)+\sum_{i=1}^{k}(F-$ $\left.f_{i}\right)(k-1)=k(k-1) F-2|E|$, since $\sum_{i=1}^{k} f_{i}=2|E|$. By setting $b^{\prime}=k(k-1) F-2|E|+$ $5+F$, we prove that: "There is a $k$-coloring for $\operatorname{EC}(\phi, b)$ (chains, $|\phi(e)|=2, b=5$ ), if and only if, $G^{\prime}$ has a bounded max-edge-coloring of total weight $\sum_{i=1}^{k} i$ such that every color is used at most $b^{\prime}$ times".

Consider, first, a solution $\mathcal{C}$ to the $\operatorname{EC}(\phi, b)$ problem. We construct a solution $\mathcal{C}^{\prime}$ for the $\operatorname{EC}(w, b)$ problem as following. Let $e=(u, v) \in E$ be an edge with $\phi(e)=\left\{C_{i}, C_{j}\right\}$, which, w.l.o.g., appears in the color $C_{i}$ of $\mathcal{C}$. Put the edges $e_{1}$ and $e_{3}$ of the edge-gadget for $e$ in color $C_{i}^{\prime}$, while the edge $e_{2}$ in color $C_{j}^{\prime}$. After doing this for all edges in $E$, each color $C_{i}^{\prime}$ contains at most $2 \cdot 5+1 \cdot\left(f_{i}-5\right)=f_{i}+5$ edges. Next, put the edges with weight $i, 1 \leq i \leq k$, from the $k(k-1) F-2|E|$ stars into $C_{i}^{\prime}$. Each color $C_{i}^{\prime}$ in $\mathcal{C}^{\prime}$ constructed so far contains at most $k(k-1) F-2|E|+f_{i}+5=b^{\prime}-\left(F-f_{i}\right)$ edges and, by the construction of $G^{\prime}, \mathcal{C}^{\prime}$ is a proper coloring. In the $F-f_{i}$ colorgadgets for $C_{i}$ there are $F-f_{i}$ remaining $(x, y)$ edges of weight $i$, which can still be inserted into color $C_{i}^{\prime}$. Thus, we get a solution for the $\mathrm{EC}(w, b)$ problem of $k$ colors, each one of at most $b^{\prime}$ edges, and total weight $\sum_{i=1}^{k} i$.

Conversely, consider a solution $\mathcal{C}^{\prime}$ to the $\mathrm{EC}(w, b)$ problem. $\mathcal{C}^{\prime}$ consists of exactly $k$ colors of weights $1,2, \ldots, k$, since each star in $G^{\prime}$ has $k$ edges and each edge has a different weight in the range $\{1,2, \ldots, k\}$. Thus, all edges of the same weight, say $i$, should belong in the same color $C_{i}^{\prime}$ of $\mathcal{C}^{\prime}$. Therefore, $C_{i}^{\prime}$ contains one edge from each one of the $k(k-1) F-2|E|$ stars as well as the $F-f_{i}$ remaining $(x, y)$ edges of the color-gadgets having weight $i$. Consider, now, the edges of $G^{\prime}$ corresponding 
to the edges $e_{1}, e_{2}$ and $e_{3}$ of the edge-gadget for an edge $e$ with $\phi(e)=\left\{C_{i}, C_{j}\right\}$. By the construction of $G^{\prime}$ and the choice of edge weights, the edges $e_{1}, e_{2}$ and $e_{3}$ should appear into colors $C_{i}^{\prime}$ and $C_{j}^{\prime}$. Thus, edges $e_{1}$ and $e_{3}$ should appear, w.l.o.g., into color $C_{i}^{\prime}$, while $e_{2}$ into color $C_{j}^{\prime}$. Therefore, the edge $e \in E$ can be colored by color $C_{i} \in \phi(e)$. Finally, a color $C_{i}^{\prime}$ contains at most 5 edges of type $e_{1}$ (or $e_{3}$ ), corresponding to at most 5 edges of $E$; otherwise $\left|C_{i}^{\prime}\right| \geq k(k-1) F-2|E|+(F-$ $\left.f_{i}\right)+\left(2 \cdot 6+1 \cdot\left(f_{i}-6\right)\right)>b^{\prime}$, a contradiction.

To complete our proof for the $\mathrm{EC}(w, b)$ problem on trees, let $p$ be the number of trees in $G^{\prime}$. We add a set of $p-1$ edges of weight $\epsilon<1$ to transform the forest $G^{\prime}$ into a single tree $T$. This can be done as every tree of $G^{\prime}$ has at least two vertices. By keeping the same bound $b^{\prime}$, it is easy to see that there is a solution for the $\operatorname{EC}(w, b)$ problem on $G^{\prime}$ of weight $\sum_{i=1}^{k} i$, if and only if, there is a solution for the $\operatorname{EC}(w, b)$ problem on $T$ whose weight is equal to $\sum_{i=1}^{k} i+\left\lceil\frac{p-1}{b^{\prime}}\right\rceil \epsilon$.

\subsection{A 2-approximation algorithm for trees}

In Section 5.2 a 2-approximation algorithm for the $\mathrm{EC}(w)$ problem on trees has been presented, which is also exploited to derive a ratio of $3 / 2$ for that problem. This algorithm yields a solution of $\Delta$ colors, $\mathcal{M}=\left\{M_{1}, M_{2}, \ldots, M_{\Delta}\right\}$. Starting from this solution we obtain a solution to the $\operatorname{EC}(w, b)$ problem by finding the ordered $b$-partition of each color in $\mathcal{M}$. For the sake of completeness we give below the whole algorithm.

\section{Algorithm Convert}

1: Let $T_{r}$ be the tree rooted in an arbitrary vertex $r$;

2: for each vertex $v$ in pre-order traversal of $T_{r}$ do

3: $\quad$ Let $\left\langle E_{v}\right\rangle=\left\langle e_{1}, e_{2}, \ldots, e_{d(v)}\right\rangle$ be the edges adjacent to $v$, and $(v, p)$ be the edge from $v, v \neq \equiv$, to its parent;

4: $\quad$ Using ordering $\left\langle E_{v}\right\rangle$, insert each edge in $E_{v}$, but $(v, p)$, into the first color which does not contain an edge in $E_{v}$;

5: end for

6: Let $\mathcal{M}=\left\{M_{1}, M_{2}, \ldots, M_{\Delta}\right\}$ be the colors constructed;

7: for $i=1$ to $\Delta$ do

8: $\quad$ Let $\mathcal{P}_{M_{i}}=\left\{M_{1}^{i}, M_{2}^{i}, \ldots, M_{k_{i}}^{i}\right\}$ be the ordered $b$-partition of $\left\langle M_{i}\right\rangle$;

9: end for

10: Return a solution $\langle\mathcal{C}\rangle=\left\langle C_{1}, C_{2}, \ldots, C_{k}\right\rangle, \mathcal{C}=\bigcup_{i=1}^{\Delta} \mathcal{P}_{M_{i}}$;

Theorem 21. Algorithm Convert is a 2-approximation one for the $\mathrm{EC}(w, b)$ problem on trees.

Proof. Consider the color $C_{j}$ in the solution $\langle\mathcal{C}\rangle$ and let $e$ be the heaviest edge in $C_{j}$, i.e., $w(e)=w_{j}$. Let $X \subseteq \mathcal{C}_{j}=\left\{C_{1}, C_{2}, \ldots, C_{j-1}\right\}$ such that each color $C_{p} \in X$ has (i) $\left|C_{p}\right|=b$, and (ii) all edges of weight at least $w(e)$. Let also $Y=\mathcal{C}_{j} \backslash X$, 
$|X|=x$ and $|Y|=y$. Clearly, $x+y=j-1$. Let $j^{*}$ be the number of colors in an optimal solution of weight at least $w(e)$, that is $w_{j} \leq w_{j^{*}}^{*}$.

There are at least $x \cdot b+y+1$ edges of weight at least $w(e)$. These edges in an optimal solution appear in at least $\left[\frac{x \cdot b+y+1}{b}\right] \geq x+1$ colors, that is, $j^{*} \geq x+1$.

We show, next, that all colors in $Y \cup\left\{C_{j}\right\}$ come from $y+1$ different colors in $\mathcal{M}$. Assume that two of these colors, $C_{q}$ and $C_{r}$, come from the ordered $b$-partition of the same color $M_{t} \in \mathcal{M}$. Assume, w.l.o.g., that $w_{q} \geq w_{r}$, and let $f$ be the heaviest edge in $C_{r}$. Note that $C_{r}$ may coincide with $C_{j}$, while $C_{q}$ cannot. As $C_{q} \in Y$, it follows that $\left|C_{q}\right|=b$ and there is an edge $f^{\prime} \in C_{q}$ with $w\left(f^{\prime}\right)<w(e) \leq w(f)$, a contradiction to the definition of the ordered $b$-partition of $M_{t}$. Therefore, $C_{j}$ comes from a color $M_{i} \in \mathcal{M}, i \geq y+1$, that is $e \in M_{i}$. By the construction of the coloring $\mathcal{M}$, there are at least $i-1$ edges, adjacent to each other, of weight at least $w(e)$ (i.e., $i-2$ of them adjacent to $e$ and $e$ itself). These $i-1$ edges appear in different colors in an optimal solution, that is, $j^{*} \geq y$.

Combining the two lower bounds for $j^{*}$ and taking into account that $x+y=j-1$ we get $j^{*} \geq\left\lceil\frac{j}{2}\right\rceil$. Therefore, $w_{j}=w_{j^{*}}^{*} \leq w_{\left\lceil\frac{j}{2}\right\rceil}^{*}$ and summing up the weights $w_{j}$ of all colors in $\mathcal{C}$ we get $W=\sum_{j=1}^{k} w_{j} \leq 2 \sum_{j=1}^{\lceil k / 2\rceil} w_{j}^{*} \leq 2 \sum_{j=1}^{k^{*}} w_{j}^{*} \leq 2 O P T$, since $k^{*} \geq\left\lceil\frac{k}{2}\right\rceil$. A tight example for this algorithm, is given in Section 5.2, as for large values of $b$ the $\operatorname{EC}(w, b)$ coincides with the $\operatorname{EC}(w)$ problem. By a careful analysis, the complexity of both Lines 2-5 and 7-9 of the algorithm is $O(|V| \log |V|)$. 


\section{Chapter 7}

\section{Bounded Max-Vertex-Coloring}

In this chapter we first present a simple 2-approximation algorithm for the $\mathrm{VC}(w, b)$ problem on bipartite graphs. The unweighted variant of this algorithm gives a $\frac{4}{3}$ approximation ratio for the $\mathrm{VC}(b)$ problem on bipartite graphs, which closes the approximability question for this case. Then, we give a generic scheme which becomes a $\frac{17}{11}$-approximation algorithm for bipartite graphs, a PTAS for bipartite graphs and fixed $b$, as well as a PTAS for trees. Recall also that by Theorem 3 there is an $H_{b}$ approximation ratio for general graphs, if $b$ is fixed.

\subsection{A simple split algorithm}

Let $G=(U \cup V, E),|U \cup V|=n$, be a vertex weighted bipartite graph. Our first algorithm colors the vertices of each class of $G$ separately, by finding the ordered $b$-partitions of classes $U$ and $V$. For the minimum number of colors $k^{*}$ it holds that $k^{*} \geq\left\lceil\frac{|U|+|V|}{b}\right\rceil$ and, therefore, $k=\left\lceil\frac{|U|}{b}\right\rceil+\left\lceil\frac{|V|}{b}\right\rceil \leq\left\lceil\frac{|U|+|V|}{b}\right\rceil+1 \leq k^{*}+1$.

\section{Algorithm Split}

1: Let $\mathcal{P}_{U}=\left\{U_{1}, U_{2}, \ldots, U_{k_{U}}\right\}$ be the ordered $b$-partition of $U$;

2: Let $\mathcal{P}_{V}=\left\{V_{1}, V_{2}, \ldots, V_{k_{V}}\right\}$ be the ordered $b$-partition of $V$;

3: Return the coloring $\mathcal{C}=\mathcal{P}_{U} \cup \mathcal{P}_{V}$;

Theorem 22. Algorithm Split returns a solution of weight $W \leq 2 \cdot w_{1}^{*}+w_{2}^{*}+$ $\ldots+w_{k^{*}}^{*} \leq 2 \cdot O P T$ for the $\mathrm{VC}(w, b)$ problem in bipartite graphs.

Proof. Let $\langle\mathcal{C}\rangle=\left\langle C_{1}, C_{2}, \ldots, C_{k}\right\rangle$ be the colors constructed by Algorithm Split, that is $w_{1} \geq w_{2} \geq \ldots \geq w_{k}$. Assume, w.l.o.g., that $U_{x}, 1 \leq x \leq k_{U}$, is the $i-t h$ color in $\langle\mathcal{C}\rangle$. Let also $u$ be the heaviest vertex of $U_{x}$, that is $w(u)=w_{i}$.

The ordered $b$-partition of $U$ and $V$ implies that the colors that appear before $U_{x}$ in $\langle\mathcal{C}\rangle$ are the colors $U_{1}, U_{2}, \ldots, U_{x-1}$ and $V_{1}, V_{2}, \ldots, V_{y}, y=i-x$. The colors $U_{1}, U_{2}, \ldots, U_{x-1}$ are all of cardinality $b$ and their $(x-1) \cdot b$ vertices are all of weight at least $w(v)$. The colors $V_{1}, V_{2}, \ldots, V_{y-1}$, are also all of cardinality $b$ and their $(y-1) \cdot b$ vertices are all of weight at least the weight of the heaviest vertex of color $V_{y}$ which 
is at least $w(v)$. Taking into account the vertex $v$ itself it follows that there are in $G$ at least $(x-1) \cdot b+[(y-1) \cdot b+1]+1=(x+y-2) \cdot b+2=(i-2) \cdot b+2$ vertices of weight at least $w(v)=w_{i}$. In an optimal solution, these vertices belong into at least $\left\lceil\frac{(i-2) \cdot b+2}{b}\right\rceil=(i-1)$ colors, each one of weight at least $w_{i}$. Hence, $w_{i-1}^{*} \geq w_{i}$, $2 \leq i \leq k$. Clearly, $w_{1}=w_{1}^{*}$, since both are equal to the weight of the heaviest vertex of the graph, and as $k \leq k^{*}+1$, we obtain

$$
\begin{aligned}
W & =\sum_{i=1}^{k} w_{i}=w_{1}^{*}+\sum_{i=2}^{k} w_{i} \leq w_{1}^{*}+\sum_{i=1}^{k-1} w_{i}^{*} \\
& \leq w_{1}^{*}+\sum_{i=1}^{k^{*}} w_{i}^{*}=2 \cdot w_{1}^{*}+w_{2}^{*}+\ldots+w_{k^{*}}^{*} \leq 2 \cdot O P T .
\end{aligned}
$$

The complexity of Algorithm Split is dominated by the sorting needed to obtain the ordered $b$-partitions of $U$ and $V$ in Lines 1 and 2, that is $O(|V| \cdot \log |V|)$.

Algorithm Split applies also to the VC $(b)$ problem on bipartite graphs. Moreover, the absence of weights in the $\mathrm{VC}(b)$ problem allows a tight analysis with respect to the $\frac{4}{3}$ inapproximability bound.

Theorem 23. There is a $\frac{4}{3}$-approximation algorithm for the $\mathrm{VC}(b)$ problem on bipartite graphs.

Proof. Assume, first, that $|U|+|V| \geq 2 b+1$. Then, $k^{*} \geq\left\lceil\frac{2 b+1}{b}\right\rceil=3$ and, since $k \leq k^{*}+1$, we get $\frac{k}{k^{*}} \leq \frac{4}{3}$.

Assume, next, that $b<|U|+|V| \leq 2 b$. In this case the optimal solution consists of two or three colors and it is polynomial to decide between them. In fact, by Theorem 1(ii), it is polynomial to decide if a bipartite graph can be colored with two colors even for the general $\mathrm{VC}\left(\phi, b_{i}\right)$ problem.

Assume, finally, that $|U|+|V| \leq b$. Then, an optimal solution consists of either two colors (if $E \neq \emptyset$ ) or one color (if $E=\emptyset$ ).

\subsection{A generic scheme}

In this section we combine the ideas used in Section 5.4 for the $\mathrm{EC}(w)$ problem with Algorithm Split to design a generic scheme for the $\operatorname{VC}(w, b)$ problem.

To obtain our scheme we split a bipartite graph $G=(U \cup V, E),|U \cup V|=n$, into two subgraphs $G_{1, j}$ and $G_{j+1, n}$ induced by the $j$ heaviest and the $n-j$ lightest vertices of $G$, respectively (by convention, we consider $G_{1,0}$ as an empty subgraph). Our scheme depends on a parameter $p$ such that all the vertices of $G$ of weights $w_{1}^{*}, w_{2}^{*}, \ldots, w_{p-1}^{*}$ are in a subgraph $G_{1, j}$. This is always possible for some $j \leq b(p-1)$, since each color of an optimal solution for $G$ contains at most $b$ vertices. In fact, for every $j, 1 \leq j \leq b(p-1)$, we obtain a solution for the whole graph by concatenating 
an optimal solution of at most $p-1$ colors for $G_{1, j}$, if there is one, and the solution obtained by Algorithm Split for $G_{j+1, n}$.

\section{Algorithm Scheme $(p)$}

1: Let $\langle U \cup V\rangle=\left\langle u_{1}, u_{2}, \ldots u_{n}\right\rangle$;

2: for $j=0$ to $b \cdot(p-1)$ do

3: $\quad$ Split the graph into two vertex induced subgraphs:

- $G_{1, j}$ induced by vertices $u_{1}, u_{2}, \ldots, u_{j}$

- $G_{j+1, n}$ induced by vertices $u_{j+1}, u_{j+2}, \ldots, u_{n}$

4: $\quad$ if there is a solution for $G_{1, j}$ with at most $p-1$ colors then

5: $\quad$ Find an optimal solution for $G_{1, j}$ with at most $p-1$ colors;

6: $\quad$ Run Algorithm Split for $G_{j+1, n}$;

7: $\quad$ Concatenate the two solutions found in Lines 5 and 6;

8: $\quad$ end if

9: end for

10: Return the best solution found;

Lemma 6. Algorithm $\operatorname{SCheme}(p)$ achieves a $\left(1+\frac{1}{H_{p}}\right)$ approximation ratio for the $\operatorname{VC}(w, b)$ problem.

Proof. Consider the iteration $j, j \leq b \cdot(p-1)$, of the algorithm where the weight of the heaviest vertex in $G_{j+1, n}$ equals to the weight of the $i$-th color of an optimal solution, i.e. $w\left(u_{j+1}\right)=w_{i}^{*}, 1 \leq i \leq p$.

The vertices of $G_{1, j}$ are a subset of those appeared in the $i-1$ heaviest colors of the optimal solution. Thus, an optimal solution for $G_{1, j}$ is of weight

$$
O P T_{1, j} \leq w_{1}^{*}+w_{2}^{*}+\ldots+w_{i-1}^{*} .
$$

The vertices of $G_{j+1, n}$ are a superset of those appeared in the $k^{*}-(i-1)$ lightest colors of the optimal solution. The extra vertices of $G_{j+1, n}$ are of weight at most $w_{i}^{*}$ and appear in an optimal solution into at most $i-1$ colors. Thus, an optimal solution for $G_{j+1, n}$ is of weight $O P T_{j+1, n} \leq w_{i}^{*}+w_{i+1}^{*}+\ldots+w_{k^{*}}^{*}+(i-1) \cdot w_{i}^{*}=$ $i \cdot w_{i}^{*}+w_{i+1}^{*}+\ldots+w_{k^{*}}^{*}$. By Theorem 22, Algorithm Split returns a solution for $G_{j+1, n}$ of weight

$$
W_{j+1, n} \leq(i+1) \cdot w_{i}^{*}+w_{i+1}^{*}+\ldots+w_{k^{*}}^{*}
$$

Therefore, the solution found in this iteration $j$ for the whole graph $G$ is of weight

$$
W_{i}=O P T_{1, j}+W_{j+1, n} \leq w_{1}^{*}+w_{2}^{*}+\ldots+w_{i-1}^{*}+(i+1) \cdot w_{i}^{*}+w_{i+1}^{*}+\ldots+w_{k^{*}}^{*} .
$$

In all the iterations of the algorithm we obtain $p$ such inequalities for $W$. By multiplying the $i$-th, $1 \leq i \leq p$, inequality by $\frac{1}{i \cdot\left(H_{p}+1\right)}$ and adding up all of them, we have $\left(\sum_{i=1}^{p} \frac{1}{i \cdot\left(H_{p}+1\right)}\right) \cdot W \leq O P T$, that is $\frac{W}{O P T} \leq \frac{H_{p}+1}{H_{p}}=1+\frac{1}{H_{p}}$. 
The complexity of the Algorithm $\operatorname{Scheme}(p)$ is $O(b p(f(p)+|V| \log |V|))$, where $O(f(p))$ is the complexity of checking for the existence of solutions with at most $p-1$ colors for $G_{1, j}$ and finding an optimal one among them, while $O(|V| \log |V|)$ is the complexity of Algorithm Split. Algorithm Scheme(1) coincides with AlgoRithm Split. Algorithm Scheme(2) has simply to check if the $j \leq b$ vertices of $G_{1, j}$ are independent from each other and, therefore, it derives a $\frac{5}{3}$ approximate solution in polynomial time. Algorithm Scheme(3) has to check and find, a two color solution for $G_{1, j}$, if any. This can be done in polynomial time by Theorem 2(ii). Thus, Algorithm Scheme(3) is a polynomial time $\frac{17}{11}$-approximation algorithm for the $\operatorname{VC}(w, b)$ problem on bipartite graphs.

However, when $p \geq 4$ and $b$ is a part of the instance, finding an optimal solution in $G_{1, j}$ is an NP-hard problem (even for the $\mathrm{VC}(b)$ problem [6]). Hence, we consider that $b$ is a fixed constant. In this case, we run an exhaustive algorithm for finding, if any, an optimal solution in $G_{1, j}$ of at most $p-1$ colors. The complexity of such an exhaustive algorithm is $O\left((p-1)^{b \cdot(p-1)}\right)$ and thus, the complexity of AlGORITHM $\operatorname{Scheme}(p), p \geq 4$, becomes $O\left(b p^{b p}+n^{2} \log n\right)$, since $b p$ is $O(|V|)$. Choosing $p$ such that $\epsilon=\frac{1}{H_{p}}$, we get $p=O\left(2^{\frac{1}{\epsilon}}\right)$. Consequently, for fixed $b$, we have a PTAS for the $\mathrm{VC}(w, b)$ problem on bipartite graphs, that is an approximation ratio of $1+\frac{1}{H_{p}}=1+\epsilon$ within $O\left(b\left(2^{\frac{1}{\epsilon}}\right)^{b 2^{\frac{1}{\epsilon}}}+|V|^{2} \log |V|\right)$ time.

Furthermore, in the particular case of trees, checking the existence of solutions with at most $p-1$ colors for $G_{1, j}$, and finding an optimal one among them, can be done, by Theorem 2(i), in polynomial time for fixed $p$. The complexity of our scheme in this case becomes $O\left(b 2^{\frac{1}{\epsilon}}\left(|V|^{2^{\frac{1}{\epsilon}}}+|V|^{2} \log |V|\right)\right)$. Therefore, the following theorem holds.

Theorem 24. For the $\operatorname{VC}(w, b)$ problem, Algorithm $\operatorname{Scheme}(p)$ is a (i) polynomial time $\frac{17}{11}$-approximation algorithm for bipartite graphs (for $p=3$ ),

(ii) PTAS for bipartite graphs if b is fixed,

(iii) PTAS for trees. 


\section{Chapter 8}

\section{Conclusions and open questions}

We presented complexity results and approximation algorithms for (bounded) maxcoloring problems that arise in computer and communication systems, with respect to the class of the underlying graph. Our results concern general and bipartite graphs, trees and bi-valued graphs.

For the $\mathrm{EC}(w)$ problem, recall that it is known to be approximable within a factor of 2 (for any class of graphs) and inapproximable within a factor less than $7 / 6$ (even for bipartite graphs), while its complexity for trees remains open. We narrow these gaps in the approximability of the problem by presenting an 1.74approximation algorithm for bipartite graphs, and a PTAS for trees. Moreover, we prove that the problem is NP-complete for complete bi-valued graphs and we present an asymptotic $4 / 3$-approximation algorithm for general bi-valued graphs.

The $\operatorname{EC}(w, b)$ problem is NP-complete for bipartite graphs as a generalization of the $\mathrm{EC}(w)$ problem. We prove that it is NP-complete even on trees, which is the first complexity result for all the (bounded) max-coloring problems on this class of graphs. On the other hand, we present approximation algorithms of ratio at most 3 for general and bipartite graphs and 2 for trees.

The $\mathrm{VC}(w, b)$ problem is non approximable by a constant factor approximation ratio on general graphs, as a generalization of the classical vertex coloring problem, as well as by a factor within $4 / 3$ on bipartite graphs, a result that comes from the $\mathrm{VC}(b)$ problem. We present approximation algorithms of ratio $\left\lceil\frac{b}{2}\right\rceil$ for general graphs and 17/11 for bipartite graphs, and a PTAS for trees. Finally, for the $\mathrm{VC}(b)$ problem, we show that the known $4 / 3$ lower bound for bipartite graphs is tight by providing a 4/3-approximation algorithm.

The recent research activity on the (bounded) max-coloring problems has led to a significant progress on understanding their complexity and approximability, though there is enough room for further research. A first category of open questions can be picked up by looking the entries of Table 1.1.

i. Further identification of the frontier between polynomial and NP-hard variants of the problems with respect to the underlying graph. For example, the complexity of all the $\operatorname{VC}(w), \operatorname{VC}(w, b)$ and $\mathrm{EC}(w)$ problems on trees remains the most interesting open question. 
ii. Decrease (or close) the gaps between the best known approximation ratios and inapproximability bounds, either by obtaining new improved approximation algorithms, or by strengthening existing inapproximability results. For example, a 2-approximation algorithm and a 4/3-inapproximability result are known for the $\operatorname{EC}(w)$ problem on general graphs of any maximum degree. The gap is narrower for bipartite graphs, as we presented an 1.74-approximation algorithm and a 7/6-inapproximability result is known. Moreover, the NP-completeness proof for the $\operatorname{EC}(w, b)$ problem on trees does not prevent the existence of a better ratio than the 2 approximation one we presented, or even a PTAS, such as for the $\operatorname{VC}(w), \operatorname{VC}(w, b)$ and $\operatorname{EC}(w)$ problems. Hence, it would be interesting to decrease these approximability gaps by improving either the lower or the upper bounds.

A second category of interesting questions is related to the nature of the applications of the (bounded) max-coloring problems:

i. As we have already mentioned in Chapter 1 all the (bounded) max-coloring problems are equivalent to the parallel batch scheduling problem with incompatibilities between jobs. Within this context, a natural idea to decrease the weight of a solution to such a weighted coloring problem is to allow preemption, that is interrupt the execution of a job and complete it later. It is known that the preemptive $\mathrm{EC}(w)$ problem for bipartite graphs is equivalent to the preemptive open shop scheduling problem which can be solved optimally in polynomial time [48]. An interesting question here is the complexity of the preemptive $\operatorname{EC}(w)$ problem on general graphs. Similar questions are also of interest for the $\mathrm{VC}(w)$ problem.

ii. In the same scheduling context, there a significant setup delay, say $d$, to establish each batch. For the (bounded) max-coloring problems the presence of such a delay can be easily handled by increasing the weight of the vertices or edges of $G$ by $d$. This way the weight of each batch will be also increased by $d$, incorporating its set-up delay. However, for the preemptive variants of the problems the existence of the setup delay plays a crucial role, since the preemption increases the number of batches as well as the total setup overhead.

iii. The applications of all problems are on-line in nature. In practice, buffers are allocated as memory requests are created, and messages are scheduled as they arrive, with out knowledge of future. The online version of both problems is therefore of great practical and theoretical interest.

Finally, a third category of open questions includes some problems of technical nature.

i. The 1.74 approximation ratio of the algorithm we presented for the $\mathrm{EC}(w)$ problem on bipartite graphs, is obtained using computational tools (Mathematica). It would be interesting to have a close formula for this ratio. 
ii. Our analysis of the approximation algorithm for the $\mathrm{EC}(w, b)$ problem presented, yields ratios of $3-\frac{2}{\sqrt{2 b}}$ for general graphs and $3-\frac{2}{\sqrt{b}}$ for bipartite graphs. However, this algorithm reduces to the greedy 2-approximation one for the $\operatorname{EC}(w)$ problem when the cardinality bound $b$ is large enough. Hence, the ratios for the $\operatorname{EC}(w, b)$ problem should tend to 2 instead of increasing with the bound $b$. Thus, a question here is a better analysis of our algorithm to match this fact. 


\section{Bibliography}

[1] F. N. Afrati, T. Aslanidis, E. Bampis, and I. Milis. Scheduling in switching networks with set-up delays. Journal of Combinatorial Optimization, 9:49-57, 2005 .

[2] N. Alon. A note on the decomposition of graphs into isomorphic matchings. Acta Mathematica Hungarica, 42:221-223, 1983.

[3] R. P. Anstee. An algorithmic proof of Tutte's $f$-factor theorem. Journal of Algorithms, 6:112-131, 1985.

[4] B. S. Baker and E. G. Coffman Jr. Mutual exclusion scheduling. Theoretical Computer Science, 162:225-243, 1996.

[5] A. Björklund and Th. Husfeldt. Inclusion-exclusion algorithms for counting set partitions. In 47th Annual IEEE Symposium on Foundations of Computer Science (FOCS'06), pages 575-582. IEEE Computer Society, 2006.

[6] H. L. Bodlaender and K. Jansen. Restrictions of graph partition problems. Part I. Theoretical Computer Science, 148:93-109, 1995.

[7] V. A. Bojarshinov. Edge and total coloring of interval graphs. Discrete Applied Mathematics, 114:23-28, 2001.

[8] G. Bongiovanni, D. Coppersmith, and C. K. Wong. An optimum time slot assignment algorithm for an SS/TDMA system with variable number of transponders. IEEE Trans. on Communications, 29:721-726, 1981.

[9] M. Boudhar. Scheduling on a batch processing machine with split compatibility graphs. Journal of Mathematical Modelling and Algorithms, 4:391-407, 2005.

[10] M. Boudhar and G. Finke. Scheduling on a batch machine with job compatibilities. Belgian Journal of Oper. Res., Statistics and Computer Science, 40:69-80, 2000 .

[11] L. Cai and J. A. Ellis. Np-completeness of edge-coloring some restricted graphs. Discrete Applied Mathematics, 30:15-27, 1991.

[12] B.-L. Chen, H.-L. Fu, and M. T. Ko. Total chromatic number and chromatic index of split graphs. Journal of Combinatorial Mathematics and Combinatorial Computing, 17:137•-146, 1995. 
[13] A. G. Chetwynd and A. J. W. Hilton. Regular graphs of high degree are 1factorizable. In Proceedings of the London Mathematical Society, volume 50, pages 193-206, 1985.

[14] V. Chvátal. A greedy heuristic for the set-covering problem. Mathematics of Operations Research, 4:233-235, 1979.

[15] J. Cohen, E. Jeannot, N. Padoy, and F. Wagner. Messages scheduling for parallel data redistribution between clusters. IEEE Transactions on Parallel and Distributed Systems, 17:1163-1175, 2006.

[16] R. Cole, K. Ost, and S. Schirra. Edge-coloring bipartite multigraphs in $O(E \log D)$ time. Combinatorica, 21:5-12, 2001.

[17] P. Crescenzi, X. Deng, and Ch. H. Papadimitriou. On approximating a scheduling problem. Journal of Combinatorial Optimization, 5:287-297, 2001.

[18] D. de Werra. Decomposition of bipartite multigraphs into matchings. Mathematical Methods of Operations Research, 16:85-90, 1972.

[19] D. de Werra. Restricted coloring models for timetabling. Discrete Mathematics, 165/166:161-170, 1997.

[20] D. de Werra, M. Demange, B. Escoffier, J. Monnot, and V. Th. Paschos. Weighted coloring on planar, bipartite and split graphs: Complexity and approximation. Discrete Applied Mathematics, 157:819-832, 2009.

[21] D. de Werra, A. Hertz, D. Kobler, and N. V. R. Mahadev. Feasible edge coloring of trees with cardinality constraints. Discrete Mathematics, 222:61-72, 2000.

[22] M. Demange, D. de Werra, J. Monnot, and V. Th. Paschos. Time slot scheduling of compatible jobs. Journal of Scheduling, 10:111-127, 2007.

[23] M. Dror, G. Finke, S. Gravier, and W. Kubiak. On the complexity of a restricted list-coloring problem. Discrete Mathematics, 195:103-109, 1999.

[24] L. Epstein and A. Levin. On the max coloring problem. In 5th Workshop on Approximation and Online Algorithms (WAOA'07), volume 4927 of LNCS, pages 142-155. Springer, 2008.

[25] B. Escoffier, J. Monnot, and V. Th. Paschos. Weighted coloring: Further complexity and approximability results. Information Processing Letters, 97:98$103,2006$.

[26] G. Finke, V. Jost, M. Queyranne, and A. Sebő. Batch processing with interval graph compatibilities between tasks. Discrete Applied Mathematics, 156:556$568,2008$.

[27] S. Fiorini and R. J. Wilson. Edge-colourings of graphs. Pitman, London, 1977. 
[28] S. Fiorini and R. J. Wilson. Edge colorings of graphs. In L. W. Beineke and R. J. Wilson, editors, Selected Topics in Graph Theory, pages 103-126. Academic Press, 1978.

[29] H. N. Gabow, T. Nishizeki, O. Kariv, D. Leven, and O. Terada. Algorithms for edge-coloring graphs. Technical Report TRECIS-8501, Tohoku University, 1985 .

[30] F. Gardi. Mutual exclusion scheduling with interval graphs or related classes. Part II. Discrete Applied Mathematics, 156:794-812, 2008.

[31] M. R. Garey, D. S. Johnson, G. L. Miller, and C. H. Papadimitriou. The complexity of coloring circular arcs and chords. SIAM Journal on Algebraic and Discrete Methods, 1:216-227, 1980.

[32] M. R. Garey, D. S. Johnson, and L. J. Stockmeyer. Some simplified NP-complete graph problems. Theoretical Computer Science, 1:237-267, 1976.

[33] F. Gavril. Algorithms for minimum coloring, maximum clique, minimum covering by cliques, and maximum independent set of a chordal graph. SIAM Journal on Computing, 1:180-187, 1972.

[34] M. C. Golumbic. Algorithmic graph theory and perfect graphs. Academic Press, New York, 1980.

[35] I. S. Gopal and C. Wong. Minimizing the number of switchings in a SS/TDMA system. IEEE Transactions On Communications, 33:497-501, 1985.

[36] S. Gravier, D. Kobler, and W. Kubiak. Complexity of list coloring problems with a fixed total number of colors. Discrete Applied Mathematics, 117:65-79, 2002 .

[37] M. Grötschel, L. Lovász, and A. Schrijver. The ellipsoid method and its consequences in combinatorial optimization. Combinatorica, 1:169-197, 1981.

[38] M. M. Halldórsson. A still better performance guarantee for approximate graph coloring. Information Processing Letters, 45:19-23, 1993.

[39] M. M. Halldórsson and H. Shachnai. Batch coloring flat graphs and thin. In 11th Scandinavian Workshop on Algorithm Theory (SWAT'08), volume 5124 of LNCS, pages 198-209. Springer, 2008.

[40] P. Hansen, A. Hertz, and J. Kuplinsky. Bounded vertex colorings of graphs. Discrete Mathematics, 111:305-312, 1993.

[41] I. Holyer. The NP-completeness of edge-coloring. SIAM Journal on Computing, 10:718-720, 1981.

[42] M. Jarvis and B. Zhou. Bounded vertex coloring of trees. Discrete Mathematics, 232:145-151, 2001. 
[43] D. S. Johnson. The np-completeness column: An ongoing guide. Journal of Algorithms, 6:434-451, 1985.

[44] I. A. Karapetian. On coloring of arc graphs. Akademiia nauk Armianskoi SSR Doklady, 70:306•-311, 1980.

[45] R. M. Karp. Reducibility among combinatorial problems. In R. E. Miller and J. W. Thatcher, editors, Complexity of Computer Computations, pages 85•-103. Plenum Press, 1972.

[46] A. Kesselman and K. Kogan. Nonpreemptive scheduling of optical switches. IEEE Trans. on Communications, 55:1212-1219, 2007.

[47] D. König. Über graphen und ihre anwendung auf determinantentheorie und mengenlehre. Mathematische Annalen, 77:453-465, 1916.

[48] E. L. Lawler and J. Labetoulle. On preemptive scheduling of unrelated parallel processors by linear programming. Journal of the Association for Computing Machinery, 25:612-619, 1978.

[49] R. C. S. Machado, C. M. H. de Figueiredo, and K. Vušković. Chromatic index of graphs with no cycle with a unique chord. preprint, 2009.

[50] S. Micali and V. V. Vazirani. An $O(\sqrt{|V|}|E|)$ algorithm for finding maximum matching in general graphs. In 21st Annual IEEE Symposium on Foundations of Computer Science (FOCS'80), pages 17-27. IEEE Computer Society, 1980.

[51] S. V. Pemmaraju and R. Raman. Approximation algorithms for the maxcoloring problem. In 32nd International Colloquium on Automata, Languages and Programming (ICALP'05), volume 3580 of LNCS, pages 1064-1075. Springer, 2005.

[52] S. V. Pemmaraju, R. Raman, and K. R. Varadarajan. Buffer minimization using max-coloring. In 15th Annual ACM-SIAM Symposium on Discrete Algorithms (SODA'04), pages 562-571, 2004.

[53] F. Rendl. On the complexity of decomposing matrices arising in satellite communication. Operations Research Letters, 4:5-8, 1985.

[54] N. Robertson, D. P. Sanders, P. D. Seymour, and R. Thomas. The four colour theorem. Journal of Combinatorial Theory, Series B, 70:2-44, 1997.

[55] D. P. Sanders and Y. Zhao. Planar graphs of maximum degree seven are Class I. Journal of Combinatorial Theory, Series B, 83:201-212, 2001.

[56] V. G. Vizing. On an estimate of the chromatic class of a p-graph. Diskret. Analiz, 3:25-30, 1964.

[57] V. G. Vizing. Critical graphs with given chromatic index. Diskret. Analiz, 5:9-17, 1965. 
[58] D. Zuckerman. Linear degree extractors and the inapproximability of max clique and chromatic number. In 38th Annual ACM Symposium on the Theory of Computing (STOC'06), pages 681-690, 2006. 